\title{
Classification of degenerations and Picard lattices of Kählerian K3 surfaces with the symplectic automorphism group $\left(C_{2}\right)^{2}$.
}

\author{
Viacheslav V. Nikulin
}

7 March 2021

\begin{abstract}
In our papers of 2013 - 2018, we classified degenerations and Picard lattices of Kählerian K3 surfaces with finite symplectic automorphism groups of high order. For remaining groups of small order: $D_{6}, C_{4},\left(C_{2}\right)^{2}, C_{3}, C_{2}$ and $C_{1}$, it was not completely considered because each of these cases requires very long and difficult considerations and calculations. Cases of $D_{6}$ and $C_{4}$ were recently completely considered in [19] and [20].

Here we consider the analogous complete classification for the group $\left(C_{2}\right)^{2}$ of the order 4 .
\end{abstract}

Dedicated to the memory of Vitaliy Sergeevich Makarov

\section{Introduction}

In our papers [13] - 18, we considered classification of degenerations and Picard lattices of Kählerian K3 surfaces with finite symplectic automorphism groups. In [13] - [18], we completed this classification for the groups of high order. For remaining groups of small orders: $D_{6}, C_{4},\left(C_{2}\right)^{2}, C_{3}, C_{2}$ and $C_{1}$, the complete classification was not considered in these papers. Here $D_{n}$ is the dihedral group, and $C_{n}$ is the cyclic group of order $n$. For these groups, there are too many cases to consider, and it is better to consider each of these groups separately. The cases of $D_{6}$ and $C_{4}$ were recently completely considered in [19], [20].

In this paper, we consider similar complete classification for the group $\left(C_{2}^{2}\right)$ of the order 4 . 
This classification is given in Tables 1, 2 and 3 of Section 2 .

We use the same notations, methods and results as in our papers [13] [19]. See more details in Section 2 .

A preliminary variant of this paper was published in preprint [20].

\section{Classification of degenerations and Picard lattices $S$ of Kählerian K3 surfaces with finite symplectic automorphism groups. General theory and the case of the group $\left(C_{2}\right)^{2}$.}

Here we remind to a reader our methods and results from [13] - [19] for classification of degenerations and Picard lattices of Kählerian K3 surfaces with finite symplectic automorphism groups. Here we apply them to the group $\left(C_{2}\right)^{2}$ of the order 4 which was not completely considered in these papers.

Let $X$ be a Kählerian K3 surface (e. g. see [23], 22], 2], 24], 25] about such surfaces). That is $X$ is a non-singular compact complex surface with the trivial canonical class $K_{X}$, and with the irregularity $q(X)=0$. It is Kählerian by [24] and [25]. Then $H^{2}(X, \mathbb{Z})$ with the intersection pairing is an even unimodular lattice $L_{K 3}$ of the signature $(3,19)$. Further, a lattice means a non-degenerate integer symmetric bilinear form of a finite rank. For a non-zero holomorphic 2-form $\omega_{X} \in \Omega^{2}[X]$, we have $H^{2,0}(X)=\Omega^{2}[X]=\mathbb{C} \omega_{X}$. The primitive sublattice

$$
S_{X}=H^{2}(X, \mathbb{Z}) \cap H^{1,1}(X)=\left\{x \in H^{2}(X, \mathbb{Z}) \mid x \cdot H^{2,0}(X)=0\right\} \subset H^{2}(X, \mathbb{Z})
$$

is the Picard lattice of $X$ generated by first Chern classes of all line bundles over $X$. We remind that a primitive sublattice means that $H^{2}(X, \mathbb{Z}) / S_{X}$ has no torsion.

Let $G$ be a finite symplectic automorphism group of a K3 surface $X$. See [10], [11], [12], 8], 6], [26], [5] about their classification. Here symplectic means that for any $g \in G$, for a non-zero holomorphic 2-form $\omega_{X} \in H^{2,0}(X)=\Omega^{2}[X]=\mathbb{C} \omega_{X}$, one has $g^{*}\left(\omega_{X}\right)=\omega_{X}$. For an $G$-invariant sublattice $M \subset H^{2}(X, \mathbb{Z})$, we denote by $M^{G}=\{x \in M \mid G(x)=x\}$ the fixed sublattice of $M$, and by $M_{G}=\left(M^{G}\right)_{M}^{\perp}$ the coinvariant sublattice of $M$. By [10], [11], the coinvariant lattice $S_{G}=H^{2}(X, \mathbb{Z})_{G}=\left(S_{X}\right)_{G}$ is Leech type lattice: it is negative definite, it has no elements with square $(-2), G$ acts trivially on the discriminant group $A_{S_{G}}=\left(S_{G}\right)^{*} / S_{G}$, and $\left(S_{G}\right)^{G}=\{0\}$. For a general pair $(X, G)$, the $S_{G}=S_{X}$, and nongeneral $(X, G)$ can be considered as Kählerian K3 surfaces with the condition $S_{G} \subset S_{X}$ on the Picard lattice (in terminology of [11]). The dimension of their moduli is equal to $20-\operatorname{rk} S_{G}$. 
Let $E \subset X$ be a non-singular irreducible rational curve (that is $E \cong \mathbb{P}^{1}$ ). It is equivalent to: $\alpha=\operatorname{cl}(E) \in S_{X}, \alpha^{2}=-2, \alpha$ is effective and $\alpha$ is numerically effective: $\alpha \cdot D \geq 0$ for any irreducible curve $D$ on $X$ such that $\operatorname{cl}(D) \neq \alpha$.

Let us consider $t$ non-singular irreducible rational curves $E_{1}, \ldots, E_{t}$ on $X$ with classes $\alpha_{i}=\operatorname{cl}\left(E_{i}\right) \in S_{X}$ such that their orbits $G\left(E_{1}\right), \ldots, G\left(E_{t}\right)$ are different. Let us consider the primitive sublattice

$$
S=\left[S_{G}, G\left(\alpha_{1}\right), \ldots, G\left(\alpha_{t}\right)\right]_{p r}=\left[S_{G}, \alpha_{1}, \ldots, \alpha_{t}\right]_{p r} \subset S_{X}
$$

of $S_{X}$ generated by the coinvariant sublattice $S_{G}$ and all classes of the orbits $G\left(E_{1}\right), \ldots G\left(E_{t}\right)$ and assume that $S$ is negative definite. Since $S_{G}$ has no elements with square $(-2)$, it follows that rk $S=\operatorname{rk} S_{G}+t$ and elements of orbits $G\left(\alpha_{1}\right), \ldots G\left(\alpha_{t}\right)$ define the basis of the root system $\Delta(S)$ of all elements with square $(-2)$ of $S$. All curves $G\left(E_{1}\right), \ldots, G\left(E_{t}\right)$ of $X$ can be contracted to Du Val singularities of types of connected components of the Dynkin diagram of the basis. The group $G$ will act on the corresponding singular K3 surface $\bar{X}$ with these Du Val singularities. For a general such collection $\left(X, G, G\left(E_{1}\right), \ldots G\left(E_{t}\right)\right)$, the Picard lattice $S_{X}=S$, and such collection can be considered as a degeneration of codimension $t$ of Kählerian K3 surfaces $(X, G)$ with the finite symplectic automorphism group $G$. Really, the dimension of moduli of Kählerian K3 surfaces with the condition $S \subset S_{X}$ on the Picard lattice is equal to $20-\mathrm{rk} S=20-\mathrm{rk} S_{G}-t$.

We can consider only maximal finite symplectic automorphism groups $G$ with the same coinvariant lattices $S_{G}$, that is $G=C \operatorname{los}(G)$. By Global Torelli Theorem for K3 surfaces [22], [2], this is equivalent to

$$
G \mid S_{G}=\left\{g \in O\left(S_{G}\right) \mid g \text { is identity on } A_{S_{G}}=\left(S_{G}\right)^{*} / S_{G}\right\} .
$$

The type of the degeneration is given by the Dynkin diagrams and subdiagrams

$$
\left(\operatorname{Dyn}\left(G\left(\alpha_{1}\right)\right), \ldots, \operatorname{Dyn}\left(G\left(\alpha_{t}\right)\right)\right) \subset \operatorname{Dyn}\left(G\left(\alpha_{1}\right) \cup \cdots \cup G\left(\alpha_{t}\right)\right)
$$

and their types. Numberings of subdiagrams $\operatorname{Dyn}\left(G\left(\alpha_{i}\right)\right)$ and connected components of $\operatorname{Dyn}\left(G\left(\alpha_{1}\right) \cup \cdots \cup G\left(\alpha_{t}\right)\right)$ must agree. In difficult cases, we also consider the matrix of subdiagrams which is defined by

$$
\left(\operatorname{Dyn}\left(G\left(\alpha_{i}\right)\right), \operatorname{Dyn}\left(G\left(\alpha_{j}\right)\right)\right) \subset \operatorname{Dyn}\left(G\left(\alpha_{i}\right) \cup G\left(\alpha_{j}\right)\right)
$$

and their types for $1 \leq i<j \leq t$.

By Global Torelli Theorem for K3 surfaces [22], [2], the type of the abstract group $G=\operatorname{Clos}(G)$ which is equivalent to the isomorphism class of the coinvariant lattice $S_{G}$, and the type of the degeneration give the main invariants of the degeneration. 
For groups $G=\operatorname{Clos}(G)$ of order $|G|>4$ and $G=C_{4}$ classification of degenerations of arbitrary codimension and Picard lattices $S$ is given in tables of [15] [19]. In this paper, applying the same results and methods, we give similar classification of degenerations of arbitrary codimension and Picard lattices $S$ for the group $G=\left(C_{2}\right)^{2}$. It is given in Table 1 where we give all possible types of degenerations in the column Deg. We use the standard notations $\mathbb{A}_{n}, \mathbb{D}_{n}, \mathbb{E}_{n}$ for connected components of Dynkin diagrams with $n$ vertices (see [1]), and $A_{n}, D_{n}, E_{n}$ the corresponding root lattices. By $\Gamma_{1} \amalg \Gamma_{2}$ we denote the disjoint union of diagrams $\Gamma_{1}$ and $\Gamma_{2}$ and by $\oplus$ the orthogonal sum of lattices. By $k M$ we denote the orthogonal sum of $k$ copies of a lattice $M$. For a given degeneration Deg, at the same line, using results of [12], we give the genus of the lattices $S$ by rk $S$ and the discriminant quadratic form $q_{S}$ in notations of [3]. By $*$, we denote cases when we also prove that the lattice $S$ is unique up to isomorphisms for the given type.

In Table 2, we give some markings by Niemeier lattices $N_{j}, j=1, \ldots, 24$, of lattices $S$ of a degeneration Deg together with the action of $G$ on $S$ and its orbits $G\left(\alpha_{1}\right), \ldots G\left(\alpha_{t}\right)$ in notations of [14], [15]. For these markings, $G$ is denoted by $H$. They give a description of possible $\left(S, G, G\left(\alpha_{1}\right), \ldots G\left(\alpha_{t}\right)\right)$ by integer lattices. For these markings, we denote by $*$ markings which imply uniqueness of the lattice $S$, up to isomorphisms, where we use ideas by Hashimoto [5] and our methods in [15] - 18]. Differently from [15] - 18], we give only part of analogous to [15] 18 markings by Niemeier lattices, which is sufficient for the description of lattices $S$ of degenerations $D e g$ (otherwise, there will be too many markings and cases). Moreover (analogous to methods which we discussed in [15] [18]), we also use degenerations of smaller codimensions and their markings by Niemeier lattices if the considered degeneration can be reduced to them because of calculations of geneses of lattices $S$ of the Table 1 . This significantly reduces the number of considered cases.

Additionally to Tables 1 and 2, in Table 3 we give the List 1 which is important for classification of Picard lattices $S$ of K3 surfaces (see [18]). In Table 1, we denote by $o$ (old) cases when the degeneration of K3 surfaces with symplectic automorphism group $\left(C_{2}\right)^{2}$ has, actually, the maximal finite symplectic automorphism group $G$ which contains $\left(C_{2}\right)^{2}$ and $|G|>\left|\left(C_{2}\right)^{2}\right|=4$. The group $G$ has less orbits and less codimension of the degeneration than $\left(C_{2}\right)^{2}$. For classification of Picard lattices $S$ of K3 surfaces with maximal finite symplectic automorphism group $\left(C_{2}\right)^{2}$, lines of Table 1 which are denoted by $o$ must be removed. In Table 3, we give the List 1 of all these cases (which is similar to the List 1 of [18]) where the degeneration of the group $\left(C_{2}\right)^{2}$ is shown to the left, and the group $G$ (defined by $\mathbf{n}$ as an abstract group) and its degeneration (classified in [16] - [19]) is shown to the right from the sign $\Longleftarrow$. Cases of Table 1 which are marked by $o$ are also very interesting, they are similar to the case of Kummer surfaces which give the degeneration of type $16 \mathbb{A}_{1}$ of K3 surfaces with zero Picard lattice and trivial symplectic automorphism group, but the symplectic automorphism group of a general Kummer surface is $\left(C_{2}\right)^{4}$. See [9], [18]. 
Table 1: Some types and lattices $S$ of degenerations of Kählerian K3 surfaces with the symplectic automorphism group $G=\left(C_{2}\right)^{2}$.

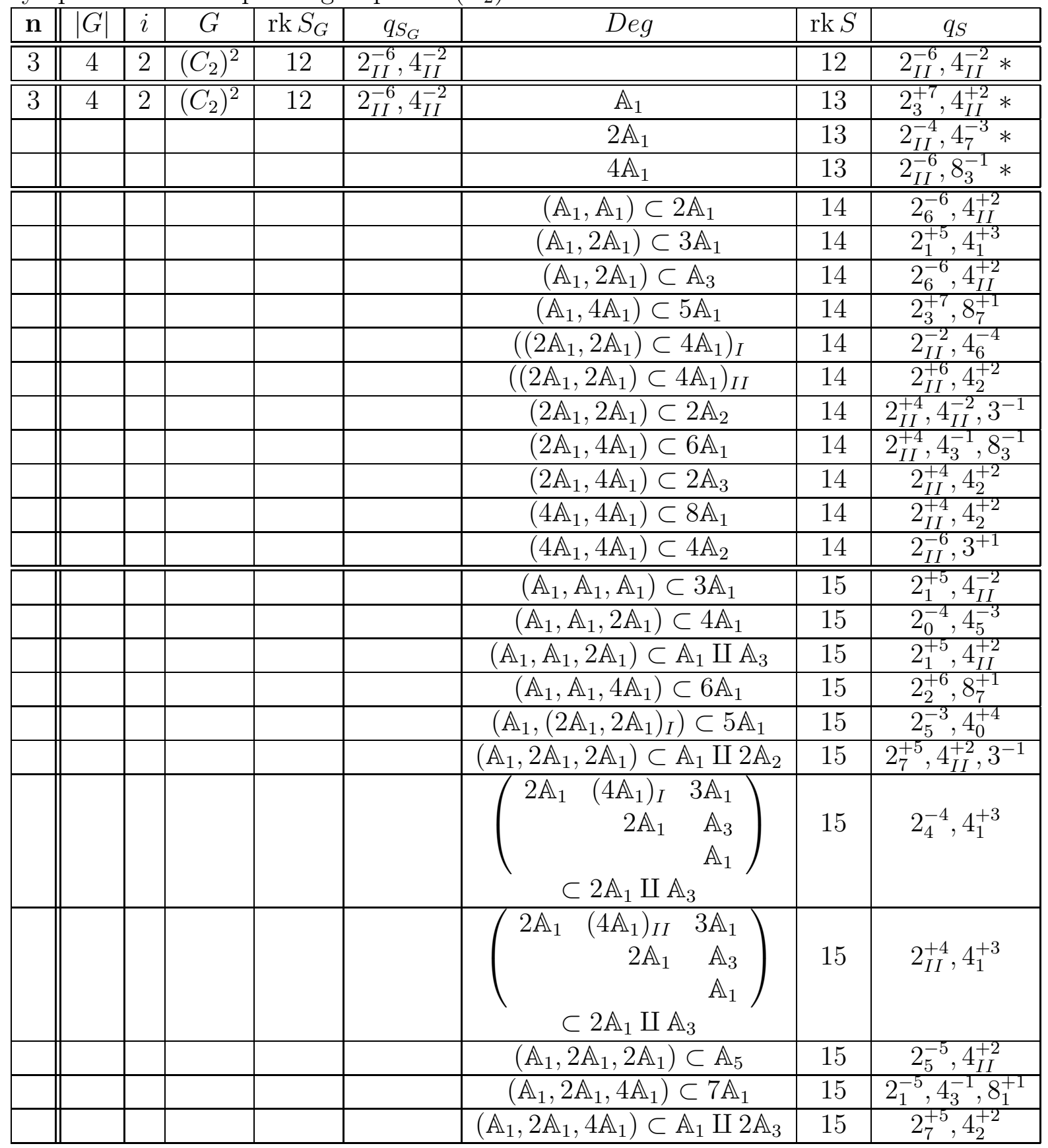




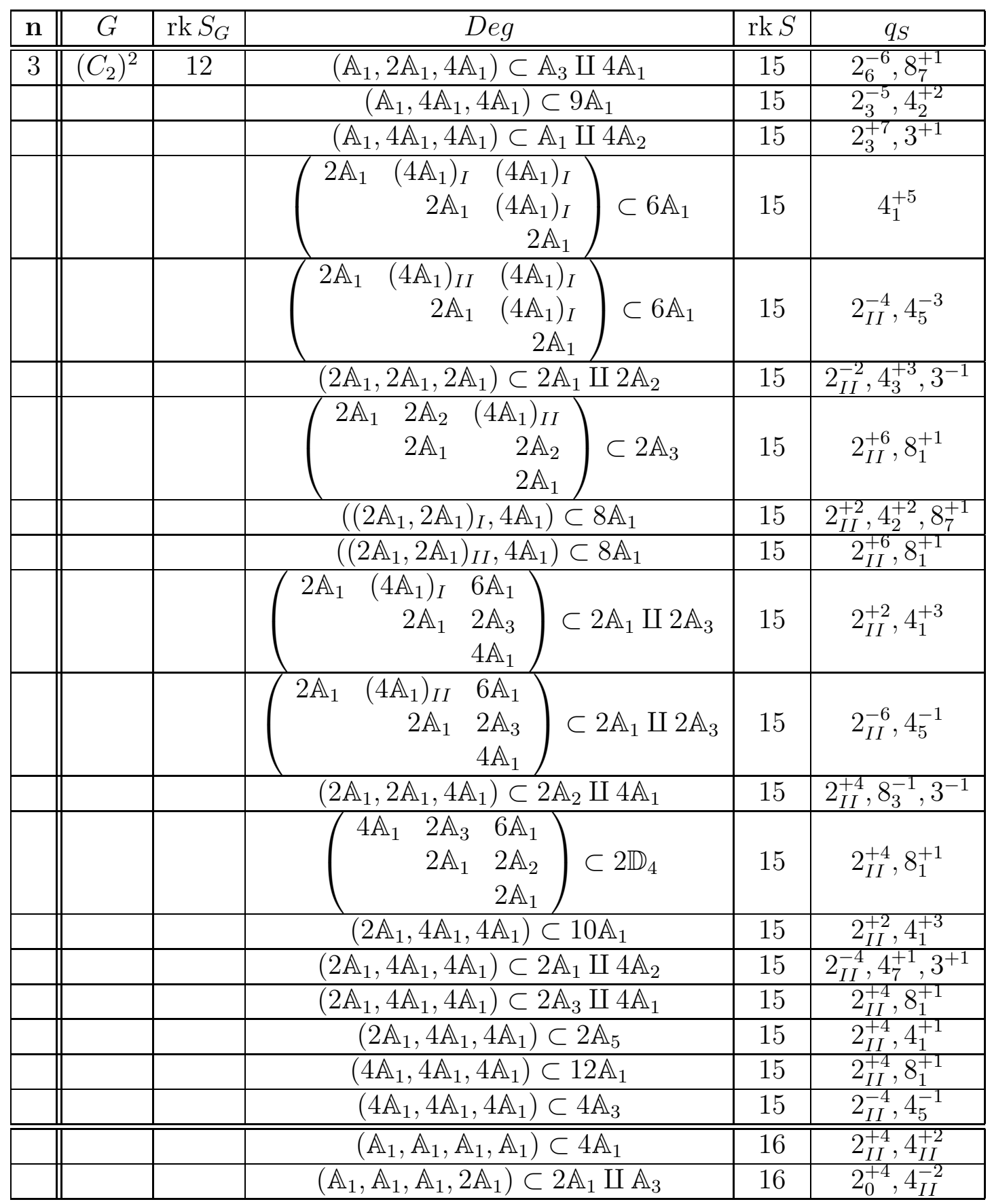




\begin{tabular}{|c|c|c|c|c|c|}
\hline $\mathbf{n}$ & $G$ & $\operatorname{rk} S_{G}$ & Deg & $\operatorname{rk} S$ & $q_{S}$ \\
\hline \multirow[t]{15}{*}{3} & $\overline{\left(C_{2}\right)^{2}}$ & 12 & $\left(\mathbb{A}_{1}, \mathbb{A}_{1}, \mathbb{A}_{1}, 4 \mathbb{A}_{1}\right) \subset 7 \mathbb{A}_{1}$ & 16 & $2_{1}^{+5}, 8_{7}^{+1}$ \\
\hline & & & $\left(\mathbb{A}_{1}, \mathbb{A}_{1},\left(2 \mathbb{A}_{1}, 2 \mathbb{A}_{1}\right)_{I}\right) \subset 6 \mathbb{A}_{1}$ & 16 & $2_{4}^{-2}, 4_{0}^{-4}$ \\
\hline & & & $\left(\begin{array}{rrrr}\mathbb{A}_{1} & 2 \mathbb{A}_{1} & 3 \mathbb{A}_{1} & 3 \mathbb{A}_{1} \\
& \mathbb{A}_{1} & \mathbb{A}_{3} & 3 \mathbb{A}_{1} \\
& & 2 \mathbb{A}_{1} & \left(4 \mathbb{A}_{1}\right)_{I} \\
& & & 2 \mathbb{A}_{1}\end{array}\right) \subset \mathbb{A}_{1} \amalg \mathbb{A}_{3} \amalg 2 \mathbb{A}_{1}$ & 16 & $2_{3}^{-3}, 4_{1}^{+3}$ \\
\hline & & & $\left(\begin{array}{rrrr}\mathbb{A}_{1} & \mathbb{A}_{3} & 2 \mathbb{A}_{1} & 3 \mathbb{A}_{1} \\
& 2 \mathbb{A}_{1} & 3 \mathbb{A}_{1} & \left(4 \mathbb{A}_{1}\right)_{I} \\
& & \mathbb{A}_{1} & \mathbb{A}_{3} \\
& & & 2 \mathbb{A}_{1}\end{array}\right)$ & 16 & $2_{0}^{+4}, 4_{I I}^{+2}$ \\
\hline & & & $\left.\begin{array}{ccrr}\mathbb{A}_{1} & \mathbb{A}_{3} & 2 \mathbb{A}_{1} & 3 \mathbb{A}_{1} \\
& 2 \mathbb{A}_{1} & 3 \mathbb{A}_{1} & \left(4 \mathbb{A}_{1}\right)_{I I} \\
& & \mathbb{A}_{1} & \mathbb{A}_{3} \\
& & & 2 \mathbb{A}_{1}\end{array}\right)$ & 16 & $2_{I I}^{+4}, 4_{I I}^{+2}$ \\
\hline & & & $\left(2 \mathbb{A}_{1}, 2 \mathbb{A}_{1}, \mathbb{A}_{1}, \mathbb{A}_{1}\right) \subset \mathbb{A}_{5} \amalg \mathbb{A}_{1}$ & 16 & $2_{4}^{-4}, 4_{I I}^{-2}$ \\
\hline & & & $\left(\mathbb{A}_{1}, \mathbb{A}_{1}, 2 \mathbb{A}_{1}, 4 \mathbb{A}_{1}\right) \subset 8 \mathbb{A}_{1}$ & 16 & $2_{0}^{+4}, 4_{1}^{+1}, 8_{3}^{-1}$ \\
\hline & & & $\left(\mathbb{A}_{1}, \mathbb{A}_{1}, 2 \mathbb{A}_{1}, 4 \mathbb{A}_{1}\right) \subset 2 \mathbb{A}_{1} \amalg 2 \mathbb{A}_{3}$ & 16 & $2_{2}^{-4}, 4_{2}^{+2}$ \\
\hline & & & $\left(\mathbb{A}_{1}, \mathbb{A}_{1}, 2 \mathbb{A}_{1}, 4 \mathbb{A}_{1}\right) \subset \mathbb{A}_{1} \amalg \mathbb{A}_{3} \amalg 4 \mathbb{A}_{1}$ & 16 & $2_{1}^{+5}, 8_{7}^{+1}$ \\
\hline & & & $\left(\mathbb{A}_{1}, \mathbb{A}_{1}, 4 \mathbb{A}_{1}, 4 \mathbb{A}_{1}\right)_{I} \subset 10 \mathbb{A}_{1}$ & 16 & $2_{0}^{+4}, 4_{0}^{+2}$ \\
\hline & & & $\left(\mathbb{A}_{1}, \mathbb{A}_{1}, 4 \mathbb{A}_{1}, 4 \mathbb{A}_{1}\right)_{I I} \subset 10 \mathbb{A}_{1}$ & 16 & $2_{I I}^{+4}, 4_{0}^{+2}$ \\
\hline & & & $\left(\mathbb{A}_{1}, \mathbb{A}_{1}, 4 \mathbb{A}_{1}, 4 \mathbb{A}_{1}\right) \subset 2 \mathbb{A}_{1} \amalg 4 \mathbb{A}_{2}$ & 16 & $2_{6}^{-6}, 3^{+1}$ \\
\hline & & & $\left(\begin{array}{rrrr}\mathbb{A}_{1} & 3 \mathbb{A}_{1} & 3 \mathbb{A}_{1} & 3 \mathbb{A}_{1} \\
& 2 \mathbb{A}_{1} & \left(4 \mathbb{A}_{1}\right)_{I} & \left(4 \mathbb{A}_{1}\right)_{I} \\
& & 2 \mathbb{A}_{1} & \left(4 \mathbb{A}_{1}\right)_{I} \\
& & & 2 \mathbb{A}_{1}\end{array}\right) \subset 7 \mathbb{A}_{1}$ & 16 & $2_{1}^{+1}, 4_{7}^{+5}$ \\
\hline & & & $\left(\mathbb{A}_{1}, 2 \mathbb{A}_{1}, 2 \mathbb{A}_{1}, 2 \mathbb{A}_{1}\right) \subset 3 \mathbb{A}_{1} \amalg 2 \mathbb{A}_{2}$ & 16 & $2_{5}^{+3}, 4_{1}^{+3}, 3^{-1}$ \\
\hline & & & $\left(\begin{array}{rrrr}\mathbb{A}_{1} & \mathbb{A}_{3} & 3 \mathbb{A}_{1} & 3 \mathbb{A}_{1} \\
& 2 \mathbb{A}_{1} & \left(4 \mathbb{A}_{1}\right)_{I} & \left(4 \mathbb{A}_{1}\right)_{I} \\
& & 2 \mathbb{A}_{1} & \left(4 \mathbb{A}_{1}\right)_{I} \\
& & & 2 \mathbb{A}_{1}\end{array}\right) \subset \mathbb{A}_{3} \amalg 4 \mathbb{A}_{1}$ & 16 & $2_{4}^{-2}, 4_{0}^{+4}$ \\
\hline
\end{tabular}




\begin{tabular}{|c|c|c|c|c|c|c|}
\hline n & $G$ & $\operatorname{rks} S_{G}$ & $\overline{D e g}$ & & rk $S$ & $q_{S}$ \\
\hline \multirow[t]{9}{*}{3} & $\left(C_{2}\right)^{2}$ & 12 & $\begin{array}{rrrr}\mathbb{A}_{1} & \mathbb{A}_{3} & 3 \mathbb{A}_{1} & 3 \mathbb{A}_{1} \\
& 2 \mathbb{A}_{1} & \left(4 \mathbb{A}_{1}\right)_{I I} & \left(4 \mathbb{A}_{1}\right)_{I} \\
& & 2 \mathbb{A}_{1} & \left(4 \mathbb{A}_{1}\right)_{I} \\
& & & 2 \mathbb{A}_{1}\end{array}$ & $\subset \mathbb{A}_{3} \amalg 4 \mathbb{A}_{1}$ & 16 & $2_{I I}^{+2}, 4_{0}^{+4}$ \\
\hline & & & \begin{tabular}{|rrrr}
$\mathbb{A}_{1}$ & $\mathbb{A}_{3}$ & $3 \mathbb{A}_{1}$ & $3 \mathbb{A}_{1}$ \\
& $2 \mathbb{A}_{1}$ & $\left(4 \mathbb{A}_{1}\right)_{I}$ & $\left(4 \mathbb{A}_{1}\right)_{I}$ \\
& & $2 \mathbb{A}_{1}$ & $2 \mathbb{A}_{2}$ \\
& & & $2 \mathbb{A}_{1}$ \\
\end{tabular} & $\subset \mathbb{A}_{3} \amalg 2 \mathbb{A}_{2}$ & 16 & $2_{6}^{+4}, 4_{I I}^{+2}, 3^{-1}$ \\
\hline & & & 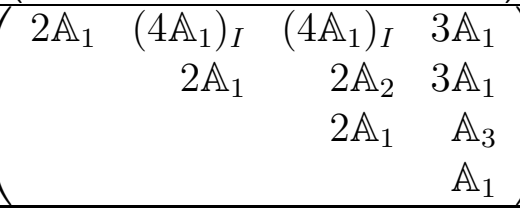 & $\subset 2 \mathbb{A}_{1} \amalg \mathbb{A}_{5}$ & 16 & $2_{5}^{+3}, 4_{3}^{-3}$ \\
\hline & & & $\left(\begin{array}{rrrr}2 \mathbb{A}_{1} & 2 \mathbb{A}_{2} & \left(4 \mathbb{A}_{1}\right)_{I I} & 3 \mathbb{A} \\
& 2 \mathbb{A}_{1} & 2 \mathbb{A}_{2} & 3 \mathbb{A} \\
& & 2 \mathbb{A}_{1} & \mathbb{A} \\
& & & \mathbb{A} \\
& & & \end{array}\right.$ & $\left.\begin{array}{l}1 \\
1 \\
3 \\
1\end{array}\right) \subset \mathbb{A}_{7}$ & 16 & $2_{I I}^{+4}, 4_{I I}^{+2}$ \\
\hline & & & $\left(\mathbb{A}_{1},\left(2 \mathbb{A}_{1}, 2 \mathbb{A}_{1}\right)_{I}, 4 \mathbb{A}_{1}\right)$ & $\subset 9 \mathbb{A}_{1}$ & 16 & $2_{7}^{+3}, 4_{2}^{+2}, 8_{3}^{-1}$ \\
\hline & & & $\left(\mathbb{A}_{1}, 2 \mathbb{A}_{1}, 2 \mathbb{A}_{1}, 4 \mathbb{A}_{1}\right) \subset \mathbb{A}_{1} \amalg$ & $2 \mathbb{A}_{2} \amalg 4 \mathbb{A}_{1}$ & 16 & $2_{3}^{-5}, 8_{3}^{-1}, 3^{-1}$ \\
\hline & & & $\left(\begin{array}{rrrr}\mathbb{A}_{1} & 3 \mathbb{A}_{1} & 3 \mathbb{A}_{1} & 5 \mathbb{A}_{1} \\
& 2 \mathbb{A}_{1} & \left(4 \mathbb{A}_{1}\right)_{I} & 6 \mathbb{A}_{1} \\
& & 2 \mathbb{A}_{1} & 2 \mathbb{A}_{3} \\
& & & 4 \mathbb{A}_{1}\end{array}\right)$ & $\subset 3 \mathbb{A}_{1} \amalg 2 \mathbb{A}_{3}$ & 16 & $2_{3}^{-3}, 4_{1}^{+3}$ \\
\hline & & & $\left(\begin{array}{rrrr}\mathbb{A}_{1} & \mathbb{A}_{3} & 3 \mathbb{A}_{1} & 5 \mathbb{A}_{1} \\
& 2 \mathbb{A}_{1} & \left(4 \mathbb{A}_{1}\right)_{I} & 6 \mathbb{A}_{1} \\
& & 2 \mathbb{A}_{1} & 6 \mathbb{A}_{1} \\
& & & 4 \mathbb{A}_{1}\end{array}\right)$ & $\subset \mathbb{A}_{3} \amalg 6 \mathbb{A}_{1}$ & 16 & $2_{6}^{-4}, 4_{7}^{+1}, 8_{7}^{+1}$ \\
\hline & & & $\left(\begin{array}{rrrr}\mathbb{A}_{1} & \mathbb{A}_{3} & 3 \mathbb{A}_{1} & 5 \mathbb{A}_{1} \\
& 2 \mathbb{A}_{1} & \left(4 \mathbb{A}_{1}\right)_{I I} & 6 \mathbb{A}_{1} \\
& & 2 \mathbb{A}_{1} & 6 \mathbb{A}_{1} \\
& & & 4 \mathbb{A}_{1}\end{array}\right)$ & $\subset \mathbb{A}_{3} \amalg 6 \mathbb{A}_{1}$ & 16 & $2_{I I}^{+4}, 4_{1}^{+1}, 8_{7}^{+1}$ \\
\hline
\end{tabular}




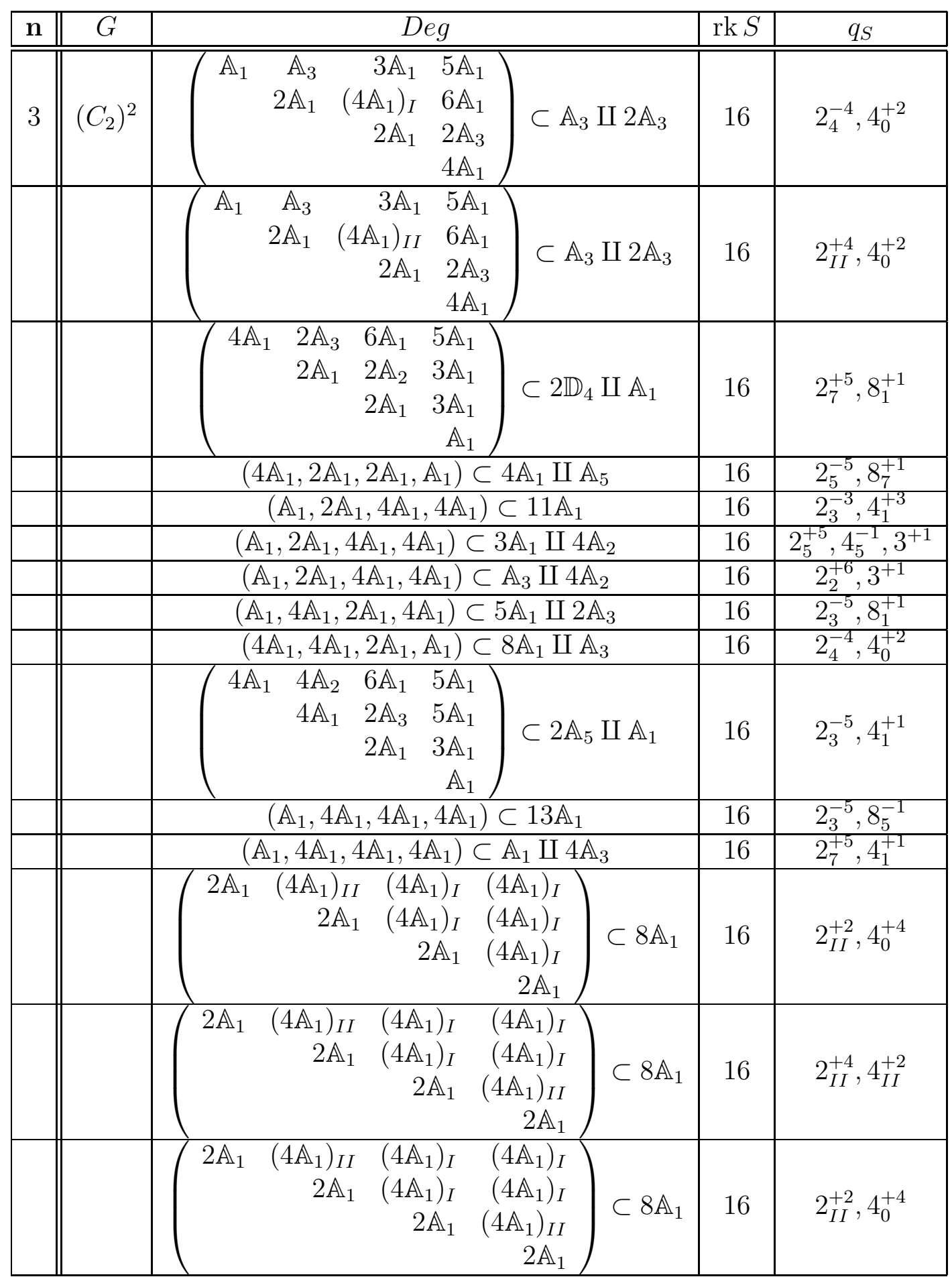




\begin{tabular}{|c|c|c|c|c|c|}
\hline $\mathbf{n}$ & $G$ & \multicolumn{2}{|l|}{$D e g$} & $\operatorname{rk} S$ & $q_{S}$ \\
\hline 3 & $\left(C_{2}\right)^{2}$ & $\begin{array}{rrrr}2 \mathbb{A}_{1} & \left(4 \mathbb{A}_{1}\right)_{I} & \left(4 \mathbb{A}_{1}\right)_{I} & \left(4 \mathbb{A}_{1}\right)_{I} \\
& 2 \mathbb{A}_{1} & 2 \mathbb{A}_{2} & \left(4 \mathbb{A}_{1}\right)_{I} \\
& & 2 \mathbb{A}_{1} & \left(4 \mathbb{A}_{1}\right)_{I} \\
& & & 2 \mathbb{A}_{1}\end{array}$ & $\subset 2 \mathbb{A}_{1} \amalg 2 \mathbb{A}_{2} \amalg 2 \mathbb{A}_{1}$ & 16 & $4_{6}^{-4}, 3^{-1}$ \\
\hline & & $\left.\begin{array}{rrrr}2 \mathbb{A}_{1} & \left(4 \mathbb{A}_{1}\right)_{I} & \left(4 \mathbb{A}_{1}\right)_{I} & \left(4 \mathbb{A}_{1}\right)_{I I} \\
& 2 \mathbb{A}_{1} & 2 \mathbb{A}_{2} & \left(4 \mathbb{A}_{1}\right)_{I} \\
& & 2 \mathbb{A}_{1} & \left(4 \mathbb{A}_{1}\right)_{I} \\
& & & 2 \mathbb{A}_{1}\end{array}\right)$ & $\subset 2 \mathbb{A}_{1} \amalg 2 \mathbb{A}_{2} \amalg 2 \mathbb{A}_{1}$ & 16 & $2_{I I}^{-4}, 4_{2}^{+2}, 3^{-1}$ \\
\hline & & \multicolumn{2}{|c|}{$\left(2 \mathbb{A}_{1}, 2 \mathbb{A}_{1}, 2 \mathbb{A}_{1}, 2 \mathbb{A}_{1}\right) \subset 2 \mathbb{A}_{2} \amalg 2 \mathbb{A}_{2}$} & 16 & $2_{I I}^{-2}, 4_{I I}^{-2}, 3^{+2}$ \\
\hline & & $\left(\begin{array}{rrrr}2 \mathbb{A}_{1} & \left(4 \mathbb{A}_{1}\right)_{I} & \left(4 \mathbb{A}_{1}\right)_{I} & \left(4 \mathbb{A}_{1}\right)_{I} \\
& 2 \mathbb{A}_{1} & 2 \mathbb{A}_{2} & \left(4 \mathbb{A}_{1}\right)_{I I} \\
& 2 \mathbb{A}_{1} & 2 \mathbb{A}_{2} \\
& & & 2 \mathbb{A}_{1}\end{array}\right.$ & $\subset 2 \mathbb{A}_{1} \amalg 2 \mathbb{A}_{3}$ & 16 & $2_{I I}^{+4}, 4_{7}^{+1}, 8_{1}^{+1}$ \\
\hline & & $\left(\begin{array}{rrrr}2 \mathbb{A}_{1} & \left(4 \mathbb{A}_{1}\right)_{I} & \left(4 \mathbb{A}_{1}\right)_{I} & 6 \mathbb{A} \\
& 2 \mathbb{A}_{1} & \left(4 \mathbb{A}_{1}\right)_{I} & 6 \mathbb{A} \\
& & 2 \mathbb{A}_{1} & 6 \mathbb{A} \\
& & & 4 \mathbb{A} \\
& & & \\
& & \end{array}\right.$ & $\subset 10 \mathbb{A}_{1}$ & 16 & $4_{7}^{-3}, 8_{5}^{-1}$ \\
\hline & & $\left(\begin{array}{rrrr}2 \mathbb{A}_{1} & \left(4 \mathbb{A}_{1}\right)_{I I} & \left(4 \mathbb{A}_{1}\right)_{I} & 6 \AA \\
& 2 \mathbb{A}_{1} & \left(4 \mathbb{A}_{1}\right)_{I} & 6 \AA \\
& & 2 \mathbb{A}_{1} & 6 A \\
& & & 4 \AA\end{array}\right.$ & $\left.\begin{array}{l}\mathbb{A}_{1} \\
\mathbb{A}_{1} \\
\mathbb{A}_{1}\end{array}\right)$ & 16 & $2_{I I}^{-4}, 4_{3}^{-1}, 8_{1}^{+1}$ \\
\hline & & $\left(\begin{array}{ccrr}4 \mathbb{A}_{1} & 2 \mathbb{A}_{3} & 6 \mathbb{A}_{1} & 6 \mathbb{A}_{1} \\
& 2 \mathbb{A}_{1} & \left(4 \mathbb{A}_{1}\right)_{I} & \left(4 \mathbb{A}_{1}\right)_{I} \\
& & 2 \mathbb{A}_{1} & \left(4 \mathbb{A}_{1}\right)_{I} \\
& & & 2 \mathbb{A}_{1}\end{array}\right.$ & $\subset 2 \mathbb{A}_{3} \amalg 4 \mathbb{A}_{1}$ & 16 & $4_{0}^{+4}$ \\
\hline & & $\left(\begin{array}{ccrr}4 \mathbb{A}_{1} & 2 \mathbb{A}_{3} & 6 \mathbb{A}_{1} & 6 \mathbb{A}_{1} \\
& 2 \mathbb{A}_{1} & \left(4 \mathbb{A}_{1}\right)_{I I} & \left(4 \mathbb{A}_{1}\right)_{I} \\
& & 2 \mathbb{A}_{1} & \left(4 \mathbb{A}_{1}\right)_{I} \\
& & & 2 \mathbb{A}_{1}\end{array}\right.$ & $\subset 2 \mathbb{A}_{3} \amalg 4 \mathbb{A}_{1}$ & 16 & $2_{I I}^{-4}, 4_{4}^{-2}$ \\
\hline & & $\begin{array}{rrrr}4 \mathbb{A}_{1} & 2 \mathbb{A}_{3} & 6 \mathbb{A}_{1} & 6 \mathbb{A}_{1} \\
& 2 \mathbb{A}_{1} & \left(4 \mathbb{A}_{1}\right)_{I} & \left(4 \mathbb{A}_{1}\right)_{I} \\
& & 2 \mathbb{A}_{1} & \left(4 \mathbb{A}_{1}\right)_{I I} \\
& & & 2 \mathbb{A}_{1}\end{array}$ & $\subset 2 \mathbb{A}_{3} \amalg 4 \mathbb{A}_{1}$ & 16 & $2_{I I}^{+4}, 4_{I I}^{+2}$ \\
\hline
\end{tabular}




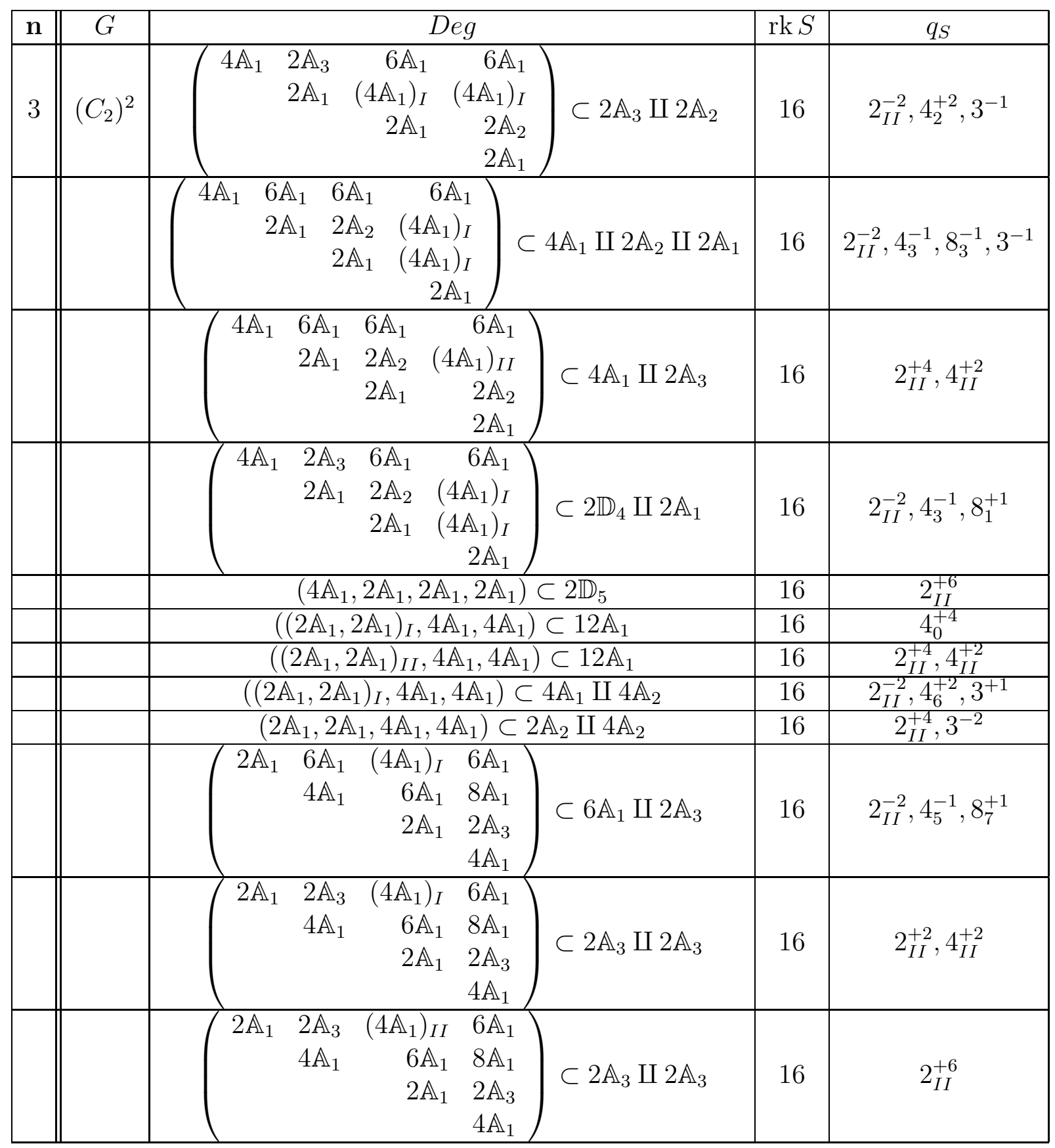




\begin{tabular}{|c|c|c|c|c|c|}
\hline $\mathbf{n}$ & $G$ & \multicolumn{2}{|l|}{ Deg } & $\operatorname{rk} S$ & $q_{S}$ \\
\hline 3 & $\left(C_{2}\right)^{2}$ & $\left(\begin{array}{cccc}4 \mathbb{A}_{1} & 2 \mathbb{A}_{3} & 6 \mathbb{A}_{1} & 8 \mathbb{A}_{1} \\
& 2 \mathbb{A}_{1} & 2 \mathbb{A}_{2} & 6 \mathbb{A}_{1} \\
& & 2 \mathbb{A}_{1} & 6 \mathbb{A}_{1} \\
& & & 4 \mathbb{A}_{1}\end{array}\right)$ & $\subset 2 \mathbb{D}_{4} \amalg 4 \mathbb{A}_{1}$ & 16 & $2_{I I}^{+2}, 4_{I I}^{+2}$ \\
\hline & & \begin{tabular}{|cccr}
$4 \mathbb{A}_{1}$ & $4 \mathbb{A}_{2}$ & $6 \mathbb{A}_{1}$ & $6 \mathbb{A}_{1}$ \\
& $4 \mathbb{A}_{1}$ & $2 \mathbb{A}_{3}$ & $6 \mathbb{A}_{1}$ \\
& & $2 \mathbb{A}_{1}$ & $\left(4 \mathbb{A}_{1}\right)_{I}$ \\
& & & $2 \mathbb{A}_{1}$ \\
\end{tabular} & $\subset 2 \mathbb{A}_{5} \amalg 2 \mathbb{A}_{1}$ & 16 & $2_{I I}^{+2}, 4_{0}^{+2}$ \\
\hline & & $\begin{array}{|cccr|}4 \mathbb{A}_{1} & 4 \mathbb{A}_{2} & 6 \mathbb{A}_{1} & 6 \mathbb{A}_{1} \\
& 4 \mathbb{A}_{1} & 2 \mathbb{A}_{3} & 6 \mathbb{A}_{1} \\
& & 2 \mathbb{A}_{1} & \left(4 \mathbb{A}_{1}\right)_{I I} \\
& & & 2 \mathbb{A}_{1} \\
\end{array}$ & $\subset 2 \mathbb{A}_{5} \amalg 2 \mathbb{A}_{1}$ & 16 & $2_{I I}^{+6}$ \\
\hline & & $\left(4 \mathbb{A}_{1}, 4 \mathbb{A}_{1}, 2 \mathbb{A}_{1}, 2 \mathbb{A}_{1}\right)$ & $\subset 2 \mathbb{E}_{6}$ & 16 & $2_{I I}^{+4}$ \\
\hline & & $\left(2 \mathbb{A}_{1}, 4 \mathbb{A}_{1}, 4 \mathbb{A}_{1}, 4 \mathbb{A}_{1}\right) \subset 2$ & $2 \mathbb{A}_{3} \amalg 8 \mathbb{A}_{1}$ & 16 & $2_{I I}^{+2}, 4_{I I}^{+2}$ \\
\hline & & $\left(2 \mathbb{A}_{1}, 4 \mathbb{A}_{1}, 4 \mathbb{A}_{1}, 4 \mathbb{A}_{1}\right) \subset 2$ & $2 \mathbb{A}_{1} \amalg 4 \mathbb{A}_{3}$ & 16 & $2_{I I}^{-2}, 4_{4}^{-2}$ \\
\hline & & $\left(\begin{array}{cccc}4 \mathbb{A}_{1} & 4 \mathbb{A}_{2} & 8 \mathbb{A}_{1} & 6 \mathbb{A}_{1} \\
& 4 \mathbb{A}_{1} & 4 \mathbb{A}_{2} & 6 \mathbb{A}_{1} \\
& & 4 \mathbb{A}_{1} & 2 \mathbb{A}_{3} \\
& & & 2 \mathbb{A}_{1}\end{array}\right.$ & $\subset 2 \mathbb{A}_{7}$ & 16 & $2_{I I}^{+4}$ \\
\hline & & $\left(\begin{array}{cccc}4 \mathbb{A}_{1} & 4 \mathbb{A}_{2} & 8 \mathbb{A}_{1} & 8 \mathbb{A}_{1} \\
& 4 \mathbb{A}_{1} & 4 \mathbb{A}_{2} & 4 \mathbb{A}_{2} \\
& & 4 \mathbb{A}_{1} & 8 \mathbb{A}_{1} \\
& & & 4 \mathbb{A}_{1} \\
& & & \end{array}\right.$ & $\subset 4 \mathbb{D}_{4}$ & 16 & $2_{I I}^{+4}$ \\
\hline & & $\left(4 \mathbb{A}_{1}, 4 \mathbb{A}_{1}, 4 \mathbb{A}_{1}, 4 \mathbb{A}_{1}\right)$ & $\subset 16 \mathbb{A}_{1}$ & 16 & $2_{I I}^{+6}$ \\
\hline
\end{tabular}




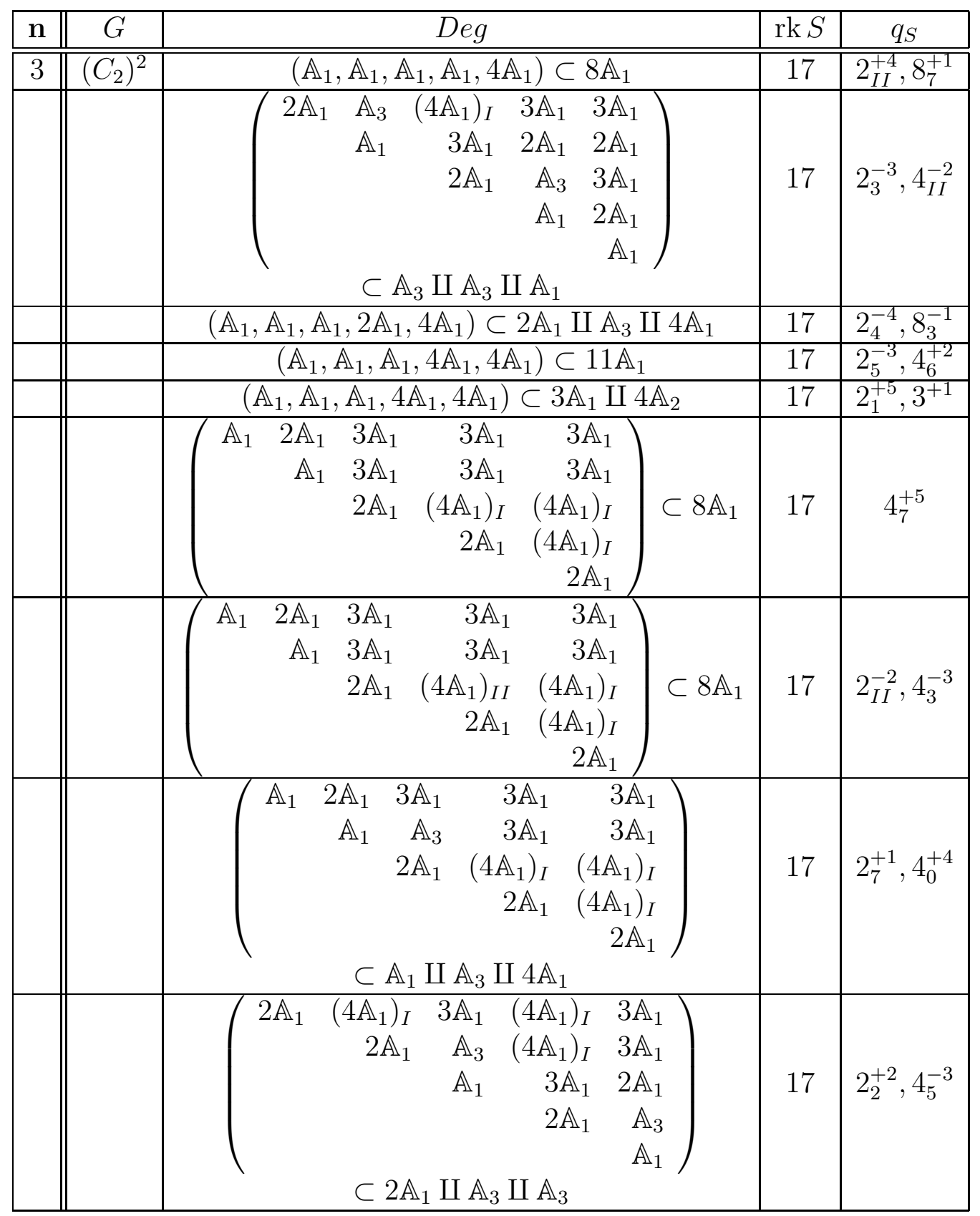




\begin{tabular}{|c|c|c|c|c|}
\hline n & $\bar{G}$ & $\overline{D e g}$ & rk $S$ & $q_{S}$ \\
\hline 3 & $\left(C_{2}\right)^{2}$ & $\left(\begin{array}{rrrrr}2 \mathbb{A}_{1} & \left(4 \mathbb{A}_{1}\right)_{I} & 3 \mathbb{A}_{1} & \left(4 \mathbb{A}_{1}\right)_{I} & 3 \mathbb{A}_{1} \\
& 2 \mathbb{A}_{1} & \mathbb{A}_{3} & \left(4 \mathbb{A}_{1}\right)_{I I} & 3 \mathbb{A}_{1} \\
& & \mathbb{A}_{1} & 3 \mathbb{A}_{1} & 2 \mathbb{A}_{1} \\
& & & 2 \mathbb{A}_{1} & \mathbb{A}_{3} \\
& & & & \mathbb{A}_{1}\end{array}\right)$ & 17 & $2_{I I}^{+2}, 4_{7}^{+3}$ \\
\hline & & $\left(\begin{array}{rrrrr}2 \mathbb{A}_{1} & \left(4 \mathbb{A}_{1}\right)_{I} & \left(4 \mathbb{A}_{1}\right)_{I} & 3 \mathbb{A}_{1} & 3 \mathbb{A}_{1} \\
& 2 \mathbb{A}_{1} & 2 \mathbb{A}_{2} & 3 \mathbb{A}_{1} & 3 \mathbb{A}_{1} \\
& & 2 \mathbb{A}_{1} & \mathbb{A}_{3} & 3 \mathbb{A}_{1} \\
& & & \mathbb{A}_{1} & 2 \mathbb{A}_{1} \\
& & & & \mathbb{A}_{1}\end{array}\right)$ & 17 & $2_{0}^{+2}, 4_{7}^{-3}$ \\
\hline & & $\left.\begin{array}{ccccc}2 \mathbb{A}_{1} & 2 \mathbb{A}_{2} & 3 \mathbb{A}_{1} & \left(4 \mathbb{A}_{1}\right)_{I} & 3 \mathbb{A}_{1} \\
& 2 \mathbb{A}_{1} & \mathbb{A}_{3} & \left(4 \mathbb{A}_{1}\right)_{I} & 3 \mathbb{A}_{1} \\
& & \mathbb{A}_{1} & 3 \mathbb{A}_{3} & 3 \mathbb{A}_{1} \\
& & & 2 \mathbb{A}_{1} & \mathbb{A}_{3} \\
& & & & \mathbb{A}_{1}\end{array}\right)$ & 17 & $2_{3}^{-3}, 4_{I I}^{+2}$ \\
\hline & & $\left(\mathbb{A}_{1}, \mathbb{A}_{1},\left(2 \mathbb{A}_{1}, 2 \mathbb{A}_{1}\right)_{I}, 4 \mathbb{A}_{1}\right) \subset 10 \mathbb{A}_{1}$ & 17 & $2_{6}^{-2}, 4_{6}^{-2}, 8_{3}^{-1}$ \\
\hline & & 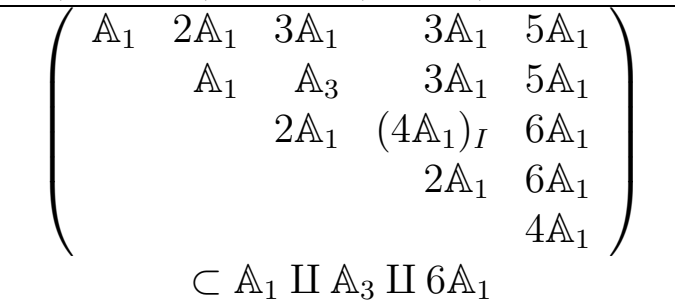 & 17 & $2_{1}^{-3}, 4_{3}^{-1}, 8_{7}^{+1}$ \\
\hline & & 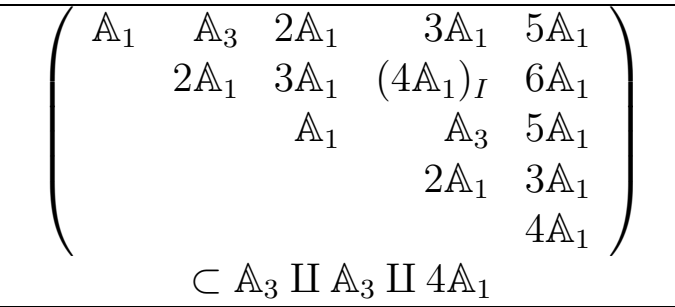 & 17 & $2_{0}^{+4}, 8_{7}^{+1}$ \\
\hline
\end{tabular}




\begin{tabular}{|c|c|c|c|c|}
\hline $\mathbf{n}$ & $G$ & Deg & rk $S$ & $q_{S}$ \\
\hline 3 & $\left(C_{2}\right)^{2}$ & $\left(\begin{array}{rrrrr}\mathbb{A}_{1} & \mathbb{A}_{3} & 2 \mathbb{A}_{1} & 3 \mathbb{A}_{1} & 5 \mathbb{A}_{1} \\
& 2 \mathbb{A}_{1} & 3 \mathbb{A}_{1} & \left(4 \mathbb{A}_{1}\right)_{I I} & 6 \mathbb{A}_{1} \\
& & \mathbb{A}_{1} & \mathbb{A}_{3} & 5 \mathbb{A}_{1} \\
& & & 2 \mathbb{A}_{1} & 3 \mathbb{A}_{1} \\
& & & & 4 \mathbb{A}_{1}\end{array}\right)$ & 17 & $2_{I I}^{+4}, 8_{7}^{+1}$ \\
\hline & & $\left(\begin{array}{rrrrr}\mathbb{A}_{1} & 2 \mathbb{A}_{1} & 3 \mathbb{A}_{1} & 3 \mathbb{A}_{1} & 5 \mathbb{A}_{1} \\
& \mathbb{A}_{1} & 3 \mathbb{A}_{1} & 3 \mathbb{A}_{1} & 5 \mathbb{A}_{1} \\
& & 2 \mathbb{A}_{1} & \left(4 \mathbb{A}_{1}\right)_{I} & 6 \mathbb{A}_{1} \\
& & & 2 \mathbb{A}_{1} & 2 \mathbb{A}_{3} \\
& & & & 4 \mathbb{A}_{1}\end{array}\right) \subset 4 \mathbb{A}_{1} \amalg 2 \mathbb{A}_{3}$ & 17 & $2_{6}^{+2}, 4_{1}^{+3}$ \\
\hline & & $\left(\begin{array}{rrrrr}\mathbb{A}_{1} & 2 \mathbb{A}_{1} & 3 \mathbb{A}_{1} & 3 \mathbb{A}_{1} & 5 \mathbb{A}_{1} \\
& \mathbb{A}_{1} & \mathbb{A}_{3} & 3 \mathbb{A}_{1} & 5 \mathbb{A}_{1} \\
& & 2 \mathbb{A}_{1} & \left(4 \mathbb{A}_{1}\right)_{I} & 6 \mathbb{A}_{1} \\
& & & 2 \mathbb{A}_{1} & 2 \mathbb{A}_{3} \\
& & & & 4 \mathbb{A}_{1}\end{array}\right)$ & 17 & $2_{7}^{+3}, 4_{0}^{+2}$ \\
\hline & & $\left(\mathbb{A}_{1}, 2 \mathbb{A}_{1}, 2 \mathbb{A}_{1}, \mathbb{A}_{1}, 4 \mathbb{A}_{1}\right) \subset \mathbb{A}_{1} \amalg \mathbb{A}_{5} \amalg 4 \mathbb{A}_{1}$ & 17 & $2_{0}^{+4}, 8_{7}^{+1}$ \\
\hline & & $\left(\mathbb{A}_{1}, \mathbb{A}_{1}, 2 \mathbb{A}_{1}, 4 \mathbb{A}_{1}, 4 \mathbb{A}_{1}\right) \subset 12 \mathbb{A}_{1}$ & 17 & $2_{6}^{+2}, 4_{1}^{+3}$ \\
\hline & & $\left(\mathbb{A}_{1}, \mathbb{A}_{1}, 2 \mathbb{A}_{1}, 4 \mathbb{A}_{1}, 4 \mathbb{A}_{1}\right) \subset 12 \mathbb{A}_{1}$ & 17 & $2_{I I}^{+2}, 4_{7}^{+3}$ \\
\hline & & $\left(\mathbb{A}_{1}, \mathbb{A}_{1}, 2 \mathbb{A}_{1}, 4 \mathbb{A}_{1}, 4 \mathbb{A}_{1}\right) \subset 4 \mathbb{A}_{1} \amalg 4 \mathbb{A}_{2}$ & 17 & $2_{4}^{+4}, 4_{5}^{-1}, 3^{+1}$ \\
\hline & & $\left(\mathbb{A}_{1}, \mathbb{A}_{1}, 2 \mathbb{A}_{1}, 4 \mathbb{A}_{1}, 4 \mathbb{A}_{1}\right) \subset \mathbb{A}_{1} \amalg \mathbb{A}_{3} \amalg 8 \mathbb{A}_{1}$ & 17 & $2_{7}^{+3}, 4_{0}^{+2}$ \\
\hline & & $\left(\mathbb{A}_{1}, \mathbb{A}_{1}, 2 \mathbb{A}_{1}, 4 \mathbb{A}_{1}, 4 \mathbb{A}_{1}\right) \subset \mathbb{A}_{1} \amalg \mathbb{A}_{3} \amalg 4 \mathbb{A}_{2}$ & 17 & $2_{6}^{+4}, 4_{3}^{-1}, 3^{+1}$ \\
\hline & & $\left(\mathbb{A}_{1}, \mathbb{A}_{1}, 2 \mathbb{A}_{1}, 4 \mathbb{A}_{1}, 4 \mathbb{A}_{1}\right) \subset \mathbb{A}_{1} \amalg \mathbb{A}_{3} \amalg 4 \mathbb{A}_{2}$ & 17 & $2_{1}^{+5}, 3^{+1}$ \\
\hline & & $\left(\mathbb{A}_{1}, \mathbb{A}_{1}, 4 \mathbb{A}_{1}, 2 \mathbb{A}_{1}, 4 \mathbb{A}_{1}\right) \subset 6 \mathbb{A}_{1} \amalg 2 \mathbb{A}_{3}$ & 17 & $2_{6}^{+4}, 8_{1}^{+1}$ \\
\hline & & $\left(4 \mathbb{A}_{1}, 4 \mathbb{A}_{1}, 2 \mathbb{A}_{1}, \mathbb{A}_{1}, \mathbb{A}_{1}\right) \subset 2 \mathbb{A}_{5} \amalg 2 \mathbb{A}_{1}$ & 17 & $2_{2}^{+4}, 4_{5}^{-1}$ \\
\hline & & $\left(\mathbb{A}_{1}, \mathbb{A}_{1}, 4 \mathbb{A}_{1}, 4 \mathbb{A}_{1}, 4 \mathbb{A}_{1}\right) \subset 14 \mathbb{A}_{1}$ & 17 & $2_{2}^{-4}, 8_{5}^{-1}$ \\
\hline & & $\left(\mathbb{A}_{1}, \mathbb{A}_{1}, 4 \mathbb{A}_{1}, 4 \mathbb{A}_{1}, 4 \mathbb{A}_{1}\right) \subset 2 \mathbb{A}_{1} \amalg 4 \mathbb{A}_{3}$ & 17 & $2_{I I}^{+4}, 4_{7}^{+1}$ \\
\hline & & $\left(\mathbb{A}_{1},\left(2 \mathbb{A}_{1}, 2 \mathbb{A}_{1}\right)_{I}, 2 \mathbb{A}_{1}, 2 \mathbb{A}_{1}\right) \subset 5 \mathbb{A}_{1} \amalg 2 \mathbb{A}_{2}$ & 17 & $2_{5}^{-1}, 4_{4}^{-4}, 3^{-1}$ \\
\hline
\end{tabular}




\begin{tabular}{|c|c|c|c|c|}
\hline $\mathbf{n}$ & $G$ & Deg & $\operatorname{rk} S$ & $q_{S}$ \\
\hline 3 & $\left(C_{2}\right)^{2}$ & $\left.\begin{array}{ccrrr}\mathbb{A}_{1} & 2 \mathbb{A}_{2} & \left(4 \mathbb{A}_{1}\right)_{I} & \left(4 \mathbb{A}_{1}\right)_{I} & 3 \mathbb{A}_{1} \\
& 2 \mathbb{A}_{1} & \left(4 \mathbb{A}_{1}\right)_{I} & \left(4 \mathbb{A}_{1}\right)_{I} & 3 \mathbb{A}_{1} \\
& & 2 \mathbb{A}_{1} & \left(4 \mathbb{A}_{1}\right)_{I} & 3 \mathbb{A}_{1} \\
& & & 2 \mathbb{A}_{1} & \mathbb{A}_{3} \\
& & & & \mathbb{A}_{1}\end{array}\right)$ & 17 & $2_{6}^{-2}, 4_{3}^{-3}, 3^{-1}$ \\
\hline & & $\left(\begin{array}{rrrrr}2 \mathbb{A}_{1} & \left(4 \mathbb{A}_{1}\right)_{I} & \left(4 \mathbb{A}_{1}\right)_{I} & \left(4 \mathbb{A}_{1}\right)_{I I} & 3 \mathbb{A}_{1} \\
& 2 \mathbb{A}_{1} & 2 \mathbb{A}_{2} & \left(4 \mathbb{A}_{1}\right)_{I} & 3 \mathbb{A}_{1} \\
& & 2 \mathbb{A}_{1} & \left(4 \mathbb{A}_{1}\right)_{I} & 3 \mathbb{A}_{1} \\
& & & 2 \mathbb{A}_{1} & \mathbb{A}_{3} \\
& & & & \mathbb{A}_{1}\end{array}\right)$ & 17 & $2_{I I}^{+2}, 4_{5}^{-3}, 3^{-1}$ \\
\hline & & $\left(\mathbb{A}_{1}, 2 \mathbb{A}_{1}, 2 \mathbb{A}_{1}, 2 \mathbb{A}_{1}, 2 \mathbb{A}_{1}\right) \subset \mathbb{A}_{1} \amalg 2 \mathbb{A}_{2} \amalg 2 \mathbb{A}_{2}$ & 17 & $2_{3}^{+3}, 4_{I I}^{+2}, 3^{+2}$ \\
\hline & & $\left(\begin{array}{rrrrr}\mathbb{A}_{1} & \mathbb{A}_{3} & 3 \mathbb{A}_{1} & 3 \mathbb{A}_{1} & 3 \mathbb{A}_{1} \\
& 2 \mathbb{A}_{1} & \left(4 \mathbb{A}_{1}\right)_{I I} & \left(4 \mathbb{A}_{1}\right)_{I} & \left(4 \mathbb{A}_{1}\right)_{I} \\
& & 2 \mathbb{A}_{1} & \left(4 \mathbb{A}_{1}\right)_{I} & \left(4 \mathbb{A}_{1}\right)_{I} \\
& & & 2 \mathbb{A}_{1} & \left(4 \mathbb{A}_{1}\right)_{I} \\
& & & & 2 \mathbb{A}_{1}\end{array}\right)$ & 17 & $4_{7}^{+5}$ \\
\hline & & $\begin{array}{c}\left(\begin{array}{crrrr}2 \mathbb{A}_{1} & 2 \mathbb{A}_{2} & 3 \mathbb{A}_{1} & \left(4 \mathbb{A}_{1}\right)_{I} & \left(4 \mathbb{A}_{1}\right)_{I} \\
& 2 \mathbb{A}_{1} & \mathbb{A}_{3} & \left(4 \mathbb{A}_{1}\right)_{I} & \left(4 \mathbb{A}_{1}\right)_{I} \\
& & \mathbb{A}_{1} & 3 \mathbb{A}_{1} & 3 \mathbb{A}_{1} \\
& & & 2 \mathbb{A}_{1} & \left(4 \mathbb{A}_{1}\right)_{I} \\
& & & & \\
& & & & \\
& & & \\
& & \mathbb{A}_{5}\end{array}\right) \\
\end{array}$ & 17 & $2_{5}^{-1}, 4_{6}^{+4}$ \\
\hline & & $\left(\begin{array}{rrrrr}2 \mathbb{A}_{1} & 2 \mathbb{A}_{2} & 3 \mathbb{A}_{1} & \left(4 \mathbb{A}_{1}\right)_{I} & \left(4 \mathbb{A}_{1}\right)_{I} \\
& 2 \mathbb{A}_{1} & \mathbb{A}_{3} & \left(4 \mathbb{A}_{1}\right)_{I} & \left(4 \mathbb{A}_{1}\right)_{I} \\
& & \mathbb{A}_{1} & 3 \mathbb{A}_{1} & 3 \mathbb{A}_{1} \\
& & & 2 \mathbb{A}_{1} & 2 \mathbb{A}_{2} \\
& & & & 2 \mathbb{A}_{1}\end{array}\right)$ & 17 & $2_{1}^{-3}, 4_{I I}^{+2}, 3^{-1}$ \\
\hline
\end{tabular}




\begin{tabular}{|c|c|c|c|c|}
\hline $\mathrm{n}$ & $G$ & Deg & rk $S$ & $q_{S}$ \\
\hline \multirow[t]{6}{*}{3} & $\left(C_{2}\right)^{2}$ & 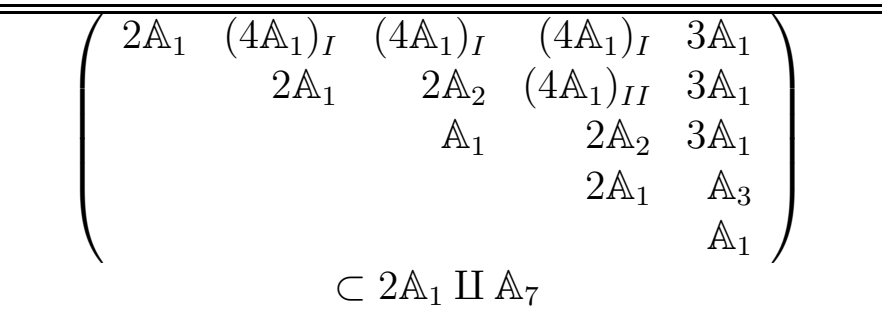 & 17 & $2_{I I}^{-2}, 4_{3}^{-3}$ \\
\hline & & $\left(\begin{array}{rrrrr}\mathbb{A}_{1} & 3 \mathbb{A}_{1} & 3 \mathbb{A}_{1} & 3 \mathbb{A}_{1} & 5 \mathbb{A}_{1} \\
& 2 \mathbb{A}_{1} & \left(4 \mathbb{A}_{1}\right)_{I} & \left(4 \mathbb{A}_{1}\right)_{I} & 6 \mathbb{A}_{1} \\
& & 2 \mathbb{A}_{1} & \left(4 \mathbb{A}_{1}\right)_{I} & 6 \mathbb{A}_{1} \\
& & & 2 \mathbb{A}_{1} & 6 \mathbb{A}_{1} \\
& & & & 4 \mathbb{A}_{1}\end{array}\right) \subset 11 \mathbb{A}_{1}$ & 17 & $2_{1}^{+1}, 4_{5}^{-3}, 8_{1}^{+1}$ \\
\hline & & $\left(\mathbb{A}_{1}, 2 \mathbb{A}_{1}, 2 \mathbb{A}_{1}, 2 \mathbb{A}_{1}, 4 \mathbb{A}_{1}\right) \subset 3 \mathbb{A}_{1} \amalg 2 \mathbb{A}_{2} \amalg 4 \mathbb{A}_{1}$ & 17 & $2_{3}^{-3}, 4_{5}^{-1}, 8_{5}^{-1}, 3^{-1}$ \\
\hline & & $\left.\begin{array}{crrrr}\mathbb{A}_{1} & \mathbb{A}_{3} & 3 \mathbb{A}_{1} & 3 \mathbb{A}_{1} & 5 \mathbb{A}_{1} \\
& 2 \mathbb{A}_{1} & \left(4 \mathbb{A}_{1}\right)_{I} & \left(4 \mathbb{A}_{1}\right)_{I} & 6 \mathbb{A}_{1} \\
& & 2 \mathbb{A}_{1} & \left(4 \mathbb{A}_{1}\right)_{I} & 6 \mathbb{A}_{1} \\
& & & 2 \mathbb{A}_{1} & 6 \mathbb{A}_{1} \\
& & & & 4 \mathbb{A}_{1}\end{array}\right)$ & 17 & $2_{6}^{+2}, 4_{2}^{-2}, 8_{7}^{+1}$ \\
\hline & & $\left(\begin{array}{rrrrr}\mathbb{A}_{1} & \mathbb{A}_{3} & 3 \mathbb{A}_{1} & 3 \mathbb{A}_{1} & 5 \mathbb{A}_{1} \\
& 2 \mathbb{A}_{1} & \left(4 \mathbb{A}_{1}\right)_{I I} & \left(4 \mathbb{A}_{1}\right)_{I} & 6 \mathbb{A}_{1} \\
& 2 \mathbb{A}_{1} & \left(4 \mathbb{A}_{1}\right)_{I} & 6 \mathbb{A}_{1} \\
& & 2 \mathbb{A}_{1} & 6 \mathbb{A}_{1} \\
& & & & 4 \mathbb{A}_{1}\end{array}\right) \subset \mathbb{A}_{3} \amalg 8 \mathbb{A}_{1}$ & 17 & $2_{I I}^{+2}, 4_{6}^{+2}, 8_{1}^{+1}$ \\
\hline & & $\left(\begin{array}{rrrrr}\mathbb{A}_{1} & \mathbb{A}_{3} & 3 \mathbb{A}_{1} & 3 \mathbb{A}_{1} & 5 \mathbb{A}_{1} \\
& 2 \mathbb{A}_{1} & \left(4 \mathbb{A}_{1}\right)_{I} & \left(4 \mathbb{A}_{1}\right)_{I} & 6 \mathbb{A}_{1} \\
& & 2 \mathbb{A}_{1} & 2 \mathbb{A}_{2} & 6 \mathbb{A}_{1} \\
& & & 2 \mathbb{A}_{1} & 6 \mathbb{A}_{1} \\
& & & & 4 \mathbb{A}_{1}\end{array}\right)$ & 17 & $2_{6}^{+4}, 8_{7}^{+1}, 3^{-1}$ \\
\hline
\end{tabular}




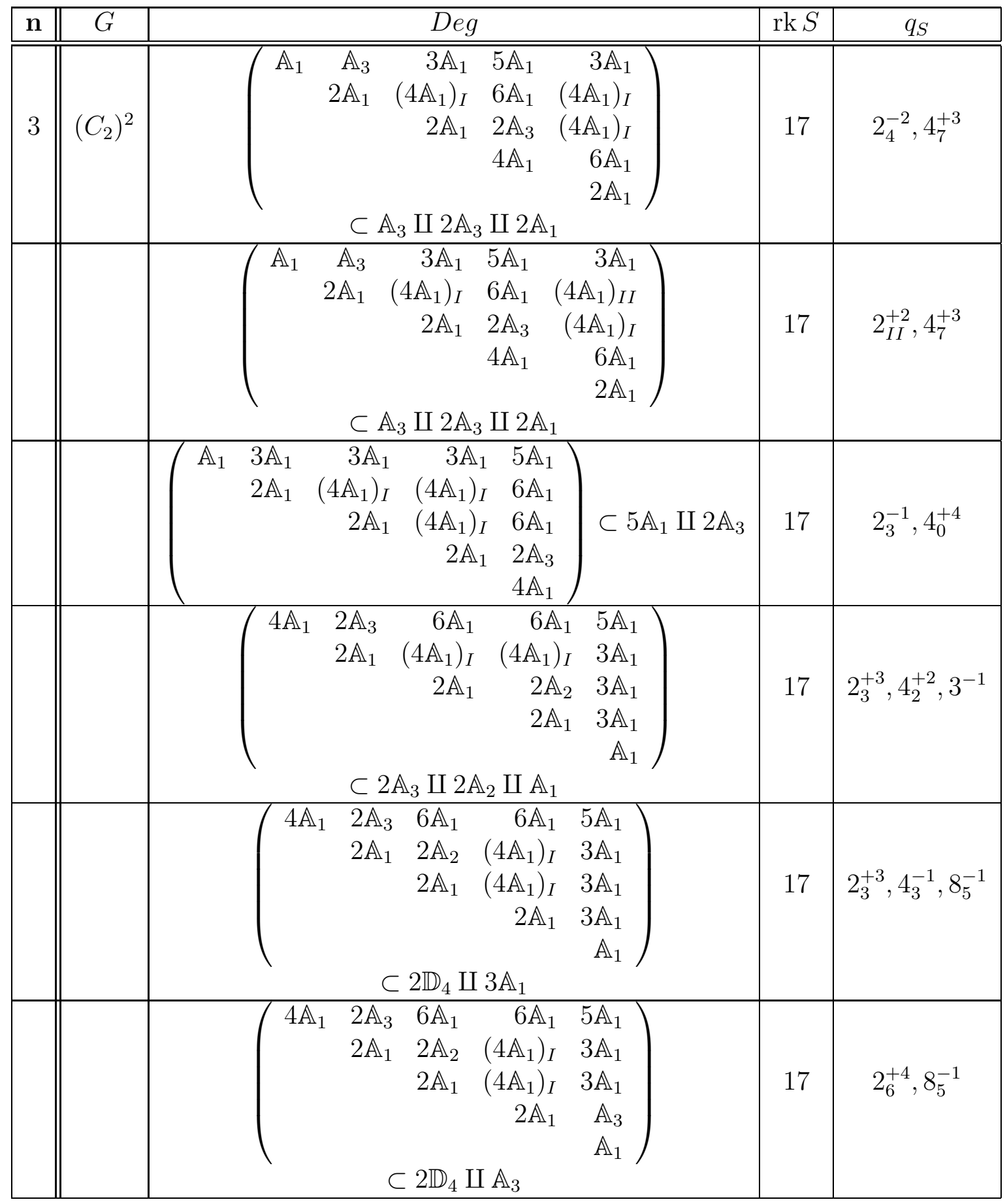




\begin{tabular}{|c|c|c|c|c|}
\hline $\mathbf{n}$ & $G$ & Deg & $\operatorname{rk} S$ & $q_{S}$ \\
\hline \multirow[t]{8}{*}{3} & $\left(C_{2}\right)^{2}$ & $\left.\begin{array}{rrrrr}4 \mathbb{A}_{1} & 6 \mathbb{A}_{1} & 6 \mathbb{A}_{1} & 6 \mathbb{A}_{1} & 5 \mathbb{A}_{1} \\
& 2 \mathbb{A}_{1} & \left(4 \mathbb{A}_{1}\right)_{I} & \left(4 \mathbb{A}_{1}\right)_{I} & 3 \mathbb{A}_{1} \\
& & 2 \mathbb{A}_{1} & 2 \mathbb{A}_{2} & 3 \mathbb{A}_{1} \\
& & & 2 \mathbb{A}_{1} & \mathbb{A}_{3} \\
& & & & \mathbb{A}_{1}\end{array}\right)$ & 17 & $2_{1}^{-3}, 4_{3}^{-1}, 8_{3}^{-1}$ \\
\hline & & $\left.\begin{array}{rrrrr}4 \mathbb{A}_{1} & 2 \mathbb{A}_{3} & 6 \mathbb{A}_{1} & 6 \mathbb{A}_{1} & 5 \mathbb{A}_{1} \\
& 2 \mathbb{A}_{1} & \left(4 \mathbb{A}_{1}\right)_{I} & \left(4 \mathbb{A}_{1}\right)_{I} & 3 \mathbb{A}_{1} \\
& & 2 \mathbb{A}_{1} & 2 \mathbb{A}_{2} & 3 \mathbb{A}_{1} \\
& & & 2 \mathbb{A}_{1} & \mathbb{A}_{3} \\
& & & & \mathbb{A}_{1}\end{array}\right)$ & 17 & $2_{1}^{-3}, 4_{2}^{+2}$ \\
\hline & & 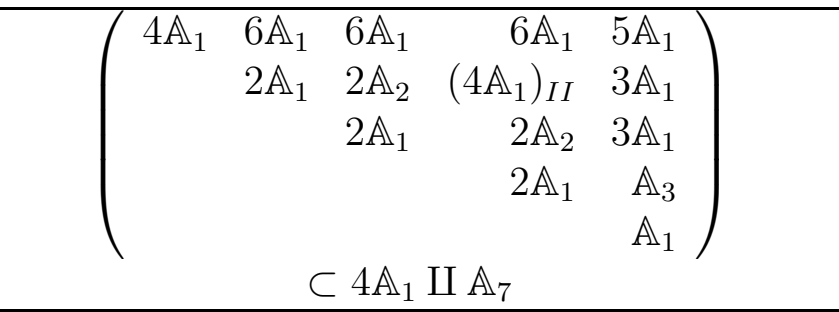 & 17 & $2_{I I}^{+4}, 8_{7}^{+1}$ \\
\hline & & $\left(\mathbb{A}_{1},\left(2 \mathbb{A}_{1}, 2 \mathbb{A}_{1}\right)_{I}, 4 \mathbb{A}_{1}, 4 \mathbb{A}_{1}\right) \subset 13 \mathbb{A}_{1}$ & 17 & $2_{5}^{-1}, 4_{6}^{-4}$ \\
\hline & & $\left(\mathbb{A}_{1},\left(2 \mathbb{A}_{1}, 2 \mathbb{A}_{1}\right)_{I}, 4 \mathbb{A}_{1}, 4 \mathbb{A}_{1}\right) \subset 5 \mathbb{A}_{1} \amalg 4 \mathbb{A}_{2}$ & 17 & $2_{5}^{+3}, 4_{4}^{-2}, 3^{+1}$ \\
\hline & & $\left(\mathbb{A}_{1}, 2 \mathbb{A}_{1}, 2 \mathbb{A}_{1}, 4 \mathbb{A}_{1}, 4 \mathbb{A}_{1}\right) \subset \mathbb{A}_{1} \amalg 2 \mathbb{A}_{2} \amalg 4 \mathbb{A}_{2}$ & 17 & $2_{3}^{-5}, 3^{-2}$ \\
\hline & & $\left(\begin{array}{rrrrr}\mathbb{A}_{1} & 3 \mathbb{A}_{1} & 5 \mathbb{A}_{1} & 5 \mathbb{A}_{1} & 5 \mathbb{A}_{1} \\
& 2 \mathbb{A}_{1} & 6 \mathbb{A}_{1} & \left(4 \mathbb{A}_{1}\right)_{I} & 6 \mathbb{A}_{1} \\
& & 4 \mathbb{A}_{1} & 6 \mathbb{A}_{1} & 8 \mathbb{A}_{1} \\
& & & 2 \mathbb{A}_{1} & 2 \mathbb{A}_{3} \\
& & & & 4 \mathbb{A}_{1}\end{array}\right) \subset 7 \mathbb{A}_{1} \amalg 2 A$ & 17 & $2_{5}^{-3}, 4_{7}^{+1}, 8_{7}^{+1}$ \\
\hline & & $\left(\begin{array}{rrrrr}2 \mathbb{A}_{1} & 3 \mathbb{A}_{1} & \left(4 \mathbb{A}_{1}\right)_{I} & 6 \mathbb{A}_{1} & 5 \mathbb{A}_{1} \\
& \mathbb{A}_{1} & \mathbb{A}_{3} & 5 \mathbb{A}_{1} & 5 \mathbb{A}_{1} \\
& & 2 \mathbb{A}_{1} & 6 \mathbb{A}_{1} & 6 \mathbb{A}_{1} \\
& & & 4 \mathbb{A}_{1} & 8 \mathbb{A}_{1} \\
& & & & 4 \mathbb{A}_{1}\end{array}\right)$ & 17 & $2_{4}^{-2}, 4_{7}^{+3}$ \\
\hline
\end{tabular}




\begin{tabular}{|c|c|c|c|c|}
\hline $\mathbf{n}$ & $G$ & Deg & rk $S$ & $q_{S}$ \\
\hline \multirow[t]{6}{*}{3} & $\left(C_{2}\right)^{2}$ & $\left(\begin{array}{rrrrr}2 \mathbb{A}_{1} & 3 \mathbb{A}_{1} & \left(4 \mathbb{A}_{1}\right)_{I} & 6 \mathbb{A}_{1} & 5 \mathbb{A}_{1} \\
& \mathbb{A}_{1} & \mathbb{A}_{3} & 5 \mathbb{A}_{1} & 5 \mathbb{A}_{1} \\
& & 2 \mathbb{A}_{1} & 6 \mathbb{A}_{1} & 6 \mathbb{A}_{1} \\
& & & 4 \mathbb{A}_{1} & 4 \mathbb{A}_{2} \\
& & & & 4 \mathbb{A}_{1}\end{array}\right)$ & 17 & $2_{6}^{+4}, 4_{3}^{-1}, 3^{+1}$ \\
\hline & & 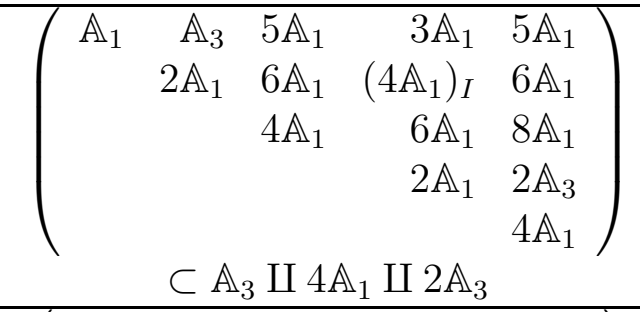 & 17 & $2_{6}^{+4}, 8_{5}^{-1}$ \\
\hline & & $\left.\begin{array}{crrrr}\mathbb{A}_{1} & \mathbb{A}_{3} & 3 \mathbb{A}_{1} & 5 \mathbb{A}_{1} & 5 \mathbb{A}_{1} \\
& 2 \mathbb{A}_{1} & \left(4 \mathbb{A}_{1}\right)_{I I} & 6 \mathbb{A}_{1} & 6 \mathbb{A}_{1} \\
& & 2 \mathbb{A}_{1} & 6 \mathbb{A}_{1} & 6 \mathbb{A}_{1} \\
& & & 4 \mathbb{A}_{1} & 8 \mathbb{A}_{1} \\
& & & & 4 \mathbb{A}_{1}\end{array}\right)$ & 17 & $2_{I I}^{+2}, 4_{7}^{+3}$ \\
\hline & & 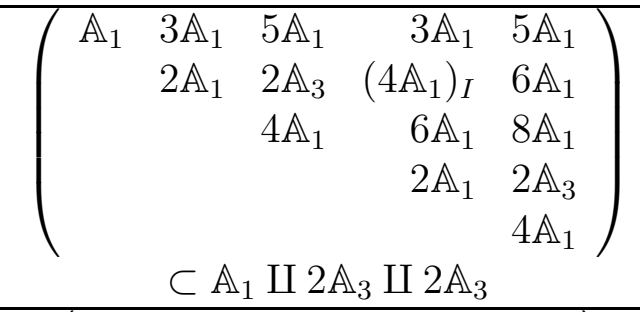 & 17 & $2_{3}^{-3}, 4_{I I}^{-2}$ \\
\hline & & $\left.\begin{array}{ccccc}4 \mathbb{A}_{1} & 2 \mathbb{A}_{3} & 6 \mathbb{A}_{1} & 8 \mathbb{A}_{1} & 5 \mathbb{A}_{1} \\
& 2 \mathbb{A}_{1} & 2 \mathbb{A}_{2} & 6 \mathbb{A}_{1} & 3 \mathbb{A}_{1} \\
& & 2 \mathbb{A}_{1} & 6 \mathbb{A}_{1} & 3 \mathbb{A}_{1} \\
& & & 4 \mathbb{A}_{1} & 5 \mathbb{A}_{1} \\
& & & & \mathbb{A}_{1}\end{array}\right)$ & 17 & $2_{7}^{+3}, 4_{I I}^{+2}$ \\
\hline & & $\left(4 \mathbb{A}_{1}, 4 \mathbb{A}_{1}, 2 \mathbb{A}_{1}, 2 \mathbb{A}_{1}, \mathbb{A}_{1}\right) \subset 4 \mathbb{A}_{2} \amalg \mathbb{A}_{5}$ & 17 & $22_{5}^{-5}, 3^{+1}$ \\
\hline
\end{tabular}




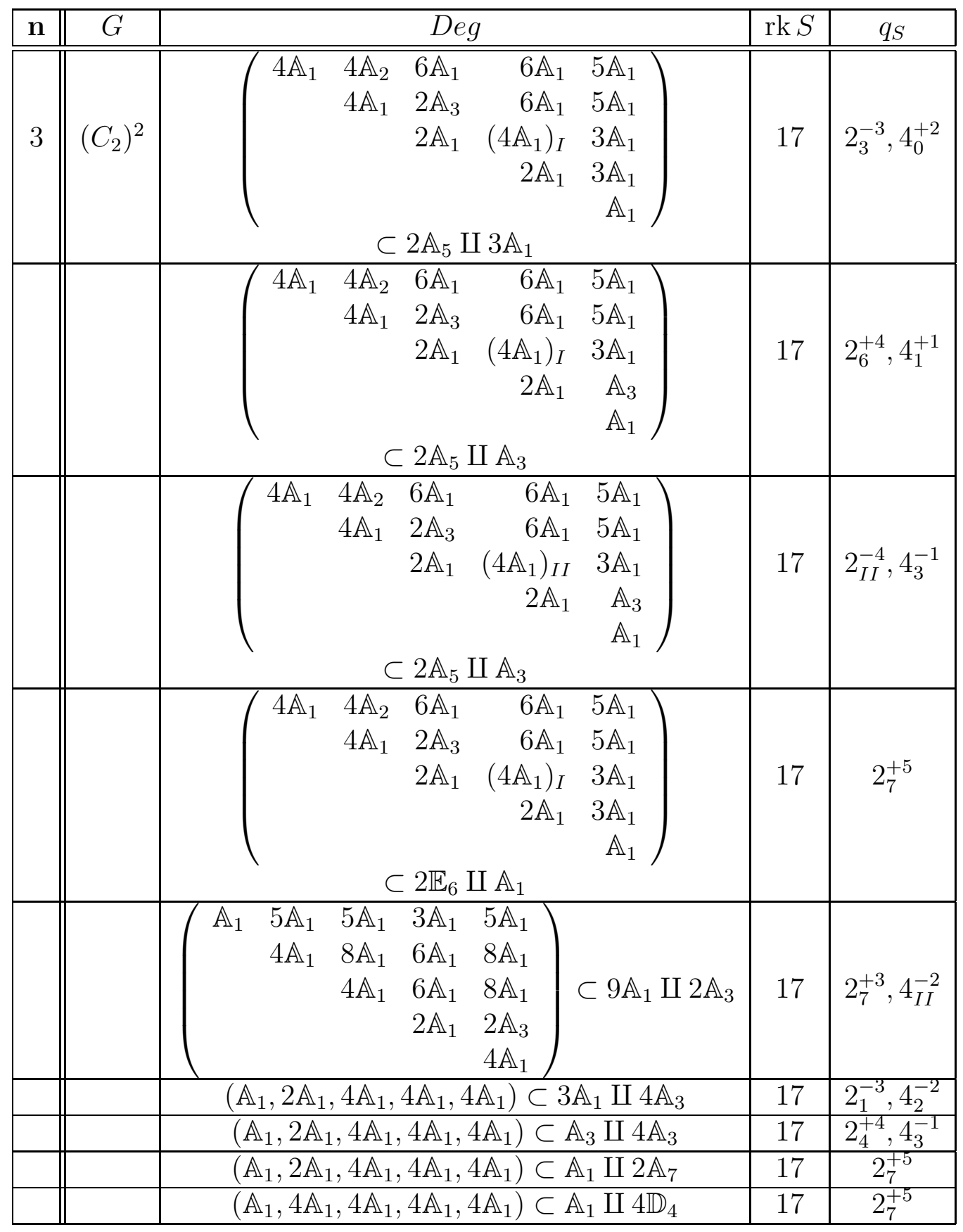




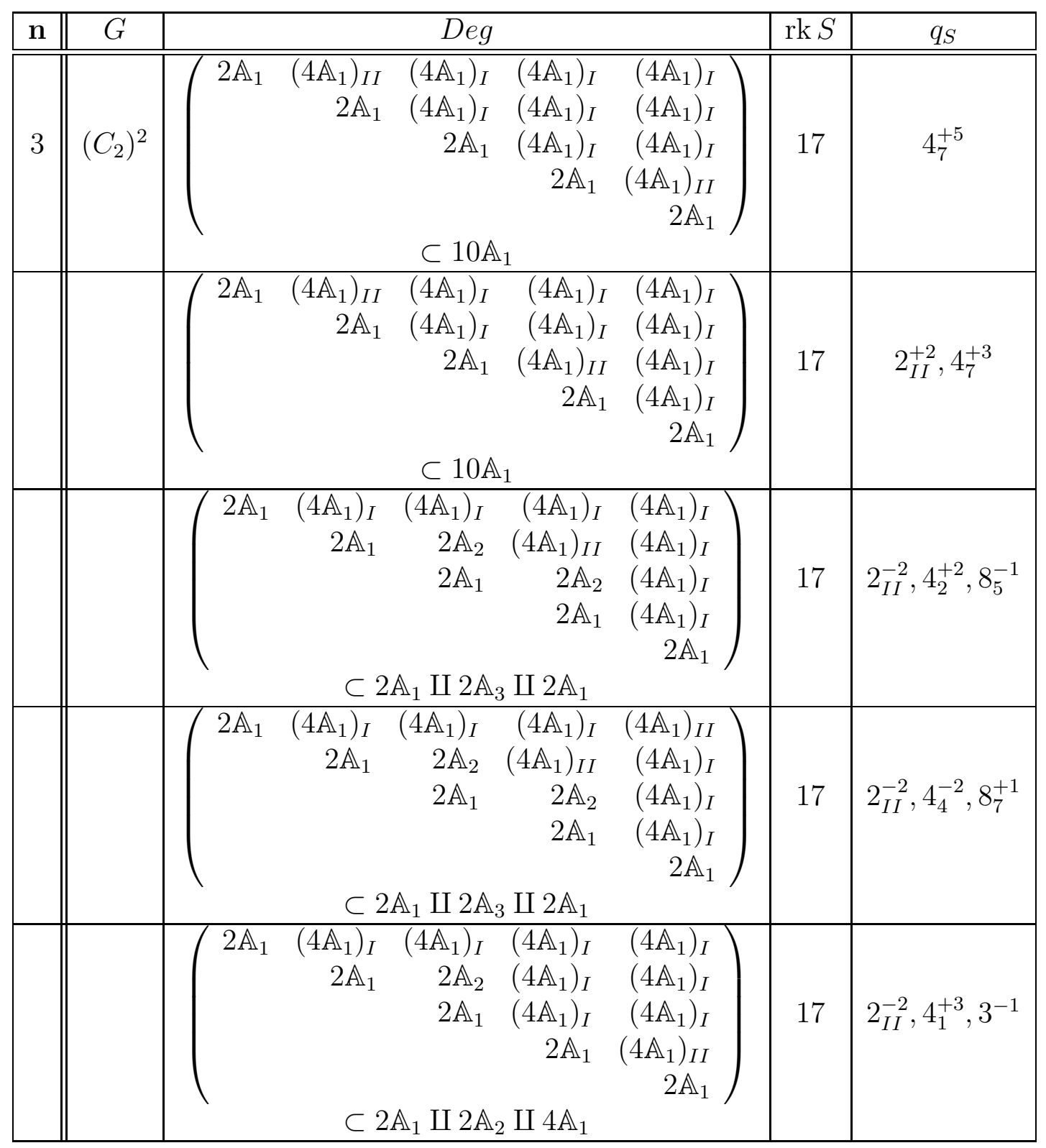




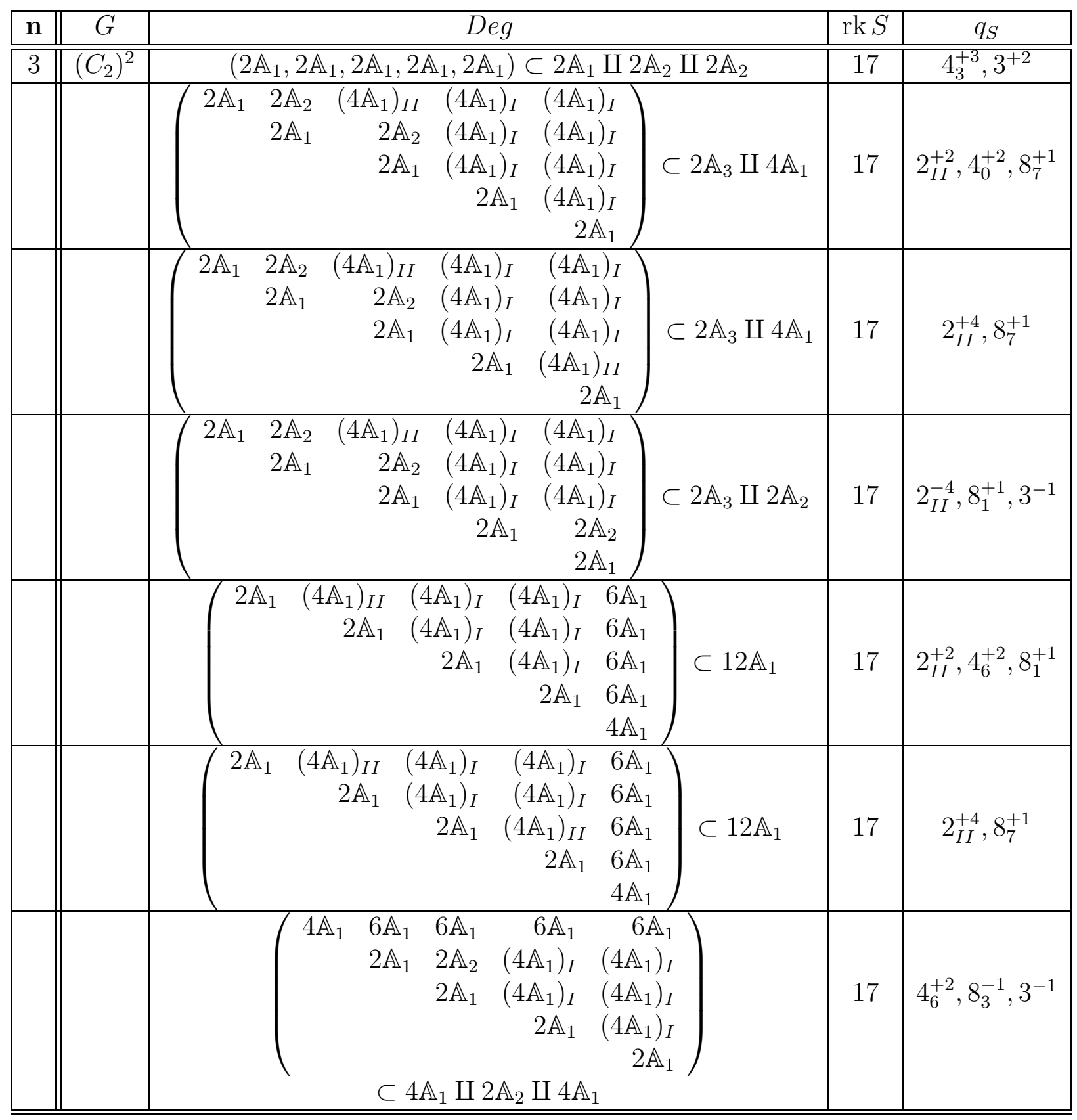




\begin{tabular}{|c|c|c|c|c|c|c|}
\hline $\mathbf{n}$ & $\bar{G}$ & \multicolumn{3}{|c|}{$D e g$} & rk $S$ & $q_{S}$ \\
\hline 3 & $\left(C_{2}\right)^{2}$ & & 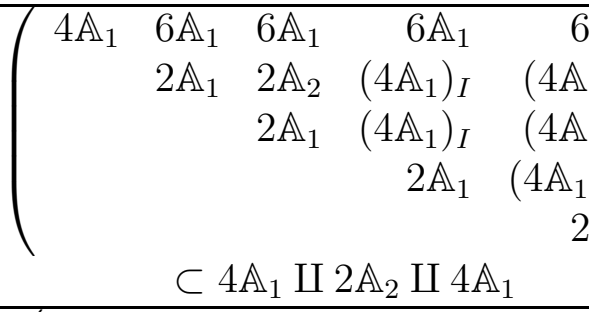 & $\begin{array}{l}\mathbb{A}_{1} \\
1)_{I} \\
1)_{I} \\
)_{I I} \\
\mathbb{A}_{1}\end{array}$ & 17 & $2_{I I}^{-4}, 8_{1}^{+1}, 3^{-1}$ \\
\hline & & & $\begin{array}{ccccc}4 \mathbb{A}_{1} & 6 \mathbb{A}_{1} & 6 \mathbb{A}_{1} & 6 \mathbb{A}_{1} & 6 \\
& 2 \mathbb{A}_{1} & 2 \mathbb{A}_{2} & \left(4 \mathbb{A}_{1}\right)_{I} & \left(4 \mathbb{A}_{1}\right. \\
& & 2 \mathbb{A}_{1} & \left(4 \mathbb{A}_{1}\right)_{I} & \left(4 \mathbb{A}_{1}\right. \\
& & & 2 \mathbb{A}_{1} & 2 \\
& & & & \\
& & \subset 4 \mathbb{A}_{1} \amalg 2 \mathbb{A}_{2} \amalg 2 \mathbb{A}_{2} \\
\end{array}$ & $\left.\begin{array}{l}\mathbb{A}_{1} \\
1)_{I} \\
)_{I} \\
\mathbb{A}_{2} \\
\mathbb{A}_{1}\end{array}\right)$ & 17 & $2_{I I}^{-2}, 8_{3}^{-1}, 3^{+2}$ \\
\hline & & $4 \mathbb{A}_{1}$ & $\begin{array}{rrrr}2 \mathbb{A}_{3} & 6 \mathbb{A}_{1} & 6 \mathbb{A}_{1} & 6 \mathbb{A}_{1} \\
2 \mathbb{A}_{1} & \left(4 \mathbb{A}_{1}\right)_{I I} & \left(4 \mathbb{A}_{1}\right)_{I} & \left(4 \mathbb{A}_{1}\right)_{I} \\
& 2 \mathbb{A}_{1} & \left(4 \mathbb{A}_{1}\right)_{I} & \left(4 \mathbb{A}_{1}\right)_{I} \\
& & 2 \mathbb{A}_{1} & \left(4 \mathbb{A}_{1}\right)_{I} \\
& & & 2 \mathbb{A}_{1}\end{array}$ & $\subset 2 \mathbb{A}_{3} \amalg 6 \mathbb{A}_{1}$ & 17 & $2_{I I}^{-2}, 4_{3}^{-3}$ \\
\hline & & $4 \mathbb{A}_{1}$ & $\begin{array}{rrrr}2 \mathbb{A}_{3} & 6 \mathbb{A}_{1} & 6 \mathbb{A}_{1} & 6 \mathbb{A}_{1} \\
2 \mathbb{A}_{1} & \left(4 \mathbb{A}_{1}\right)_{I} & \left(4 \mathbb{A}_{1}\right)_{I} & \left(4 \mathbb{A}_{1}\right)_{I} \\
& 2 \mathbb{A}_{1} & \left(4 \mathbb{A}_{1}\right)_{I} & \left(4 \mathbb{A}_{1}\right)_{I} \\
& & 2 \mathbb{A}_{1} & \left(4 \mathbb{A}_{1}\right)_{I I} \\
& & & 2 \mathbb{A}_{1} \\
\end{array}$ & $\subset 2 \mathbb{A}_{3} \amalg 6 \mathbb{A}_{1}$ & 17 & $2_{I I}^{-2}, 4_{3}^{-3}$ \\
\hline & & $4 \mathbb{A}_{1}$ & $\left.\begin{array}{rrrr}2 \mathbb{A}_{3} & 6 \mathbb{A}_{1} & 6 \mathbb{A}_{1} & 6 \mathbb{A}_{1} \\
2 \mathbb{A}_{1} & \left(4 \mathbb{A}_{1}\right)_{I I} & \left(4 \mathbb{A}_{1}\right)_{I} & \left(4 \mathbb{A}_{1}\right)_{I} \\
& 2 \mathbb{A}_{1} & \left(4 \mathbb{A}_{1}\right)_{I} & \left(4 \mathbb{A}_{1}\right)_{I} \\
& & 2 \mathbb{A}_{1} & \left(4 \mathbb{A}_{1}\right)_{I I} \\
& & & 2 \mathbb{A}_{1}\end{array}\right)$ & $\subset 2 \mathbb{A}_{3} \amalg 6 \mathbb{A}_{1}$ & 17 & $2_{I I}^{+2}, 4_{7}^{+3}$ \\
\hline & & & 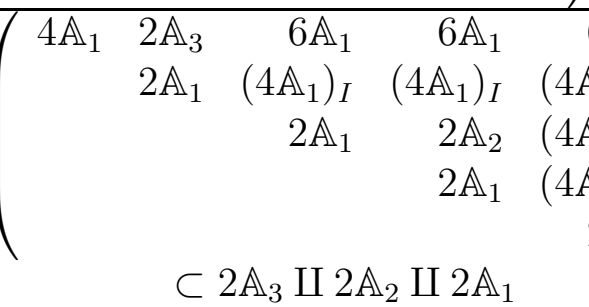 & $\left.\begin{array}{l}6 \mathbb{A}_{1} \\
\left.\mathbb{A}_{1}\right)_{I} \\
\left.\mathbb{A}_{1}\right)_{I} \\
\left.\mathbb{A}_{1}\right)_{I} \\
2 \mathbb{A}_{1}\end{array}\right)$ & 17 & $4_{5}^{-3}, 3^{-1}$ \\
\hline
\end{tabular}




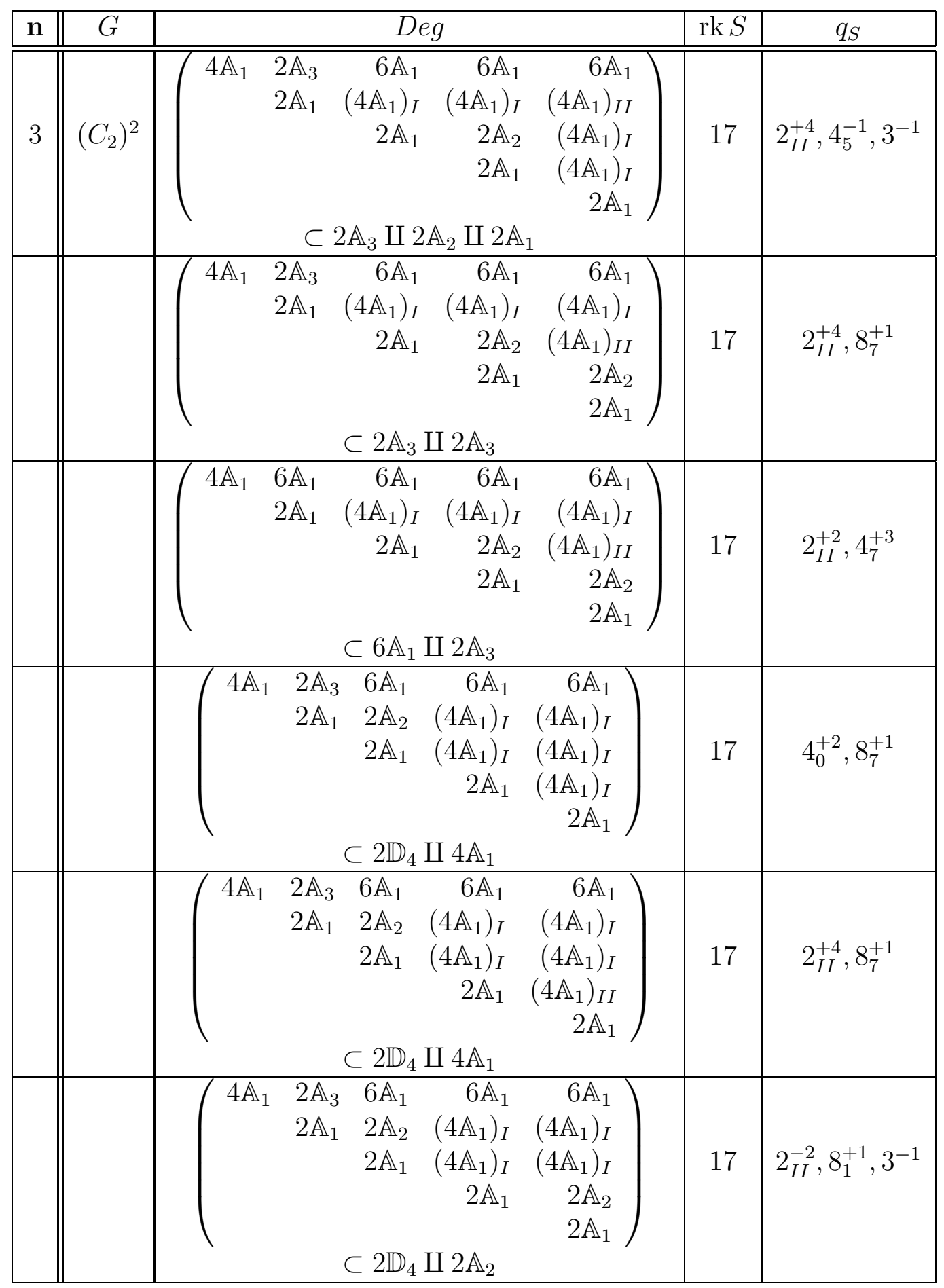




\begin{tabular}{|c|c|c|c|c|c|}
\hline $\mathbf{n}$ & $G$ & Deg & & rk $S$ & $q_{S}$ \\
\hline \multirow[t]{8}{*}{3} & $\left(C_{2}\right)^{2}$ & $\left.\begin{array}{cccrr}4 \mathbb{A}_{1} & 2 \mathbb{A}_{3} & 6 \mathbb{A}_{1} & 6 \mathbb{A}_{1} & 6 \mathbb{A}_{1} \\
& 2 \mathbb{A}_{1} & 2 \mathbb{A}_{2} & \left(4 \mathbb{A}_{1}\right)_{I I} & \left(4 \mathbb{A}_{1}\right)_{I} \\
& & 2 \mathbb{A}_{1} & 2 \mathbb{A}_{2} & \left(4 \mathbb{A}_{1}\right)_{I} \\
& & & 2 \mathbb{A}_{1} & \left(4 \mathbb{A}_{1}\right)_{I} \\
& & & & 2 \mathbb{A}_{1}\end{array}\right)$ & $\subset 2 \mathbb{D}_{5} \amalg 2 \mathbb{A}_{1}$ & 17 & $2_{I I}^{+4}, 4_{7}^{+1}$ \\
\hline & & $\left(\begin{array}{rrrrr}2 \mathbb{A}_{1} & \left(4 \mathbb{A}_{1}\right)_{I} & \left(4 \mathbb{A}_{1}\right)_{I} & 6 \mathbb{A}_{1} & 6 \mathbb{A}_{1} \\
& 2 \mathbb{A}_{1} & \left(4 \mathbb{A}_{1}\right)_{I} & 6 \mathbb{A}_{1} & 6 \mathbb{A}_{1} \\
& & 2 \mathbb{A}_{1} & 6 \mathbb{A}_{1} & 6 \mathbb{A}_{1} \\
& & & 4 \mathbb{A}_{1} & 8 \mathbb{A}_{1} \\
& & & & 4 \mathbb{A}_{1} \\
& & & \end{array}\right.$ & $\subset 14 \mathbb{A}_{1}$ & 17 & $2_{4}^{-2}, 4_{7}^{-3}$ \\
\hline & & $\left(\begin{array}{rrrrr}2 \mathbb{A}_{1} & \left(4 \mathbb{A}_{1}\right)_{I I} & \left(4 \mathbb{A}_{1}\right)_{I} & 6 \mathbb{A}_{1} & 6 \mathbb{A}_{1} \\
& 2 \mathbb{A}_{1} & \left(4 \mathbb{A}_{1}\right)_{I} & 6 \mathbb{A}_{1} & 6 \mathbb{A}_{1} \\
& & 2 \mathbb{A}_{1} & 6 \mathbb{A}_{1} & 6 \mathbb{A}_{1} \\
& & & 4 \mathbb{A}_{1} & 8 \mathbb{A}_{1} \\
& & & & 4 \mathbb{A}_{1} \\
& & & & \\
& & & \end{array}\right.$ & $\subset 14 \mathbb{A}_{1}$ & 17 & $2_{I I}^{+2}, 4_{7}^{+3}$ \\
\hline & & $\left(\begin{array}{rrrrr}2 \mathbb{A}_{1} & \left(4 \mathbb{A}_{1}\right)_{I} & \left(4 \mathbb{A}_{1}\right)_{I} & 6 \mathbb{A}_{1} & 6 \mathbb{A}_{1} \\
& 2 \mathbb{A}_{1} & \left(4 \mathbb{A}_{1}\right)_{I} & 6 \mathbb{A}_{1} & 6 \mathbb{A}_{1} \\
& & 2 \mathbb{A}_{1} & 6 \mathbb{A}_{1} & 6 \mathbb{A}_{1} \\
& & & 4 \mathbb{A}_{1} & 4 \mathbb{A}_{2} \\
& & & & 4 \mathbb{A}_{1}\end{array}\right)$ & $\subset 6 \mathbb{A}_{1} \amalg 4 \mathbb{A}_{2}$ & 17 & $4_{1}^{-3}, 3^{+1}$ \\
\hline & & $\left(2 \mathbb{A}_{1}, 2 \mathbb{A}_{1}, 2 \mathbb{A}_{1}, 4 \mathbb{A}_{1}, 4 \mathbb{A}_{1}\right) \subset 2 \mathbb{A}_{1} \amalg$ & $2 \mathbb{A}_{2} \amalg 4 \mathbb{A}_{2}$ & 17 & $2_{I I}^{+2}, 4_{7}^{+1}, 3^{-2}$ \\
\hline & & $\left(\begin{array}{rrrrr}2 \mathbb{A}_{1} & \left(4 \mathbb{A}_{1}\right)_{I} & 6 \mathbb{A}_{1} & \left(4 \mathbb{A}_{1}\right)_{I} & 6 \mathbb{A}_{1} \\
& 2 \mathbb{A}_{1} & 6 \mathbb{A}_{1} & \left(4 \mathbb{A}_{1}\right)_{I} & 6 \mathbb{A}_{1} \\
& & 4 \mathbb{A}_{1} & 6 \mathbb{A}_{1} & 8 \mathbb{A}_{1} \\
& & & 2 \mathbb{A}_{1} & 2 \mathbb{A}_{3} \\
& & & & 4 \mathbb{A}_{1}\end{array}\right)$ & $\subset 8 \mathbb{A}_{1} \amalg 2 \mathbb{A}_{3}$ & 17 & $4_{6}^{-2}, 8_{5}^{-1}$ \\
\hline & & $\left(\begin{array}{rrrrr}2 \mathbb{A}_{1} & \left(4 \mathbb{A}_{1}\right)_{I I} & 6 \mathbb{A}_{1} & \left(4 \mathbb{A}_{1}\right)_{I} & 6 \mathbb{A}_{1} \\
& 2 \mathbb{A}_{1} & 6 \mathbb{A}_{1} & \left(4 \mathbb{A}_{1}\right)_{I} & 6 \mathbb{A}_{1} \\
& & 4 \mathbb{A}_{1} & 6 \mathbb{A}_{1} & 8 \mathbb{A}_{1} \\
& & & 2 \mathbb{A}_{1} & 2 \mathbb{A}_{3} \\
& & & & 4 \mathbb{A}_{1}\end{array}\right)$ & $\subset 8 \mathbb{A}_{1} \amalg 2 \mathbb{A}_{3}$ & 17 & $2_{I I}^{+4}, 8_{7}^{+1}$ \\
\hline & & $\left(\begin{array}{rrrr}2 \mathbb{A}_{1} & \left(4 \mathbb{A}_{1}\right)_{I} & 6 \mathbb{A}_{1} & \left(4 \mathbb{A}_{1}\right)_{I} \\
& 2 \mathbb{A}_{1} & 2 \mathbb{A}_{3} & \left(4 \mathbb{A}_{1}\right)_{I} \\
& & 4 \mathbb{A}_{1} & 6 \mathbb{A}_{1} \\
& & & 2 \mathbb{A}_{1} \\
& & & \\
& \subset 2 \mathbb{A}_{1} \amalg 2 \mathbb{A}_{3} \amalg 2 \mathbb{A}_{3} \\
\end{array}\right.$ & $\begin{array}{l}6 \mathbb{A}_{1} \\
6 \mathbb{A}_{1} \\
8 \mathbb{A}_{1} \\
2 \mathbb{A}_{3} \\
4 \mathbb{A}_{1}\end{array}$ & 17 & $4_{7}^{+3}$ \\
\hline
\end{tabular}




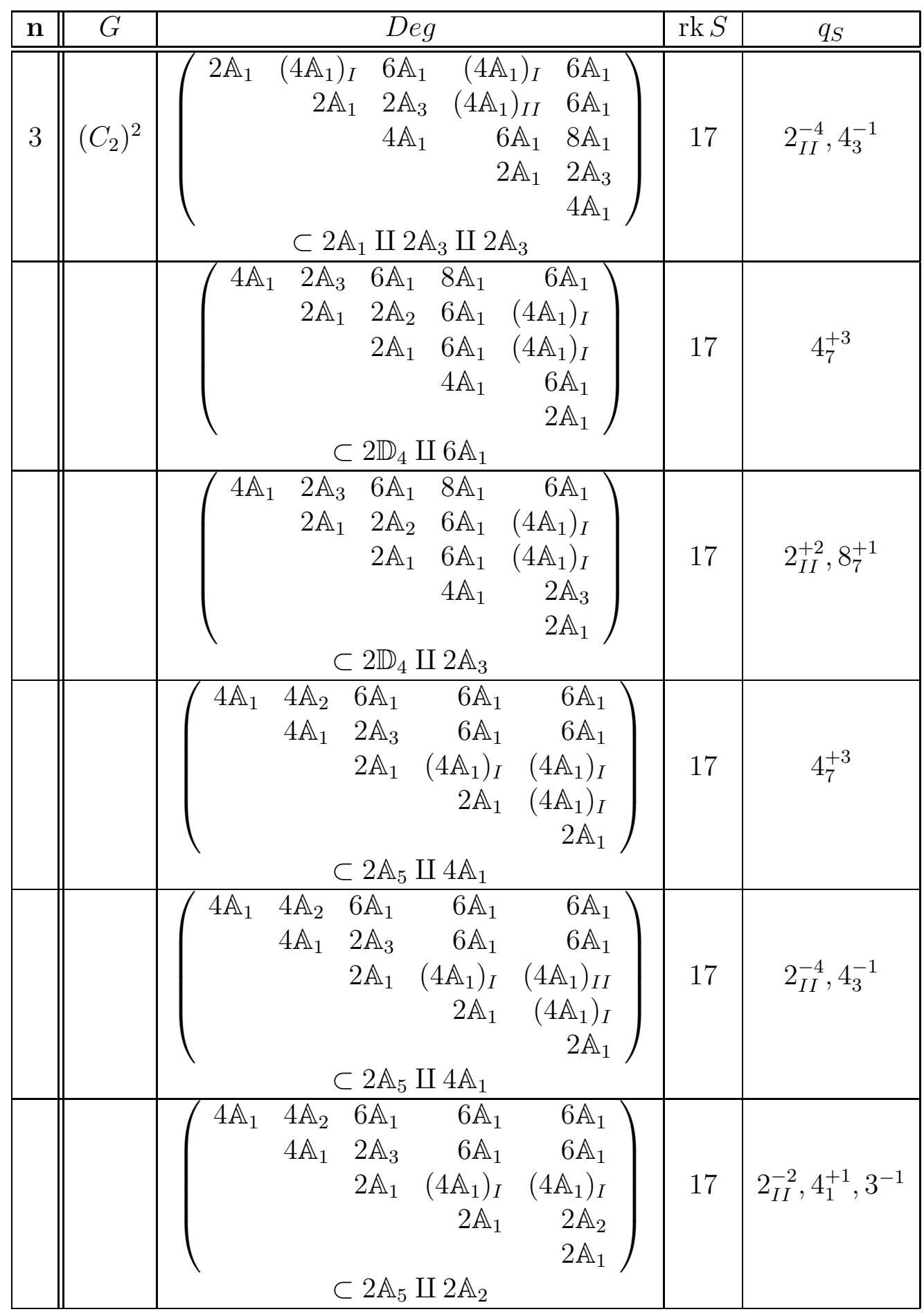




\begin{tabular}{|c|c|c|c|c|}
\hline n & $\bar{G}$ & $\overline{D e g}$ & $\operatorname{rk} S$ & $q_{S}$ \\
\hline 3 & $\left(C_{2}\right)^{2}$ & $\left.\begin{array}{ccccr}\mathbb{A}_{1} & 4 \mathbb{A}_{2} & 6 \mathbb{A}_{1} & 6 \mathbb{A}_{1} & 6 \mathbb{A}_{1} \\
& 4 \mathbb{A}_{1} & 2 \mathbb{A}_{3} & 6 \mathbb{A}_{1} & 6 \mathbb{A}_{1} \\
& & 2 \mathbb{A}_{1} & 2 \mathbb{A}_{2} & \left(4 \mathbb{A}_{1}\right)_{I} \\
& & & 2 \mathbb{A}_{1} & \left(4 \mathbb{A}_{1}\right)_{I} \\
& & & & 2 \mathbb{A}_{1}\end{array}\right)$ & 17 & $2_{I I}^{+2}, 4_{7}^{+1}$ \\
\hline & & $\left(\begin{array}{ccccr}4 \mathbb{A}_{1} & 4 \mathbb{A}_{2} & 8 \mathbb{A}_{1} & 6 \mathbb{A}_{1} & 6 \mathbb{A}_{1} \\
& 4 \mathbb{A}_{1} & 4 \mathbb{A}_{2} & 6 \mathbb{A}_{1} & 6 \mathbb{A}_{1} \\
& & 4 \mathbb{A}_{1} & 2 \mathbb{A}_{3} & 6 \mathbb{A}_{1} \\
& & & 2 \mathbb{A}_{1} & \left(4 \mathbb{A}_{1}\right)_{I} \\
& & & & 2 \mathbb{A}_{1}\end{array}\right)$ & 17 & $2_{I I}^{-2}, 4_{3}^{-1}$ \\
\hline & & 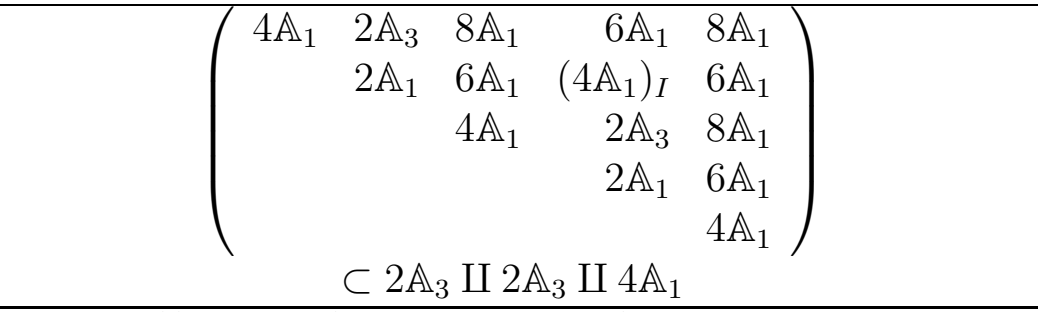 & 17 & $2_{I I}^{+2}, 8_{7}^{+1}$ \\
\hline & & $\left(\left(2 \mathbb{A}_{1}, 2 \mathbb{A}_{1}\right)_{I}, 4 \mathbb{A}_{1}, 4 \mathbb{A}_{1}, 4 \mathbb{A}_{1}\right) \subset 4 \mathbb{A}_{1} \amalg 4 \mathbb{A}_{3}$ & 17 & $4_{7}^{+3}$ \\
\hline & & $\left(\mathbb{A}_{1}, \mathbb{A}_{1}, \mathbb{A}_{1}, \mathbb{A}_{1}, 4 \mathbb{A}_{1}, 4 \mathbb{A}_{1}\right) \subset 12 \mathbb{A}_{1}$ & 18 & $2_{I I}^{+2}, 4_{6}^{+2}$ \\
\hline & & $\left(\begin{array}{rrrrrr}\mathbb{A}_{1} & \mathbb{A}_{3} & 2 \mathbb{A}_{1} & 3 \mathbb{A}_{1} & 2 \mathbb{A}_{1} & 3 \mathbb{A}_{1} \\
& 2 \mathbb{A}_{1} & 3 \mathbb{A}_{1} & \left(4 \mathbb{A}_{1}\right)_{I} & 3 \mathbb{A}_{1} & \left(4 \mathbb{A}_{1}\right)_{I} \\
& & \mathbb{A}_{1} & \mathbb{A}_{3} & 2 \mathbb{A}_{1} & 3 \mathbb{A}_{1} \\
& & & 2 \mathbb{A}_{1} & 3 \mathbb{A}_{1} & \left(4 \mathbb{A}_{1}\right)_{I} \\
& & & & \mathbb{A}_{1} & \mathbb{A}_{3} \\
& & & & & 2 \mathbb{A}_{1}\end{array}\right) \subset \mathbb{A}_{3} \amalg \mathbb{A}_{3} \amalg \mathbb{A}_{3}$ & 18 & $2_{6}^{+2}, 4_{I I}^{-2}$ \\
\hline & & $\left(\mathbb{A}_{1}, \mathbb{A}_{1},\left(2 \mathbb{A}_{1}, 2 \mathbb{A}_{1}\right)_{I}, \mathbb{A}_{1}, 4 \mathbb{A}_{1}\right) \subset \mathbb{A}_{1} \amalg \mathbb{A}_{3} \amalg \mathbb{A}_{3} \amalg 4 \mathbb{A}_{1}$ & 18 & $2_{3}^{-3}, 8_{3}^{-1}$ \\
\hline & & $\left(\mathbb{A}_{1}, \mathbb{A}_{1}, \mathbb{A}_{1}, 2 \mathbb{A}_{1}, 4 \mathbb{A}_{1}, 4 \mathbb{A}_{1}\right) \subset 2 \mathbb{A}_{1} \amalg \mathbb{A}_{3} \amalg 8 \mathbb{A}_{1}$ & 18 & $2_{0}^{+2}, 4_{6}^{+2}$ \\
\hline & & $\left(\mathbb{A}_{1}, \mathbb{A}_{1}, \mathbb{A}_{1}, 2 \mathbb{A}_{1}, 4 \mathbb{A}_{1}, 4 \mathbb{A}_{1}\right) \subset 2 \mathbb{A}_{1} \amalg \mathbb{A}_{3} \amalg 4 \mathbb{A}_{2}$ & 18 & $2_{4}^{-4}, 3^{+1}$ \\
\hline & & $\left(\mathbb{A}_{1}, \mathbb{A}_{1}, \mathbb{A}_{1}, 4 \mathbb{A}_{1}, 4 \mathbb{A}_{1}, 4 \mathbb{A}_{1}\right) \subset 15 \mathbb{A}_{1}$ & 18 & $2_{1}^{-3}, 8_{5}^{-1}$ \\
\hline & & $\left(4 \mathbb{A}_{1}, 4 \mathbb{A}_{1}, 4 \mathbb{A}_{1}, \mathbb{A}_{1}, \mathbb{A}_{1}, \mathbb{A}_{1}\right) \subset 4 \mathbb{A}_{3} \amalg 3 \mathbb{A}_{1}$ & 18 & $2_{5}^{+3}, 4_{1}^{+1}$ \\
\hline
\end{tabular}




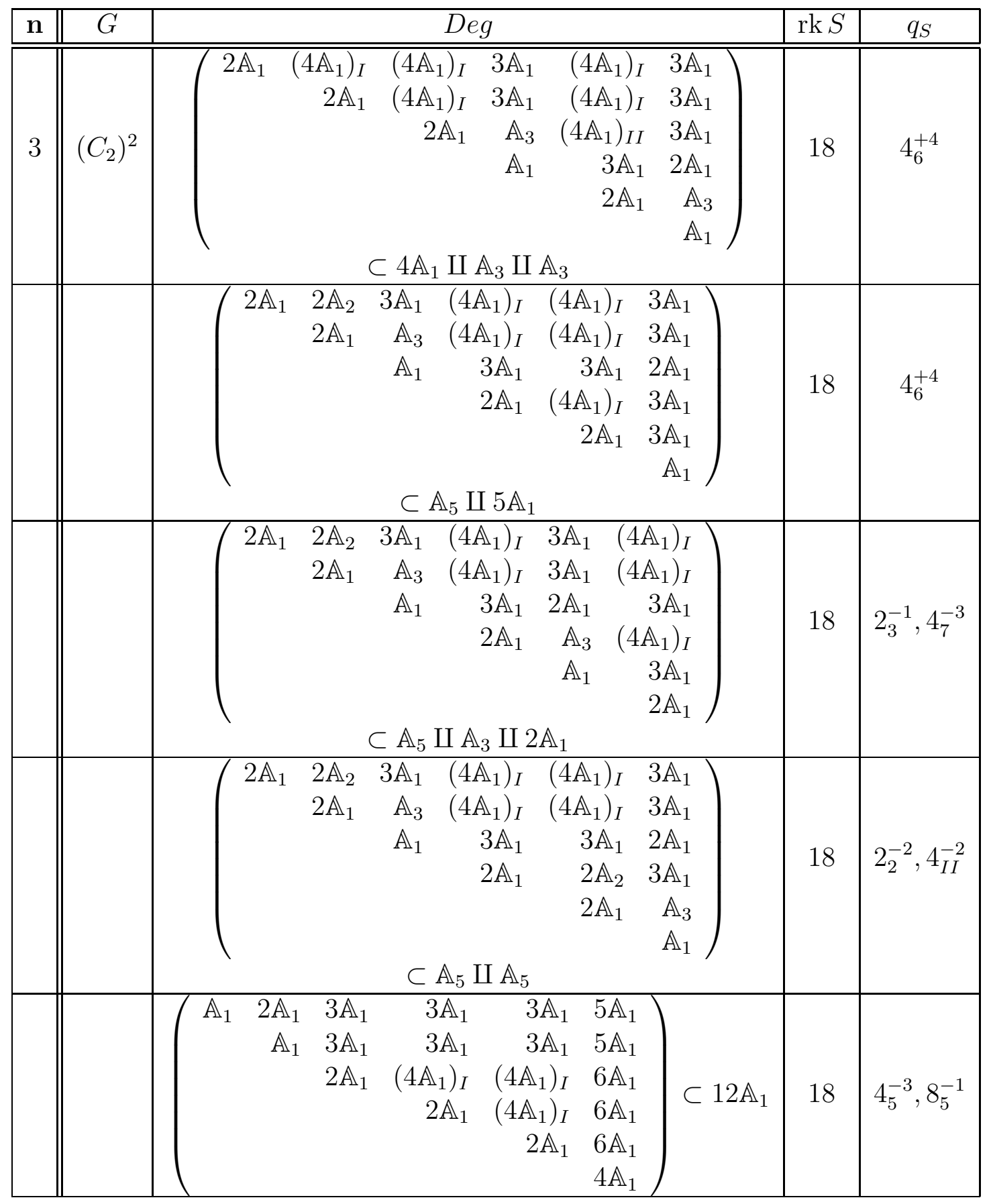




\begin{tabular}{|c|c|c|c|c|}
\hline n & $G$ & $D e g$ & rk $S$ & $q_{S}$ \\
\hline \multirow[t]{5}{*}{3} & $\left(C_{2}\right)^{2}$ & $\left.\begin{array}{rrrrrr}\mathbb{A}_{1} & 2 \mathbb{A}_{1} & 3 \mathbb{A}_{1} & 3 \mathbb{A}_{1} & 3 \mathbb{A}_{1} & 5 \mathbb{A}_{1} \\
& \mathbb{A}_{1} & \mathbb{A}_{3} & 3 \mathbb{A}_{1} & 3 \mathbb{A}_{1} & 5 \mathbb{A}_{1} \\
& & 2 \mathbb{A}_{1} & \left(4 \mathbb{A}_{1}\right)_{I} & \left(4 \mathbb{A}_{1}\right)_{I} & 6 \mathbb{A}_{1} \\
& & & 2 \mathbb{A}_{1} & \left(4 \mathbb{A}_{1}\right)_{I} & 6 \mathbb{A}_{1} \\
& & & & 2 \mathbb{A}_{1} & 2 \mathbb{A}_{3} \\
& & & & & 4 \mathbb{A}_{1}\end{array}\right)$ & 18 & $2_{7}^{+1}, 4_{7}^{+3}$ \\
\hline & & $\left(\begin{array}{crrrrr}\mathbb{A}_{1} & 3 \mathbb{A}_{1} & 3 \mathbb{A}_{1} & 3 \mathbb{A}_{1} & 2 \mathbb{A}_{1} & 5 \mathbb{A}_{1} \\
& 2 \mathbb{A}_{1} & \left(4 \mathbb{A}_{1}\right)_{I} & \left(4 \mathbb{A}_{1}\right)_{I} & 3 \mathbb{A}_{1} & 6 \mathbb{A}_{1} \\
& & 2 \mathbb{A}_{1} & \left(4 \mathbb{A}_{1}\right)_{I} & 3 \mathbb{A}_{1} & 6 \mathbb{A}_{1} \\
& & & 2 \mathbb{A}_{1} & \mathbb{A}_{3} & 6 \mathbb{A}_{1} \\
& & & & \mathbb{A}_{1} & 5 \mathbb{A}_{1} \\
& & & & & 4 \mathbb{A}_{1}\end{array}\right)$ & 18 & $2_{5}^{-1}, 4_{6}^{+2}, 8_{7}^{+1}$ \\
\hline & & $\left(\begin{array}{rrrrrr}4 \mathbb{A}_{1} & 6 \mathbb{A}_{1} & 5 \mathbb{A}_{1} & 6 \mathbb{A}_{1} & 5 \mathbb{A}_{1} & 6 \mathbb{A}_{1} \\
& 2 \mathbb{A}_{1} & \mathbb{A}_{3} & \left(4 \mathbb{A}_{1}\right)_{I} & 3 \mathbb{A}_{1} & \left(4 \mathbb{A}_{1}\right)_{I} \\
& & \mathbb{A}_{1} & 3 \mathbb{A}_{1} & 2 \mathbb{A}_{1} & 3 \mathbb{A}_{1} \\
& & & 2 \mathbb{A}_{1} & \mathbb{A}_{3} & \left(4 \mathbb{A}_{1}\right)_{I} \\
& & & & \mathbb{A}_{1} & 3 \mathbb{A}_{1} \\
& & & & & 2 \mathbb{A}_{1}\end{array}\right)$ & 18 & $2_{6}^{-2}, 4_{5}^{-1}, 8_{3}^{-1}$ \\
\hline & & $\left(\begin{array}{crrrrr}4 \mathbb{A}_{1} & 6 \mathbb{A}_{1} & 6 \mathbb{A}_{1} & 5 \mathbb{A}_{1} & 6 \mathbb{A}_{1} & 5 \mathbb{A}_{1} \\
& 2 \mathbb{A}_{1} & \left(4 \mathbb{A}_{1}\right)_{I} & 3 \mathbb{A}_{1} & \left(4 \mathbb{A}_{1}\right)_{I} & 3 \mathbb{A}_{1} \\
& & 2 \mathbb{A}_{1} & \mathbb{A}_{3} & \left(4 \mathbb{A}_{1}\right)_{I I} & 3 \mathbb{A}_{1} \\
& & & \mathbb{A}_{1} & 3 \mathbb{A}_{1} & 2 \mathbb{A}_{1} \\
& & & & 2 \mathbb{A}_{1} & \mathbb{A}_{3} \\
& & & & & \mathbb{A}_{1}\end{array}\right)$ & 18 & $2_{I I}^{+2}, 4_{5}^{-1}, 8_{5}^{-1}$ \\
\hline & & $\left(\begin{array}{rrrrrr}4 \mathbb{A}_{1} & 2 \mathbb{A}_{3} & 6 \mathbb{A}_{1} & 5 \mathbb{A}_{1} & 6 \mathbb{A}_{1} & 5 \mathbb{A}_{1} \\
& 2 \mathbb{A}_{1} & \left(4 \mathbb{A}_{1}\right)_{I} & 3 \mathbb{A}_{1} & \left(4 \mathbb{A}_{1}\right)_{I} & 3 \mathbb{A}_{1} \\
& & 2 \mathbb{A}_{1} & \mathbb{A}_{3} & \left(4 \mathbb{A}_{1}\right)_{I} & 3 \mathbb{A}_{1} \\
& & & \mathbb{A}_{1} & 3 \mathbb{A}_{1} & 2 \mathbb{A}_{1} \\
& & & & 2 \mathbb{A}_{1} & \mathbb{A}_{3} \\
& & & & & \mathbb{A}_{1}\end{array}\right)$ & 18 & $2_{6}^{+2}, 4_{0}^{+2}$ \\
\hline
\end{tabular}




\begin{tabular}{|c|c|c|c|c|}
\hline n & $\bar{G}$ & $\overline{D e g}$ & $\operatorname{rks} S$ & $q_{S}$ \\
\hline \multirow[t]{6}{*}{3} & $\left(C_{2}\right)^{2}$ & $\left.\begin{array}{crrrrr}4 \mathbb{A}_{1} & 2 \mathbb{A}_{3} & 6 \mathbb{A}_{1} & 5 \mathbb{A}_{1} & 6 \mathbb{A}_{1} & 5 \mathbb{A}_{1} \\
& 2 \mathbb{A}_{1} & \left(4 \mathbb{A}_{1}\right)_{I} & 3 \mathbb{A}_{1} & \left(4 \mathbb{A}_{1}\right)_{I} & 3 \mathbb{A}_{1} \\
& & 2 \mathbb{A}_{1} & \mathbb{A}_{3} & \left(4 \mathbb{A}_{1}\right)_{I I} & 3 \mathbb{A}_{1} \\
& & & \mathbb{A}_{1} & 3 \mathbb{A}_{1} & 2 \mathbb{A}_{1} \\
& & & & 2 \mathbb{A}_{1} & \mathbb{A}_{3} \\
& & & & & \mathbb{A}_{1}\end{array}\right)$ & 18 & $2_{I I}^{-2}, 4_{2}^{-2}$ \\
\hline & & $\left(\begin{array}{rrrrrr}4 \mathbb{A}_{1} & 6 \mathbb{A}_{1} & 6 \mathbb{A}_{1} & 6 \mathbb{A}_{1} & 5 \mathbb{A}_{1} & 5 \mathbb{A}_{1} \\
& 2 \mathbb{A}_{1} & \left(4 \mathbb{A}_{1}\right)_{I} & \left(4 \mathbb{A}_{1}\right)_{I} & 3 \mathbb{A}_{1} & 3 \mathbb{A}_{1} \\
& & 2 \mathbb{A}_{1} & 2 \mathbb{A}_{2} & 3 \mathbb{A}_{1} & 3 \mathbb{A}_{1} \\
& & & 2 \mathbb{A}_{1} & \mathbb{A}_{3} & 3 \mathbb{A}_{1} \\
& & & & \mathbb{A}_{1} & 2 \mathbb{A}_{1} \\
& & & & & \mathbb{A}_{1}\end{array}\right)$ & 18 & $2_{2}^{-2}, 4_{7}^{+1}, 8_{1}^{+1}$ \\
\hline & & $\left.\begin{array}{cccccc}4 \mathbb{A}_{1} & 6 \mathbb{A}_{1} & 6 \mathbb{A}_{1} & 5 \mathbb{A}_{1} & 6 \mathbb{A}_{1} & 5 \mathbb{A}_{1} \\
& 2 \mathbb{A}_{1} & 2 \mathbb{A}_{2} & 3 \mathbb{A}_{1} & \left(4 \mathbb{A}_{1}\right)_{I} & 3 \mathbb{A}_{1} \\
& & 2 \mathbb{A}_{1} & \mathbb{A}_{3} & \left(4 \mathbb{A}_{1}\right)_{I} & 3 \mathbb{A}_{1} \\
& & & \mathbb{A}_{1} & 3 \mathbb{A}_{1} & 2 \mathbb{A}_{1} \\
& & & & 2 \mathbb{A}_{1} & \mathbb{A}_{3} \\
& & & & & \mathbb{A}_{1}\end{array}\right)$ & 18 & $2_{7}^{+3}, 8_{7}^{+1}$ \\
\hline & & $\left(\begin{array}{rrrrrr}4 \mathbb{A}_{1} & 2 \mathbb{A}_{3} & 6 \mathbb{A}_{1} & 6 \mathbb{A}_{1} & 5 \mathbb{A}_{1} & 5 \mathbb{A}_{1} \\
& 2 \mathbb{A}_{1} & \left(4 \mathbb{A}_{1}\right)_{I} & \left(4 \mathbb{A}_{1}\right)_{I} & 3 \mathbb{A}_{1} & 3 \mathbb{A}_{1} \\
& & 2 \mathbb{A}_{1} & 2 \mathbb{A}_{2} & 3 \mathbb{A}_{1} & 3 \mathbb{A}_{1} \\
& & & 2 \mathbb{A}_{1} & \mathbb{A}_{3} & 3 \mathbb{A}_{1} \\
& & & & \mathbb{A}_{1} & 2 \mathbb{A}_{1} \\
& & & & & \mathbb{A}_{1}\end{array}\right)$ & 18 & $2_{6}^{+2}, 4_{0}^{+2}$ \\
\hline & & $\left(\mathbb{A}_{1}, \mathbb{A}_{1},\left(2 \mathbb{A}_{1}, 2 \mathbb{A}_{1}\right)_{I}, 4 \mathbb{A}_{1}, 4 \mathbb{A}_{1}\right) \subset 14 \mathbb{A}_{1}$ & 18 & $4_{6}^{+4}$ \\
\hline & & 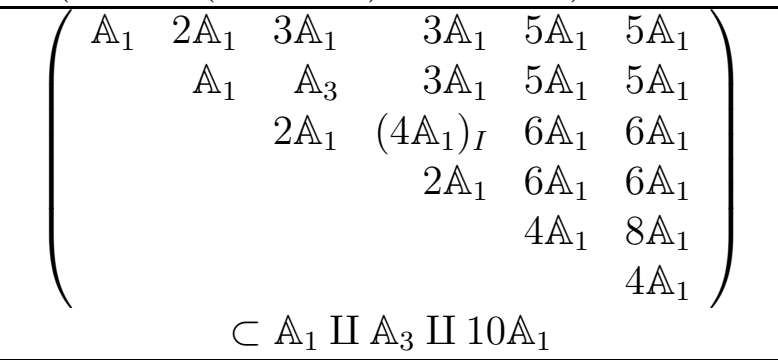 & 18 & $2_{5}^{-1}, 4_{5}^{+3}$ \\
\hline
\end{tabular}




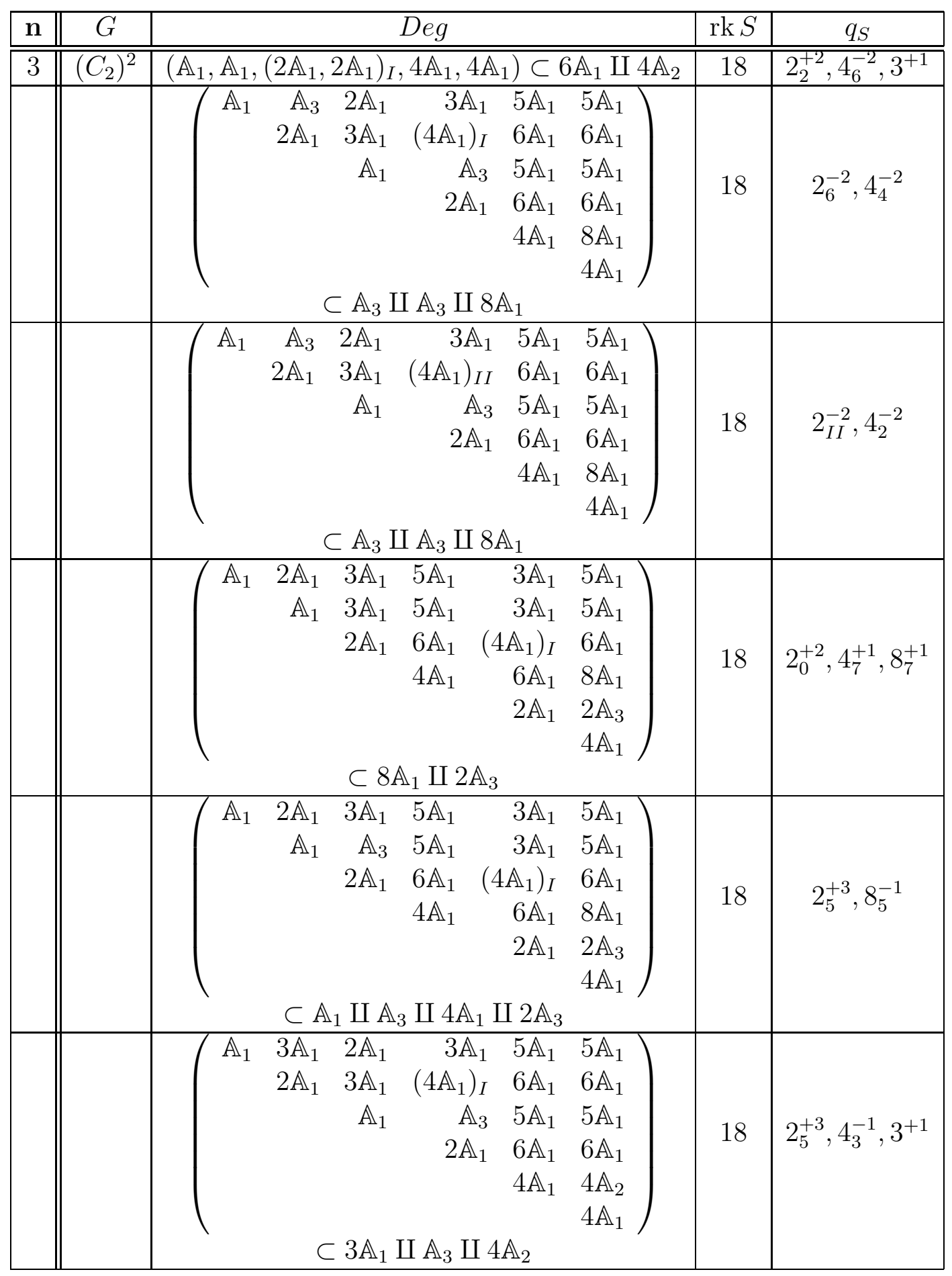




\begin{tabular}{|c|c|c|c|c|}
\hline n & $G$ & $\overline{D e g}$ & rk $S$ & $q_{S}$ \\
\hline 3 & $\left(C_{2}\right)^{2}$ & $\left.\begin{array}{rrrrrr}\mathbb{A}_{1} & \mathbb{A}_{3} & 2 \mathbb{A}_{1} & 3 \mathbb{A}_{1} & 5 \mathbb{A}_{1} & 5 \mathbb{A}_{1} \\
& 2 \mathbb{A}_{1} & 3 \mathbb{A}_{1} & \left(4 \mathbb{A}_{1}\right)_{I} & 6 \mathbb{A}_{1} & 6 \mathbb{A}_{1} \\
& & \mathbb{A}_{1} & \mathbb{A}_{3} & 5 \mathbb{A}_{1} & 5 \mathbb{A}_{1} \\
& & & 2 \mathbb{A}_{1} & 6 \mathbb{A}_{1} & 6 \mathbb{A}_{1} \\
& & & & 4 \mathbb{A}_{1} & 4 \mathbb{A}_{2} \\
& & & & & 4 \mathbb{A}_{1}\end{array}\right)$ & 18 & $2_{4}^{-4}, 3^{+1}$ \\
\hline & & $\left.\begin{array}{cccccc}4 \mathbb{A}_{1} & 4 \mathbb{A}_{2} & 6 \mathbb{A}_{1} & 6 \mathbb{A}_{1} & 5 \mathbb{A}_{1} & 5 \mathbb{A}_{1} \\
& 4 \mathbb{A}_{1} & 6 \mathbb{A}_{1} & 6 \mathbb{A}_{1} & 5 \mathbb{A}_{1} & 5 \mathbb{A}_{1} \\
& & 2 \mathbb{A}_{1} & 2 \mathbb{A}_{2} & 3 \mathbb{A}_{1} & 3 \mathbb{A}_{1} \\
& & & 2 \mathbb{A}_{1} & \mathbb{A}_{3} & 3 \mathbb{A}_{1} \\
& & & & \mathbb{A}_{1} & 2 \mathbb{A}_{1} \\
& & & & & \mathbb{A}_{1}\end{array}\right)$ & 18 & $2_{0}^{+4}, 3^{+1}$ \\
\hline & & $\left.\begin{array}{cccrcc}4 \mathbb{A}_{1} & 4 \mathbb{A}_{2} & 6 \mathbb{A}_{1} & 6 \mathbb{A}_{1} & 5 \mathbb{A}_{1} & 5 \mathbb{A}_{1} \\
& 4 \mathbb{A}_{1} & 2 \mathbb{A}_{3} & 6 \mathbb{A}_{1} & 5 \mathbb{A}_{1} & 5 \mathbb{A}_{1} \\
& & 2 \mathbb{A}_{1} & \left(4 \mathbb{A}_{1}\right)_{I} & 3 \mathbb{A}_{1} & 3 \mathbb{A}_{1} \\
& & & 2 \mathbb{A}_{1} & 3 \mathbb{A}_{1} & 3 \mathbb{A}_{1} \\
& & & & \mathbb{A}_{1} & 2 \mathbb{A}_{1} \\
& & & & & \mathbb{A}_{1}\end{array}\right)$ & 18 & $2_{2}^{+2}, 4_{4}^{-2}$ \\
\hline & & $\left.\begin{array}{cccccc}4 \mathbb{A}_{1} & 4 \mathbb{A}_{2} & 6 \mathbb{A}_{1} & 6 \mathbb{A}_{1} & 5 \mathbb{A}_{1} & 5 \mathbb{A}_{1} \\
& 4 \mathbb{A}_{1} & 2 \mathbb{A}_{3} & 6 \mathbb{A}_{1} & 5 \mathbb{A}_{1} & 5 \mathbb{A}_{1} \\
& & 2 \mathbb{A}_{1} & \left(4 \mathbb{A}_{1}\right)_{I} & 3 \mathbb{A}_{1} & 3 \mathbb{A}_{1} \\
& & & 2 \mathbb{A}_{1} & \mathbb{A}_{3} & 3 \mathbb{A}_{1} \\
& & & & \mathbb{A}_{1} & 2 \mathbb{A}_{1} \\
& & & & & \mathbb{A}_{1}\end{array}\right)$ & 18 & $2_{5}^{-3}, 4_{5}^{-1}$ \\
\hline & & $\left(4 \mathbb{A}_{1},\left(2 \mathbb{A}_{1}, 2 \mathbb{A}_{1}\right)_{I}, 4 \mathbb{A}_{1}, \mathbb{A}_{1}, \mathbb{A}_{1}\right) \subset 2 \mathbb{A}_{3} \amalg 2 \mathbb{A}_{3} \amalg 2 \mathbb{A}_{1}$ & 18 & $2_{2}^{-2}, 4_{I I}^{+2}$ \\
\hline & & $\left(4 \mathbb{A}_{1}, 2 \mathbb{A}_{1}, 4 \mathbb{A}_{1}, 4 \mathbb{A}_{1}, \mathbb{A}_{1}, \mathbb{A}_{1}\right) \subset 2 \mathbb{A}_{3} \amalg 10 \mathbb{A}_{1}$ & 18 & $2_{2}^{-2}, 4_{I I}^{-2}$ \\
\hline & & $\left(4 \mathbb{A}_{1}, 4 \mathbb{A}_{1}, 4 \mathbb{A}_{1}, 2 \mathbb{A}_{1}, \mathbb{A}_{1}, \mathbb{A}_{1}\right) \subset 4 \mathbb{A}_{3} \amalg 4 \mathbb{A}_{1}$ & 18 & $2_{I I}^{-2}, 4_{2}^{-2}$ \\
\hline & & $\left(4 \mathbb{A}_{1}, 4 \mathbb{A}_{1}, 4 \mathbb{A}_{1}, 2 \mathbb{A}_{1}, \mathbb{A}_{1}, \mathbb{A}_{1}\right) \subset 4 \mathbb{A}_{3} \amalg 4 \mathbb{A}_{1}$ & 18 & $2_{2}^{+2}, 4_{4}^{-2}$ \\
\hline & & $\left(4 \mathbb{A}_{1}, 4 \mathbb{A}_{1}, 4 \mathbb{A}_{1}, 2 \mathbb{A}_{1}, \mathbb{A}_{1}, \mathbb{A}_{1}\right) \subset 4 \mathbb{A}_{3} \amalg \mathbb{A}_{3} \amalg \mathbb{A}_{1}$ & 18 & $2_{7}^{-3}, 4_{3}^{-1}$ \\
\hline & & $\left(4 \mathbb{A}_{1}, 4 \mathbb{A}_{1}, 4 \mathbb{A}_{1}, 2 \mathbb{A}_{1}, \mathbb{A}_{1}, \mathbb{A}_{1}\right) \subset 2 \mathbb{A}_{7} \amalg 2 \mathbb{A}_{1}$ & 18 & $2_{6}^{+4}$ \\
\hline & & $\left(\mathbb{A}_{1}, \mathbb{A}_{1}, 4 \mathbb{A}_{1}, 4 \mathbb{A}_{1}, 4 \mathbb{A}_{1}, 4 \mathbb{A}_{1}\right) \subset 2 \mathbb{A}_{1} \amalg 4 \mathbb{D}_{4}$ & 18 & $2_{6}^{+4}$ \\
\hline & & $\left(\mathbb{A}_{1}, 2 \mathbb{A}_{1}, 2 \mathbb{A}_{1}, 2 \mathbb{A}_{1}, 2 \mathbb{A}_{1}, 2 \mathbb{A}_{1}\right) \subset 3 \mathbb{A}_{1} \amalg 2 \mathbb{A}_{2} \amalg 2 \mathbb{A}_{2}$ & 18 & $2_{1}^{+1}, 4_{1}^{-3}, 3^{+2}$ \\
\hline
\end{tabular}




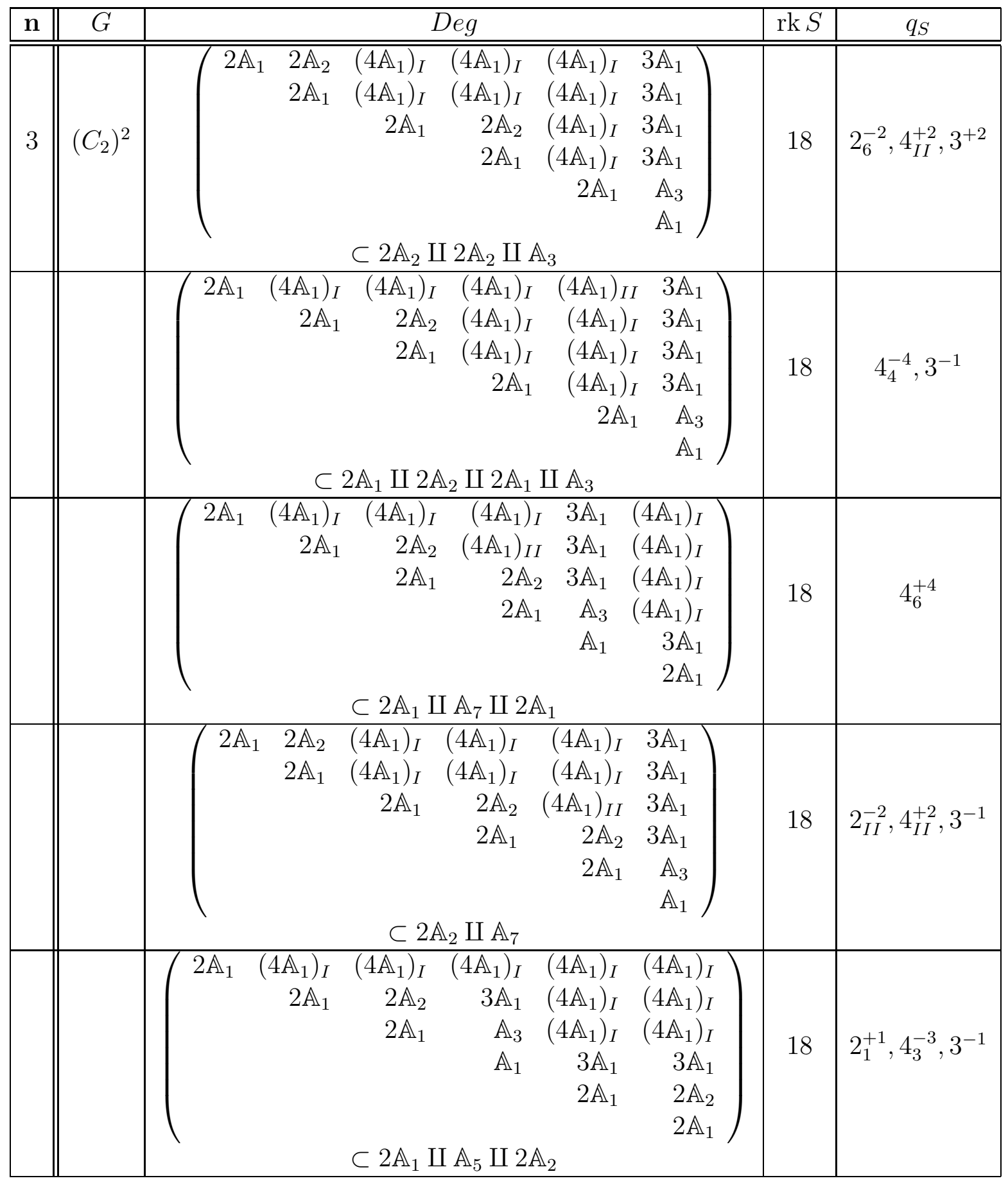




\begin{tabular}{|c|c|c|c|c|}
\hline $\mathbf{n}$ & $\bar{G}$ & $\overline{D e g}$ & $\operatorname{rk} S$ & $q_{S}$ \\
\hline \multirow[t]{6}{*}{3} & $\left(C_{2}\right)^{2}$ & $\left.\begin{array}{crrrrr}4 \mathbb{A}_{1} & 6 \mathbb{A}_{1} & 6 \mathbb{A}_{1} & 6 \mathbb{A}_{1} & 6 \mathbb{A}_{1} & 5 \mathbb{A}_{1} \\
& 2 \mathbb{A}_{1} & \left(4 \mathbb{A}_{1}\right)_{I} & \left(4 \mathbb{A}_{1}\right)_{I} & \left(4 \mathbb{A}_{1}\right)_{I} & 3 \mathbb{A}_{1} \\
& 2 \mathbb{A}_{1} & \left(4 \mathbb{A}_{1}\right)_{I} & \left(4 \mathbb{A}_{1}\right)_{I} & 3 \mathbb{A}_{1} \\
& & 2 \mathbb{A}_{1} & \left(4 \mathbb{A}_{1}\right)_{I I} & 3 \mathbb{A}_{1} \\
& & & 2 \mathbb{A}_{1} & \mathbb{A}_{3} \\
& & & & & \mathbb{A}_{1}\end{array}\right)$ & 18 & $4_{5}^{-3}, 8_{5}^{-1}$ \\
\hline & & $\begin{array}{c}\left(\mathbb{A}_{1},\left(2 \mathbb{A}_{1}, 2 \mathbb{A}_{1}\right)_{I}, 2 \mathbb{A}_{1}, 2 \mathbb{A}_{1}, 4 \mathbb{A}_{1}\right) \\
\subset 5 \mathbb{A}_{1} \amalg 2 \mathbb{A}_{2} \amalg 4 \mathbb{A}_{1}\end{array}$ & 18 & $2_{3}^{-1}, 4_{4}^{-2}, 8_{5}^{-1}, 3^{-1}$ \\
\hline & & $\left.\begin{array}{cccrrr}\mathbb{A}_{1} & 6 \mathbb{A}_{1} & 6 \mathbb{A}_{1} & 6 \mathbb{A}_{1} & 6 \mathbb{A}_{1} & 5 \mathbb{A}_{1} \\
& 2 \mathbb{A}_{1} & 2 \mathbb{A}_{2} & \left(4 \mathbb{A}_{1}\right)_{I} & \left(4 \mathbb{A}_{1}\right)_{I} & 3 \mathbb{A}_{1} \\
& & 2 \mathbb{A}_{1} & \left(4 \mathbb{A}_{1}\right)_{I} & \left(4 \mathbb{A}_{1}\right)_{I} & 3 \mathbb{A}_{1} \\
& & & 2 \mathbb{A}_{1} & \left(4 \mathbb{A}_{1}\right)_{I} & 3 \mathbb{A}_{1} \\
& & & & 2 \mathbb{A}_{1} & \mathbb{A}_{3} \\
& & & & & \mathbb{A}_{1}\end{array}\right)$ & 18 & $2_{6}^{-2}, 4_{3}^{-1}, 8_{7}^{+1}, 3^{-1}$ \\
\hline & & $\left.\begin{array}{cccrrr}4 \mathbb{A}_{1} & 6 \mathbb{A}_{1} & 6 \mathbb{A}_{1} & 6 \mathbb{A}_{1} & 6 \mathbb{A}_{1} & 5 \mathbb{A}_{1} \\
& 2 \mathbb{A}_{1} & 2 \mathbb{A}_{2} & \left(4 \mathbb{A}_{1}\right)_{I} & \left(4 \mathbb{A}_{1}\right)_{I} & 3 \mathbb{A}_{1} \\
& & 2 \mathbb{A}_{1} & \left(4 \mathbb{A}_{1}\right)_{I} & \left(4 \mathbb{A}_{1}\right)_{I} & 3 \mathbb{A}_{1} \\
& & & 2 \mathbb{A}_{1} & \left(4 \mathbb{A}_{1}\right)_{I I} & 3 \mathbb{A}_{1} \\
& & & & 2 \mathbb{A}_{1} & \mathbb{A}_{3} \\
& & & & & \mathbb{A}_{1}\end{array}\right)$ & 18 & $2_{I I}^{-2}, 4_{1}^{+1}, 8_{7}^{+1}, 3^{-1}$ \\
\hline & & $\begin{array}{c}\left(\mathbb{A}_{1}, 2 \mathbb{A}_{1}, 2 \mathbb{A}_{1}, 2 \mathbb{A}_{1}, 2 \mathbb{A}_{1}, 4 \mathbb{A}_{1}\right) \\
\subset \mathbb{A}_{1} \amalg 2 \mathbb{A}_{2} \amalg 2 \mathbb{A}_{2} \amalg 4 \mathbb{A}_{1}\end{array}$ & 18 & $2_{3}^{+3}, 8_{3}^{-1}, 3^{+2}$ \\
\hline & & $\left(\begin{array}{rrrrrr}\mathbb{A}_{1} & \mathbb{A}_{3} & 3 \mathbb{A}_{1} & 3 \mathbb{A}_{1} & 3 \mathbb{A}_{1} & 5 \mathbb{A}_{1} \\
& 2 \mathbb{A}_{1} & \left(4 \mathbb{A}_{1}\right)_{I I} & \left(4 \mathbb{A}_{1}\right)_{I} & \left(4 \mathbb{A}_{1}\right)_{I} & 6 \mathbb{A}_{1} \\
& & 2 \mathbb{A}_{1} & \left(4 \mathbb{A}_{1}\right)_{I} & \left(4 \mathbb{A}_{1}\right)_{I} & 6 \mathbb{A}_{1} \\
& & 2 \mathbb{A}_{1} & \left(4 \mathbb{A}_{1}\right)_{I} & 6 \mathbb{A}_{1} \\
& & & & 2 \mathbb{A}_{1} & 2 \mathbb{A}_{3} \\
& & & & & 4 \mathbb{A}_{1}\end{array}\right)$ & 18 & $4_{6}^{+4}$ \\
\hline
\end{tabular}




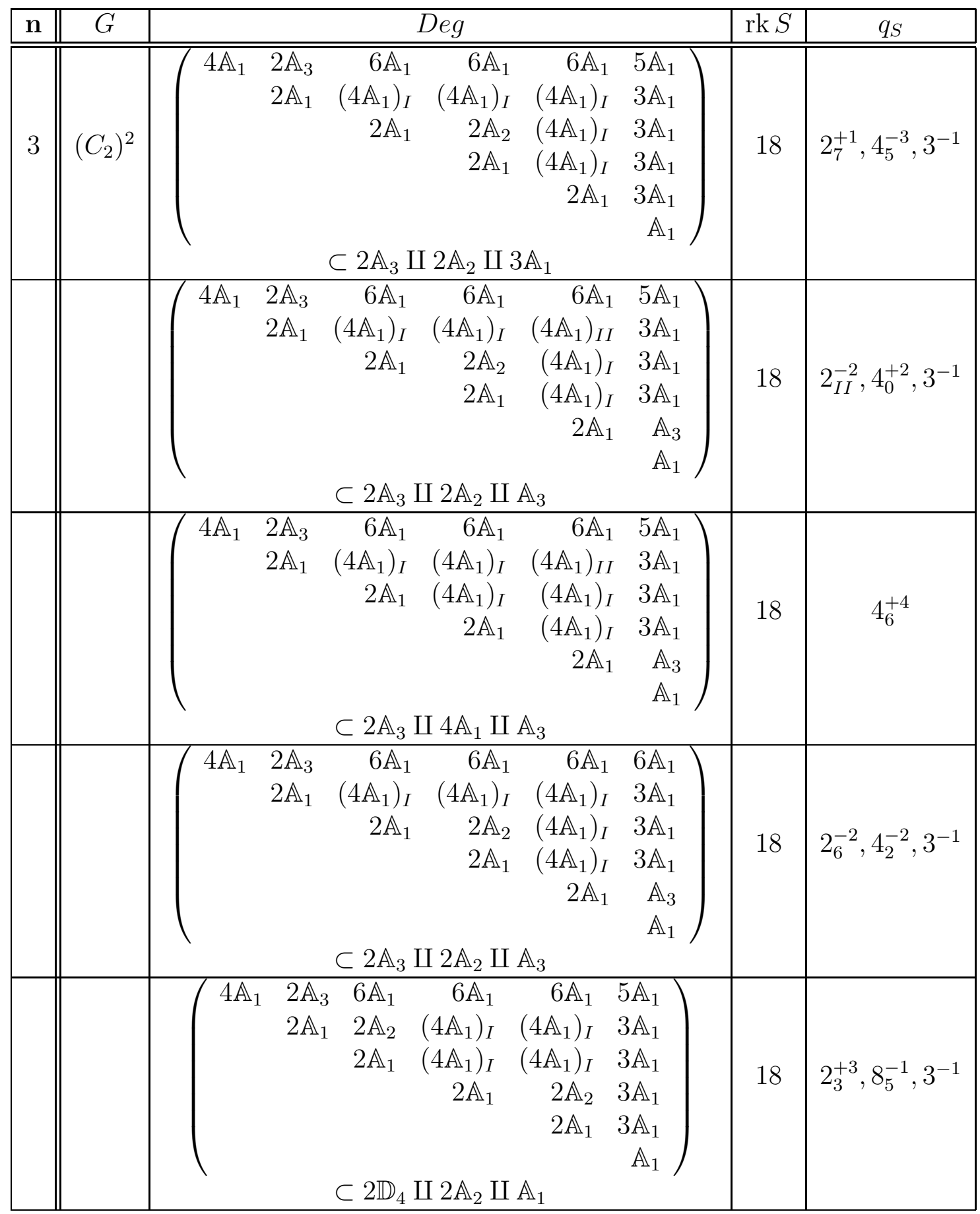




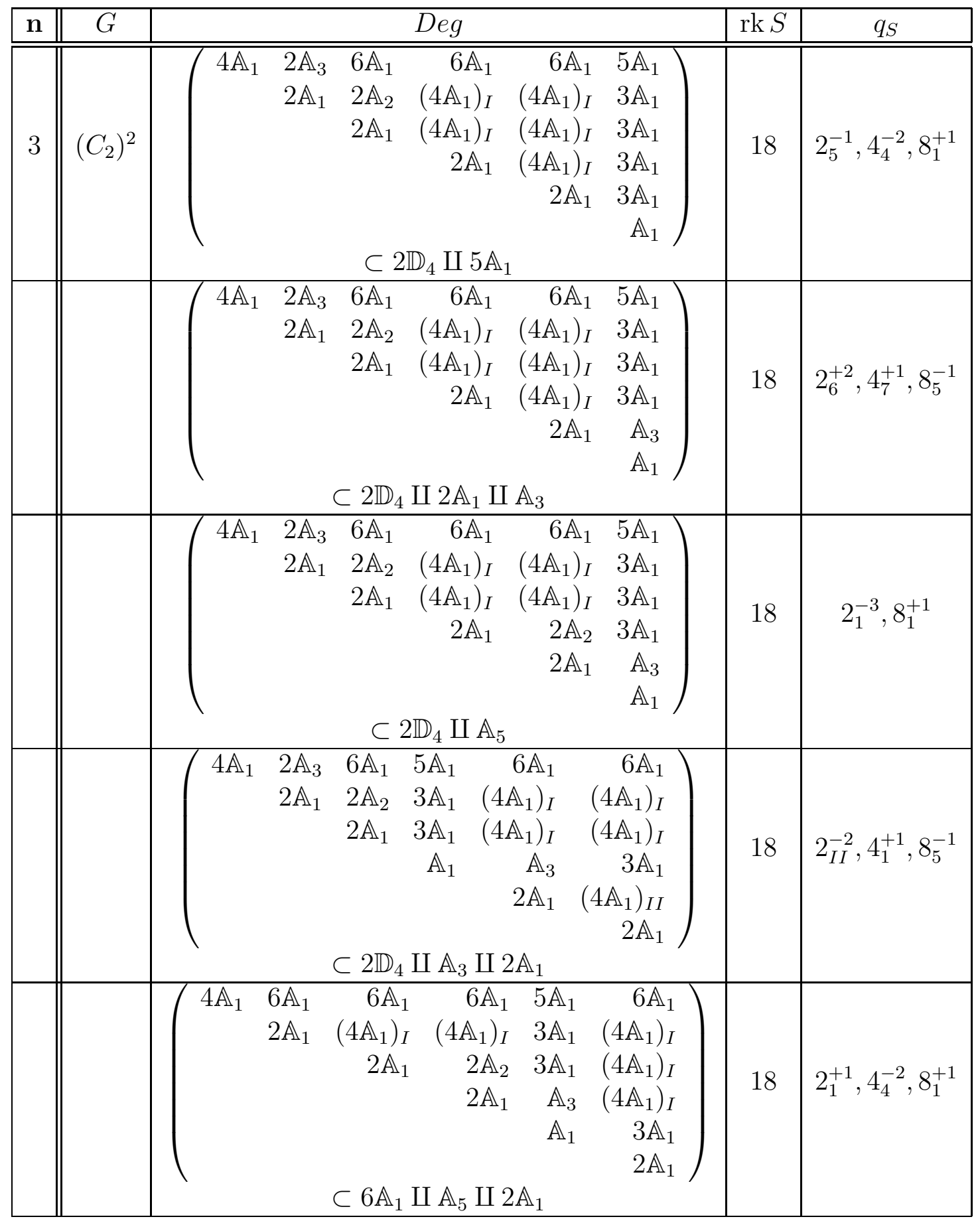




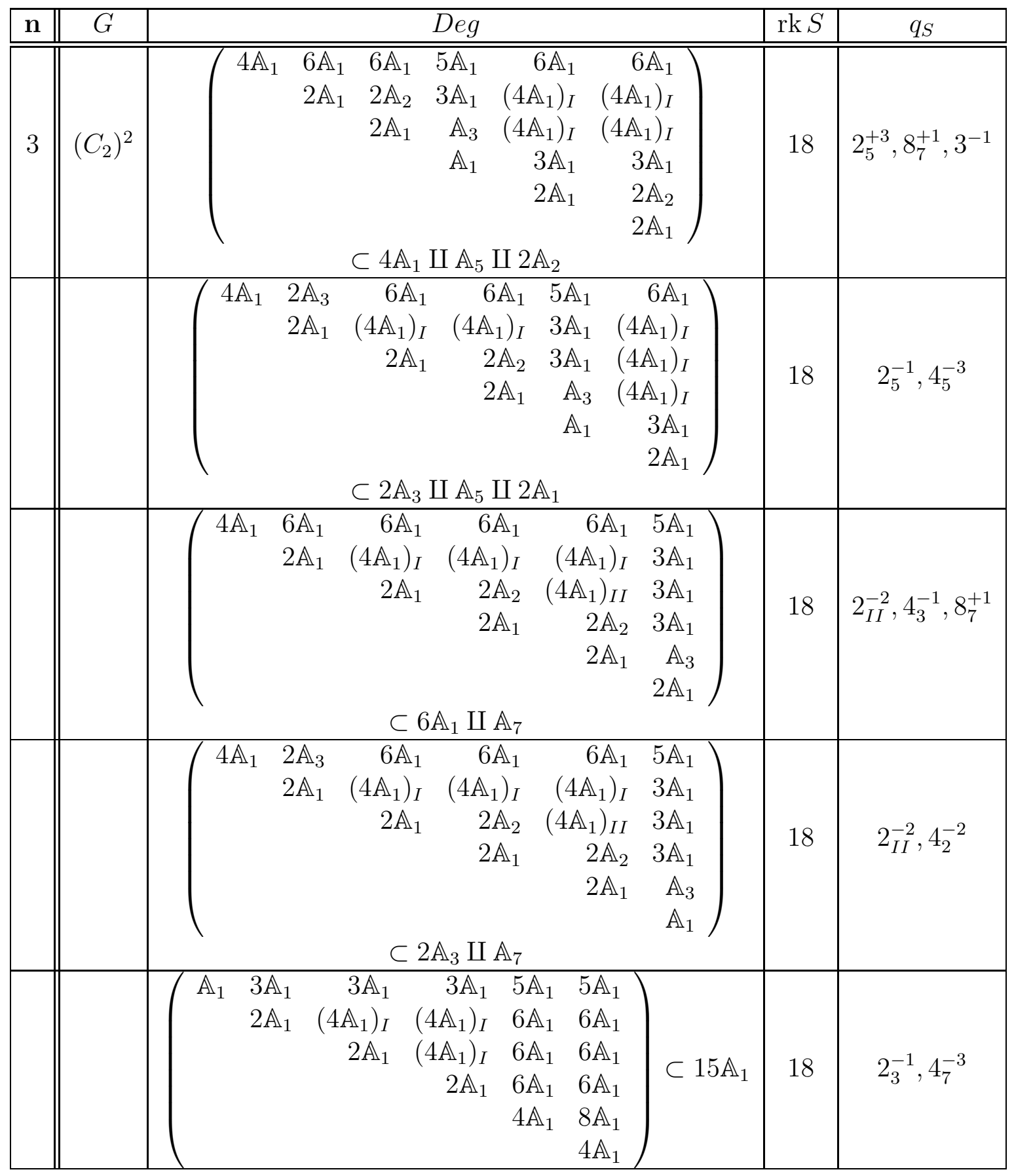




\begin{tabular}{|c|c|c|c|c|}
\hline n & $\bar{G}$ & $\overline{D e g}$ & $\operatorname{rk} S$ & $q_{S}$ \\
\hline \multirow[t]{6}{*}{3} & $\left(C_{2}\right)^{2}$ & $\left.\begin{array}{ccrrrr}\mathbb{A}_{1} & 3 \mathbb{A}_{1} & 3 \mathbb{A}_{1} & 3 \mathbb{A}_{1} & 5 \mathbb{A}_{1} & 5 \mathbb{A}_{1} \\
& 2 \mathbb{A}_{1} & \left(4 \mathbb{A}_{1}\right)_{I} & \left(4 \mathbb{A}_{1}\right)_{I} & 6 \mathbb{A}_{1} & 6 \mathbb{A}_{1} \\
& & 2 \mathbb{A}_{1} & \left(4 \mathbb{A}_{1}\right)_{I} & 6 \mathbb{A}_{1} & 6 \mathbb{A}_{1} \\
& & 2 \mathbb{A}_{1} & 6 \mathbb{A}_{1} & 6 \mathbb{A}_{1} \\
& & & & 4 \mathbb{A}_{1} & 4 \mathbb{A}_{2} \\
& & & & & 4 \mathbb{A}_{1}\end{array}\right)$ & 18 & $2_{1}^{+1}, 4_{7}^{+3}, 3^{+1}$ \\
\hline & & $\left(\mathbb{A}_{1}, 2 \mathbb{A}_{1}, 2 \mathbb{A}_{1}, 2 \mathbb{A}_{1}, 4 \mathbb{A}_{1}, 4 \mathbb{A}_{1}\right) \subset 3 \mathbb{A}_{1} \amalg 2 \mathbb{A}_{2} \amalg 4 \mathbb{A}_{2}$ & 18 & $2_{5}^{-3}, 4_{5}^{-1}, 3^{-2}$ \\
\hline & & 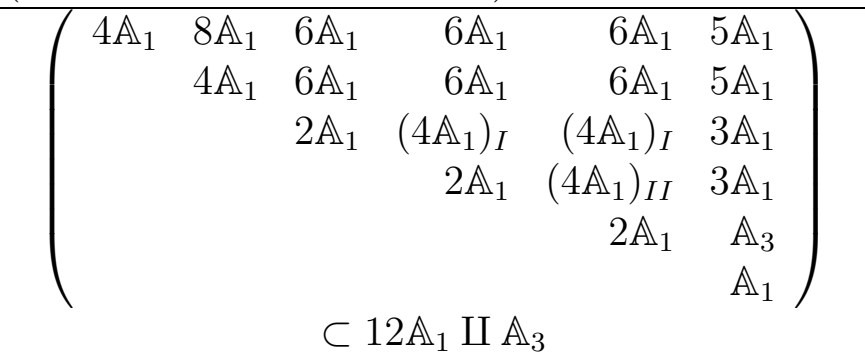 & 18 & $4_{6}^{+4}$ \\
\hline & & $\left(\begin{array}{rrrrrr}4 \mathbb{A}_{1} & 4 \mathbb{A}_{2} & 5 \mathbb{A}_{1} & 6 \mathbb{A}_{1} & 6 \mathbb{A}_{1} & 6 \mathbb{A}_{1} \\
& 4 \mathbb{A}_{1} & 5 \mathbb{A}_{1} & 6 \mathbb{A}_{1} & 6 \mathbb{A}_{1} & 6 \mathbb{A}_{1} \\
& & \mathbb{A}_{1} & \mathbb{A}_{3} & 3 \mathbb{A}_{1} & 3 \mathbb{A}_{1} \\
& & & 2 \mathbb{A}_{1} & \left(4 \mathbb{A}_{1}\right)_{I} & \left(4 \mathbb{A}_{1}\right)_{I} \\
& & & & 2 \mathbb{A}_{1} & \left(4 \mathbb{A}_{1}\right)_{I} \\
& & & & & 2 \mathbb{A}_{1}\end{array}\right)$ & 18 & $2_{0}^{+2}, 4_{0}^{+2}, 3^{+1}$ \\
\hline & & 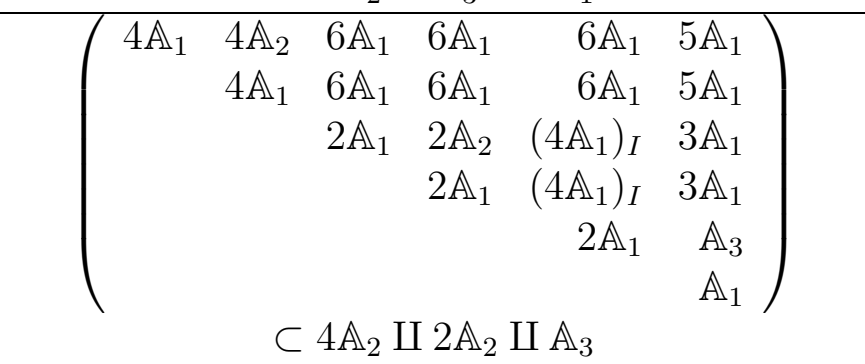 & 18 & $2_{2}^{-4}, 3^{-2}$ \\
\hline & & $\left.\begin{array}{cccrrr}4 \mathbb{A}_{1} & 2 \mathbb{A}_{3} & 8 \mathbb{A}_{1} & 6 \mathbb{A}_{1} & 6 \mathbb{A}_{1} & 5 \mathbb{A}_{1} \\
& 2 \mathbb{A}_{1} & 6 \mathbb{A}_{1} & \left(4 \mathbb{A}_{1}\right)_{I} & \left(4 \mathbb{A}_{1}\right)_{I} & 3 \mathbb{A}_{1} \\
& & 4 \mathbb{A}_{1} & 6 \mathbb{A}_{1} & 6 \mathbb{A}_{1} & 5 \mathbb{A}_{1} \\
& & & 2 \mathbb{A}_{1} & \left(4 \mathbb{A}_{1}\right)_{I} & 3 \mathbb{A}_{1} \\
& & & & 2 \mathbb{A}_{1} & 3 \mathbb{A}_{1} \\
& & & & & \mathbb{A}_{1}\end{array}\right)$ & 18 & $2_{3}^{-1}, 4_{6}^{-2}, 8_{5}^{-1}$ \\
\hline
\end{tabular}




\begin{tabular}{|c|c|c|c|c|}
\hline n & $G$ & $D e g$ & rk $S$ & $q_{S}$ \\
\hline \multirow[t]{5}{*}{3} & $\left(C_{2}\right)^{2}$ & $\left.\begin{array}{crrrrr}4 \mathbb{A}_{1} & 2 \mathbb{A}_{3} & 8 \mathbb{A}_{1} & 6 \mathbb{A}_{1} & 5 \mathbb{A}_{1} & 6 \mathbb{A}_{1} \\
& 2 \mathbb{A}_{1} & 6 \mathbb{A}_{1} & \left(4 \mathbb{A}_{1}\right)_{I} & 3 \mathbb{A}_{1} & \left(4 \mathbb{A}_{1}\right)_{I} \\
& & 4 \mathbb{A}_{1} & 6 \mathbb{A}_{1} & 5 \mathbb{A}_{1} & 6 \mathbb{A}_{1} \\
& & & 2 \mathbb{A}_{1} & \mathbb{A}_{3} & \left(4 \mathbb{A}_{1}\right)_{I} \\
& & & & \mathbb{A}_{1} & 3 \mathbb{A}_{1} \\
& & & & & 2 \mathbb{A}_{1}\end{array}\right)$ & 18 & $2_{6}^{-2}, 4_{5}^{-1}, 8_{3}^{-1}$ \\
\hline & & $\left(\begin{array}{crrrrr}4 \mathbb{A}_{1} & 2 \mathbb{A}_{3} & 8 \mathbb{A}_{1} & 6 \mathbb{A}_{1} & 5 \mathbb{A}_{1} & 6 \mathbb{A}_{1} \\
& 2 \mathbb{A}_{1} & 6 \mathbb{A}_{1} & \left(4 \mathbb{A}_{1}\right)_{I} & 3 \mathbb{A}_{1} & \left(4 \mathbb{A}_{1}\right)_{I} \\
& & 4 \mathbb{A}_{1} & 6 \mathbb{A}_{1} & 5 \mathbb{A}_{1} & 6 \mathbb{A}_{1} \\
& & & 2 \mathbb{A}_{1} & \mathbb{A}_{3} & \left(4 \mathbb{A}_{1}\right)_{I I} \\
& & & & \mathbb{A}_{1} & 3 \mathbb{A}_{1} \\
& & & & & 2 \mathbb{A}_{1}\end{array}\right)$ & 18 & $2_{I I}^{+2}, 4_{5}^{-1}, 8_{5}^{-1}$ \\
\hline & & $\left(\begin{array}{rrrrrr}4 \mathbb{A}_{1} & 2 \mathbb{A}_{3} & 8 \mathbb{A}_{1} & 6 \mathbb{A}_{1} & 6 \mathbb{A}_{1} & 5 \mathbb{A}_{1} \\
& 2 \mathbb{A}_{1} & 6 \mathbb{A}_{1} & \left(4 \mathbb{A}_{1}\right)_{I} & \left(4 \mathbb{A}_{1}\right)_{I} & 3 \mathbb{A}_{1} \\
& & 4 \mathbb{A}_{1} & 2 \mathbb{A}_{3} & 6 \mathbb{A}_{1} & 5 \mathbb{A}_{1} \\
& & & 2 \mathbb{A}_{1} & \left(4 \mathbb{A}_{1}\right)_{I} & 3 \mathbb{A}_{1} \\
& & & & 2 \mathbb{A}_{1} & 3 \mathbb{A}_{1} \\
& & & & & \mathbb{A}_{1}\end{array}\right)$ & 18 & $2_{5}^{-1}, 4_{5}^{-3}$ \\
\hline & & $\left(\begin{array}{rrrrrr}4 \mathbb{A}_{1} & 2 \mathbb{A}_{3} & 8 \mathbb{A}_{1} & 6 \mathbb{A}_{1} & 6 \mathbb{A}_{1} & 5 \mathbb{A}_{1} \\
& 2 \mathbb{A}_{1} & 6 \mathbb{A}_{1} & \left(4 \mathbb{A}_{1}\right)_{I} & \left(4 \mathbb{A}_{1}\right)_{I} & 3 \mathbb{A}_{1} \\
& & 4 \mathbb{A}_{1} & 2 \mathbb{A}_{3} & 6 \mathbb{A}_{1} & 5 \mathbb{A}_{1} \\
& & & 2 \mathbb{A}_{1} & \left(4 \mathbb{A}_{1}\right)_{I} & 3 \mathbb{A}_{1} \\
& & & & 2 \mathbb{A}_{1} & \mathbb{A}_{3} \\
& & & & & \mathbb{A}_{1}\end{array}\right)$ & 18 & $2_{2}^{-2}, 4_{I I}^{+2}$ \\
\hline & & $\left.\begin{array}{cccccc}4 \mathbb{A}_{1} & 2 \mathbb{A}_{3} & 6 \mathbb{A}_{1} & 8 \mathbb{A}_{1} & 6 \mathbb{A}_{1} & 5 \mathbb{A}_{1} \\
& 2 \mathbb{A}_{1} & 2 \mathbb{A}_{2} & 6 \mathbb{A}_{1} & \left(4 \mathbb{A}_{1}\right)_{I} & 3 \mathbb{A}_{1} \\
& & 2 \mathbb{A}_{1} & 6 \mathbb{A}_{1} & \left(4 \mathbb{A}_{1}\right)_{I} & 3 \mathbb{A}_{1} \\
& & & 4 \mathbb{A}_{1} & 6 \mathbb{A}_{1} & 5 \mathbb{A}_{1} \\
& & & & 2 \mathbb{A}_{1} & 3 \mathbb{A}_{1} \\
& & & & & \mathbb{A}_{1}\end{array}\right)$ & 18 & $2_{7}^{+1}, 4_{7}^{+3}$ \\
\hline
\end{tabular}




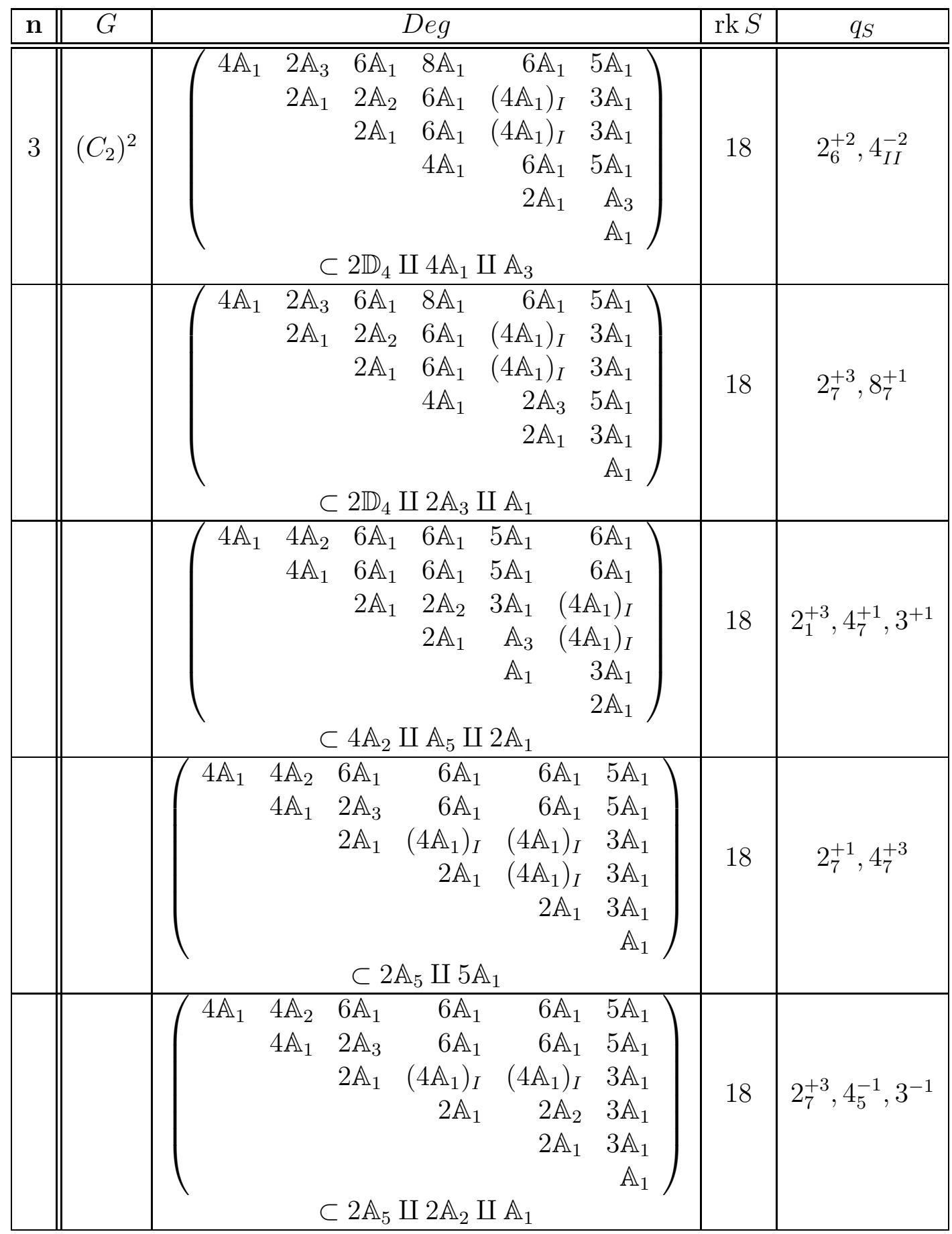




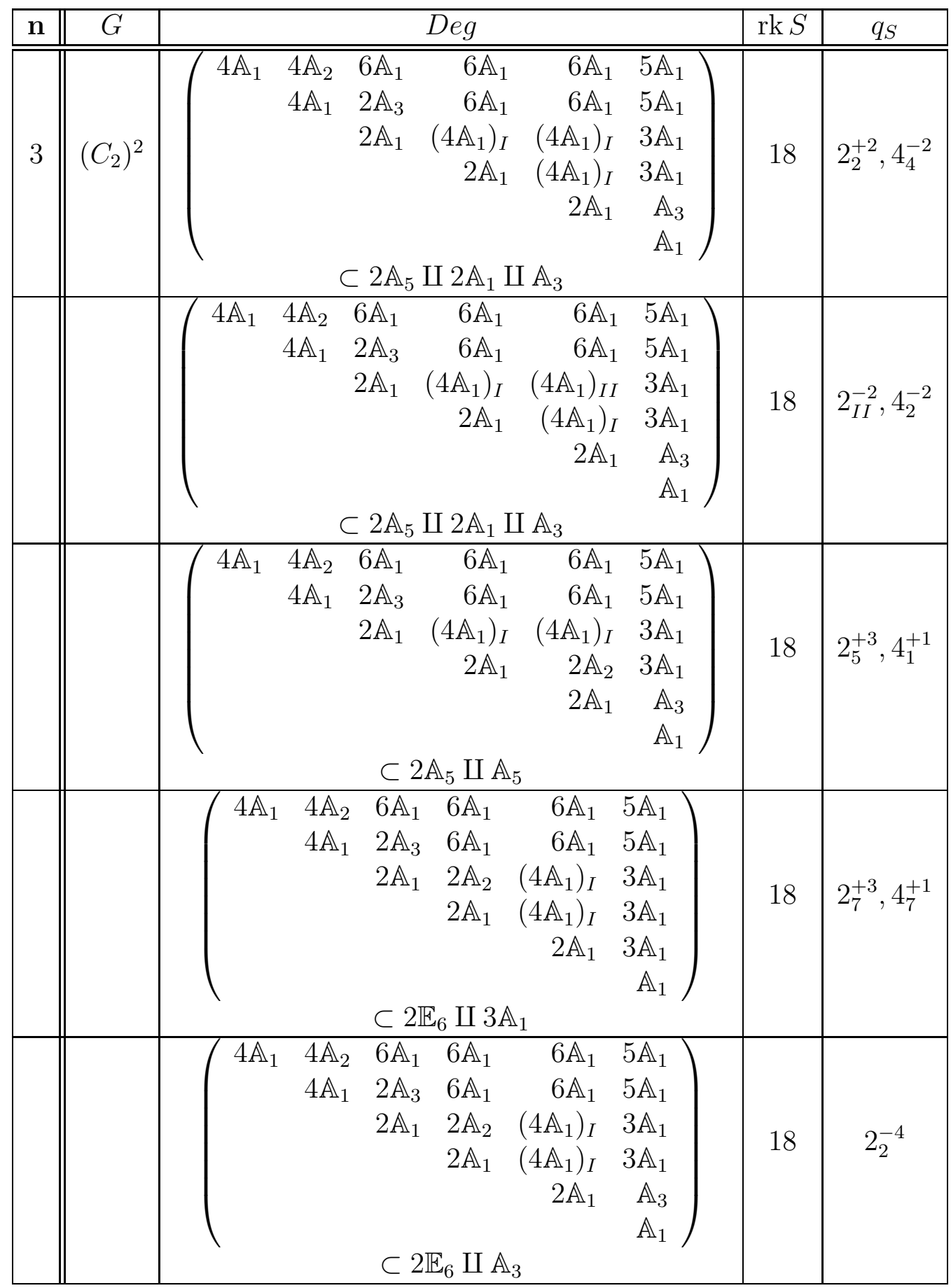




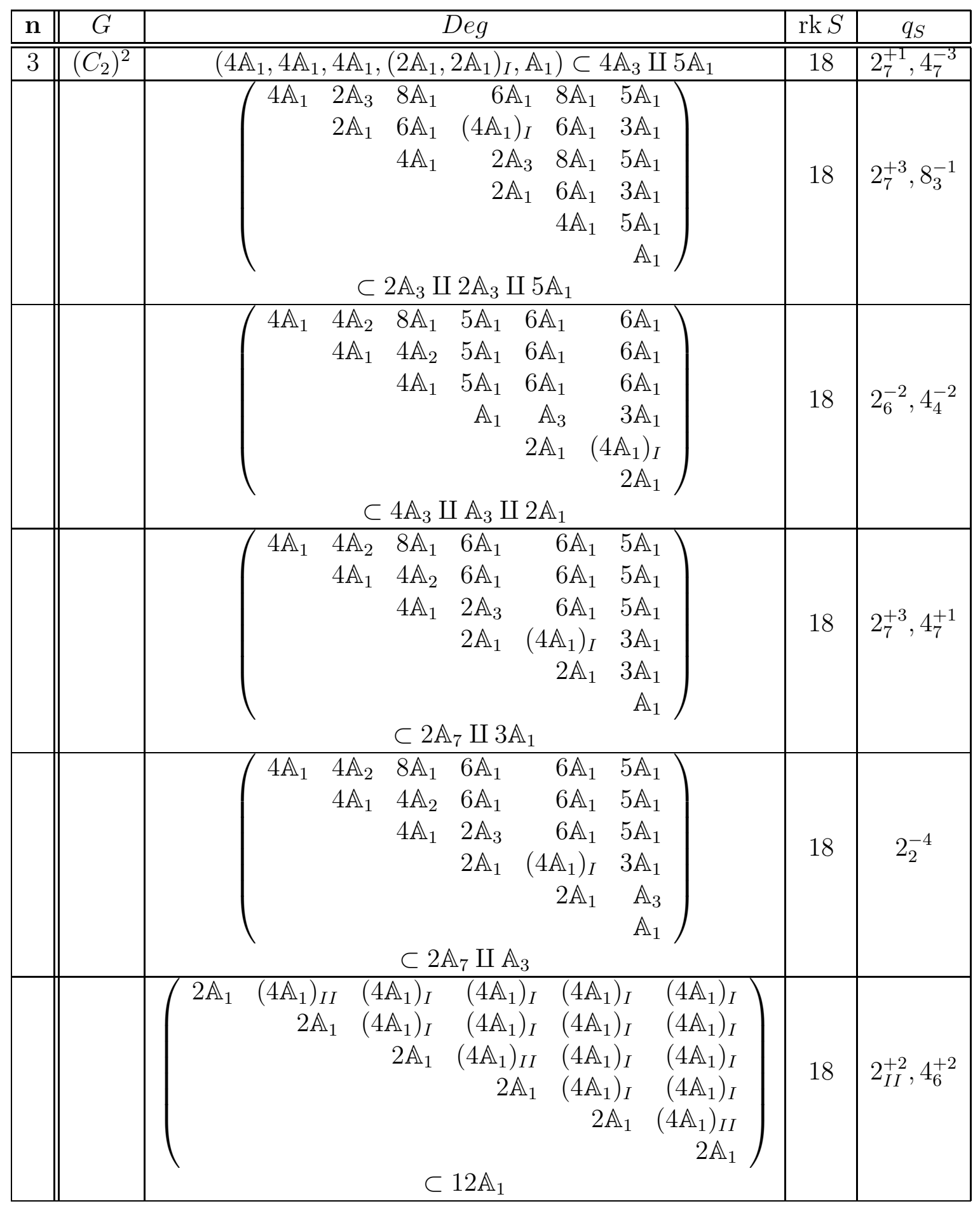




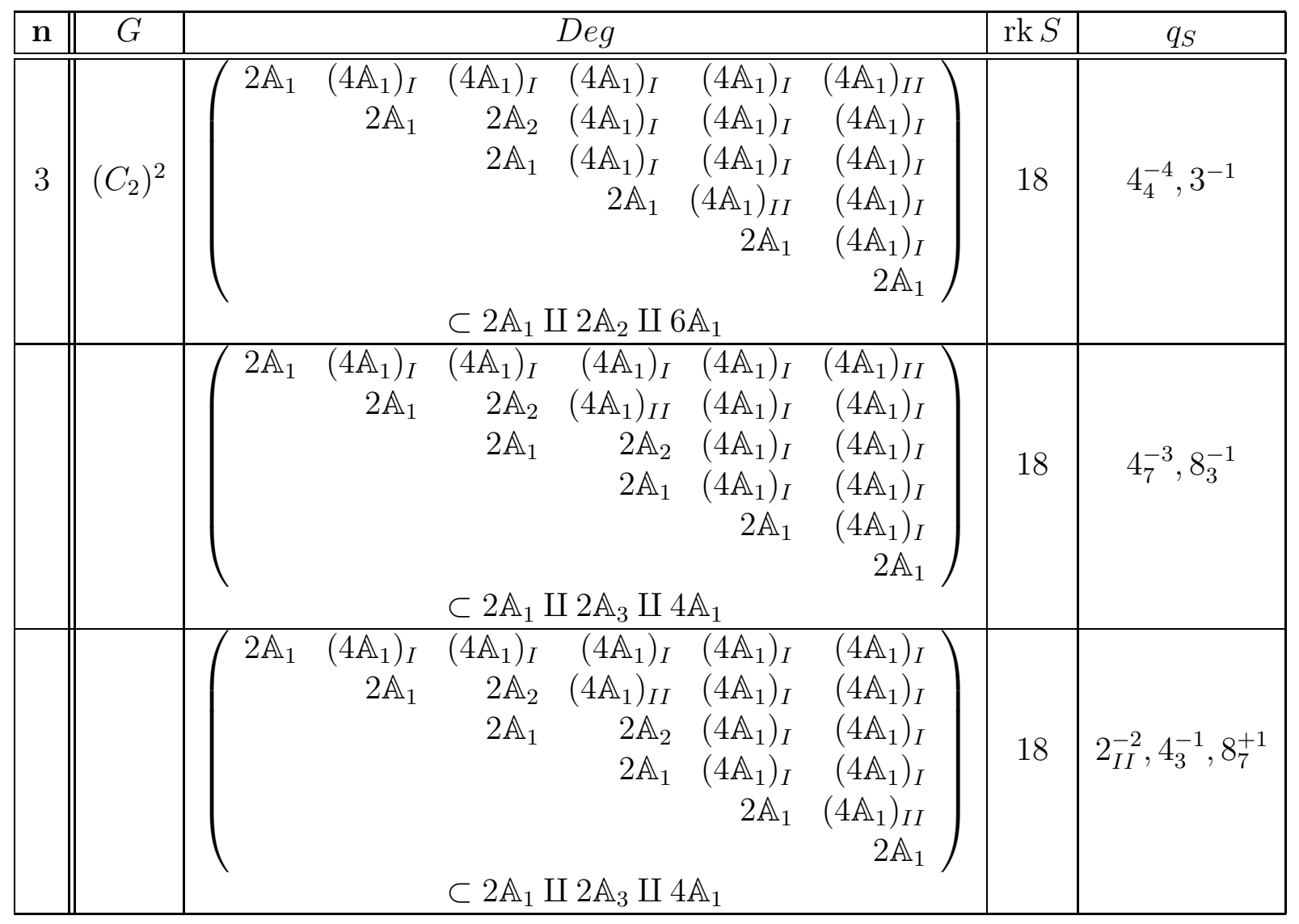




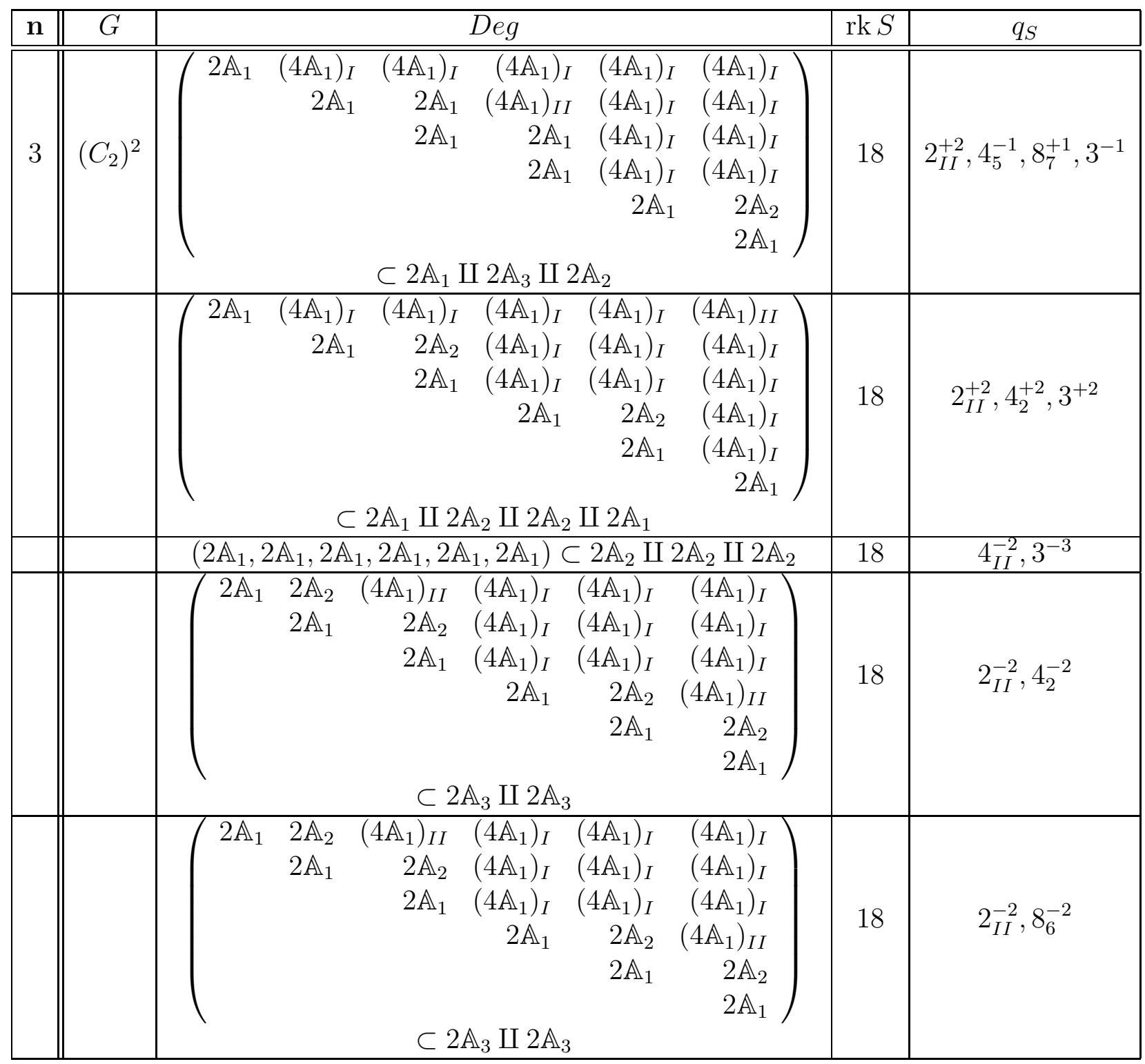




\begin{tabular}{|c|c|c|c|c|}
\hline $\mathbf{n}$ & $G$ & $\overline{D e g}$ & $\operatorname{rk} S$ & $q_{S}$ \\
\hline \multirow[t]{6}{*}{3} & $\left(C_{2}\right)^{2}$ & 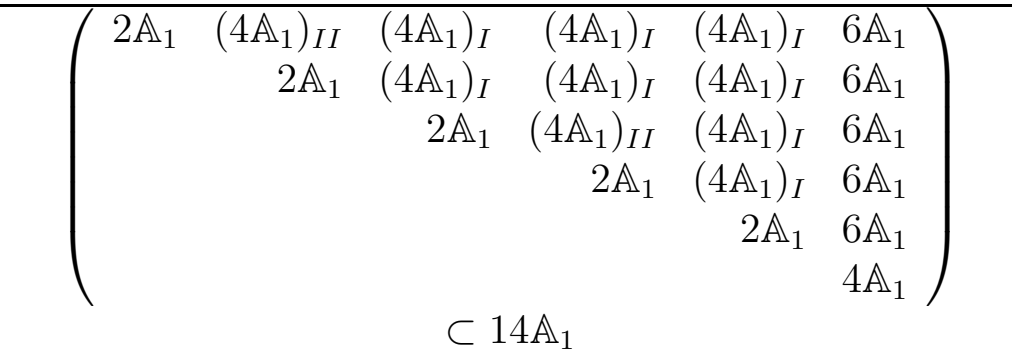 & 18 & $2_{I I}^{+2}, 4_{7}^{+1}, 8_{7}^{+1}$ \\
\hline & & $\left.\begin{array}{ccrrrr}4 \mathbb{A}_{1} & 6 \mathbb{A}_{1} & 6 \mathbb{A}_{1} & 6 \mathbb{A}_{1} & 6 \mathbb{A}_{1} & 6 \mathbb{A}_{1} \\
& 2 \mathbb{A}_{1} & 2 \mathbb{A}_{2} & \left(4 \mathbb{A}_{1}\right)_{I} & \left(4 \mathbb{A}_{1}\right)_{I} & \left(4 \mathbb{A}_{1}\right)_{I} \\
& & 2 \mathbb{A}_{1} & \left(4 \mathbb{A}_{1}\right)_{I} & \left(4 \mathbb{A}_{1}\right)_{I} & \left(4 \mathbb{A}_{1}\right)_{I} \\
& & & 2 \mathbb{A}_{1} & \left(4 \mathbb{A}_{1}\right)_{I I} & \left(4 \mathbb{A}_{1}\right)_{I} \\
& & & & 2 \mathbb{A}_{1} & \left(4 \mathbb{A}_{1}\right)_{I} \\
& & & & & 2 \mathbb{A}_{1}\end{array}\right)$ & 18 & $2_{I I}^{+2}, 4_{3}^{-1}, 8_{1}^{+1}, 3^{-1}$ \\
\hline & & $\left(2 \mathbb{A}_{1}, 2 \mathbb{A}_{1}, 2 \mathbb{A}_{1}, 2 \mathbb{A}_{1}, 2 \mathbb{A}_{1}, 2 \mathbb{A}_{1}\right) \subset 2 \mathbb{A}_{1} \amalg 2 \mathbb{A}_{2} \amalg 2 \mathbb{A}_{2} \amalg 4 \mathbb{A}_{1}$ & 18 & $4_{3}^{-1}, 8_{3}^{-1}, 3^{+2}$ \\
\hline & & $\left.\begin{array}{crrrrr}4 \mathbb{A}_{1} & 2 \mathbb{A}_{3} & 6 \mathbb{A}_{1} & 6 \mathbb{A}_{1} & 6 \mathbb{A}_{1} & 6 \mathbb{A}_{1} \\
& 2 \mathbb{A}_{1} & \left(4 \mathbb{A}_{1}\right)_{I I} & \left(4 \mathbb{A}_{1}\right)_{I} & \left(4 \mathbb{A}_{1}\right)_{I} & \left(4 \mathbb{A}_{1}\right)_{I} \\
& & 2 \mathbb{A}_{1} & \left(4 \mathbb{A}_{1}\right)_{I} & \left(4 \mathbb{A}_{1}\right)_{I} & \left(4 \mathbb{A}_{1}\right)_{I} \\
& & & 2 \mathbb{A}_{1} & \left(4 \mathbb{A}_{1}\right)_{I} & \left(4 \mathbb{A}_{1}\right)_{I} \\
& & & & 2 \mathbb{A}_{1} & \left(4 \mathbb{A}_{1}\right)_{I I} \\
& & & & & 2 \mathbb{A}_{1}\end{array}\right)$ & 18 & $4_{6}^{+4}$ \\
\hline & & $\left.\begin{array}{crrrrr}4 \mathbb{A}_{1} & 2 \mathbb{A}_{3} & 6 \mathbb{A}_{1} & 6 \mathbb{A}_{1} & 6 \mathbb{A}_{1} & 6 \mathbb{A}_{1} \\
& 2 \mathbb{A}_{1} & \left(4 \mathbb{A}_{1}\right)_{I} & \left(4 \mathbb{A}_{1}\right)_{I} & \left(4 \mathbb{A}_{1}\right)_{I} & \left(4 \mathbb{A}_{1}\right)_{I} \\
& & 2 \mathbb{A}_{1} & \left(4 \mathbb{A}_{1}\right)_{I I} & \left(4 \mathbb{A}_{1}\right)_{I} & \left(4 \mathbb{A}_{1}\right)_{I} \\
& & & 2 \mathbb{A}_{1} & \left(4 \mathbb{A}_{1}\right)_{I} & \left(4 \mathbb{A}_{1}\right)_{I} \\
& & & & 2 \mathbb{A}_{1} & \left(4 \mathbb{A}_{1}\right)_{I I} \\
& & & & & 2 \mathbb{A}_{1}\end{array}\right)$ & 18 & $2_{I I}^{+2}, 4_{6}^{+2}$ \\
\hline & & $\left.\begin{array}{crrrrr}4 \mathbb{A}_{1} & 2 \mathbb{A}_{3} & 6 \mathbb{A}_{1} & 6 \mathbb{A}_{1} & 6 \mathbb{A}_{1} & 6 \mathbb{A}_{1} \\
& 2 \mathbb{A}_{1} & \left(4 \mathbb{A}_{1}\right)_{I} & \left(4 \mathbb{A}_{1}\right)_{I} & \left(4 \mathbb{A}_{1}\right)_{I} & \left(4 \mathbb{A}_{1}\right)_{I I} \\
& & 2 \mathbb{A}_{1} & 2 \mathbb{A}_{2} & \left(4 \mathbb{A}_{1}\right)_{I} & \left(4 \mathbb{A}_{1}\right)_{I} \\
& & & 2 \mathbb{A}_{1} & \left(4 \mathbb{A}_{1}\right)_{I} & \left(4 \mathbb{A}_{1}\right)_{I} \\
& & & & 2 \mathbb{A}_{1} & \left(4 \mathbb{A}_{1}\right)_{I} \\
& & & & & 2 \mathbb{A}_{1}\end{array}\right)$ & 18 & $2_{I I}^{+2}, 4_{4}^{-2}, 3^{-1}$ \\
\hline
\end{tabular}




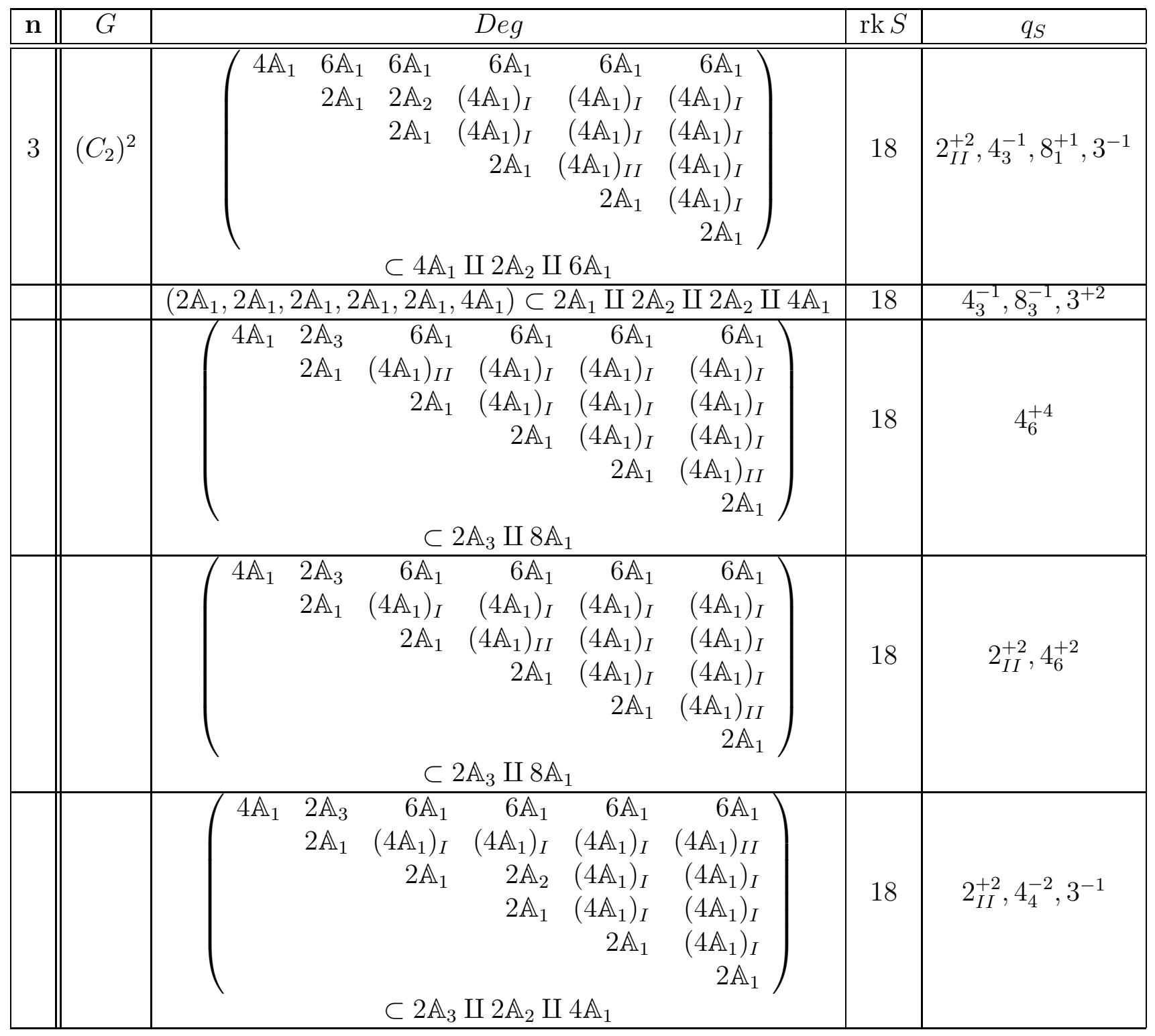




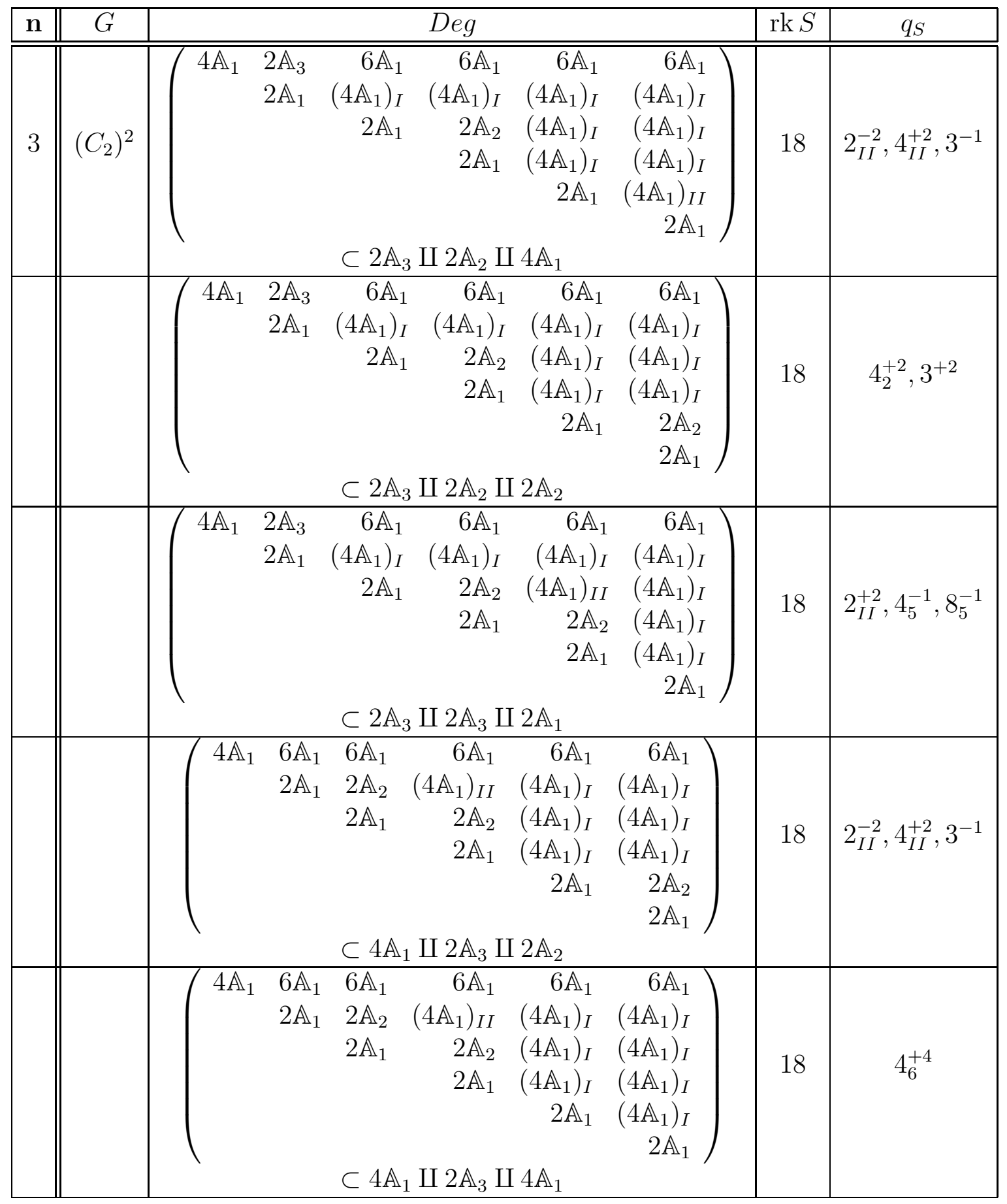




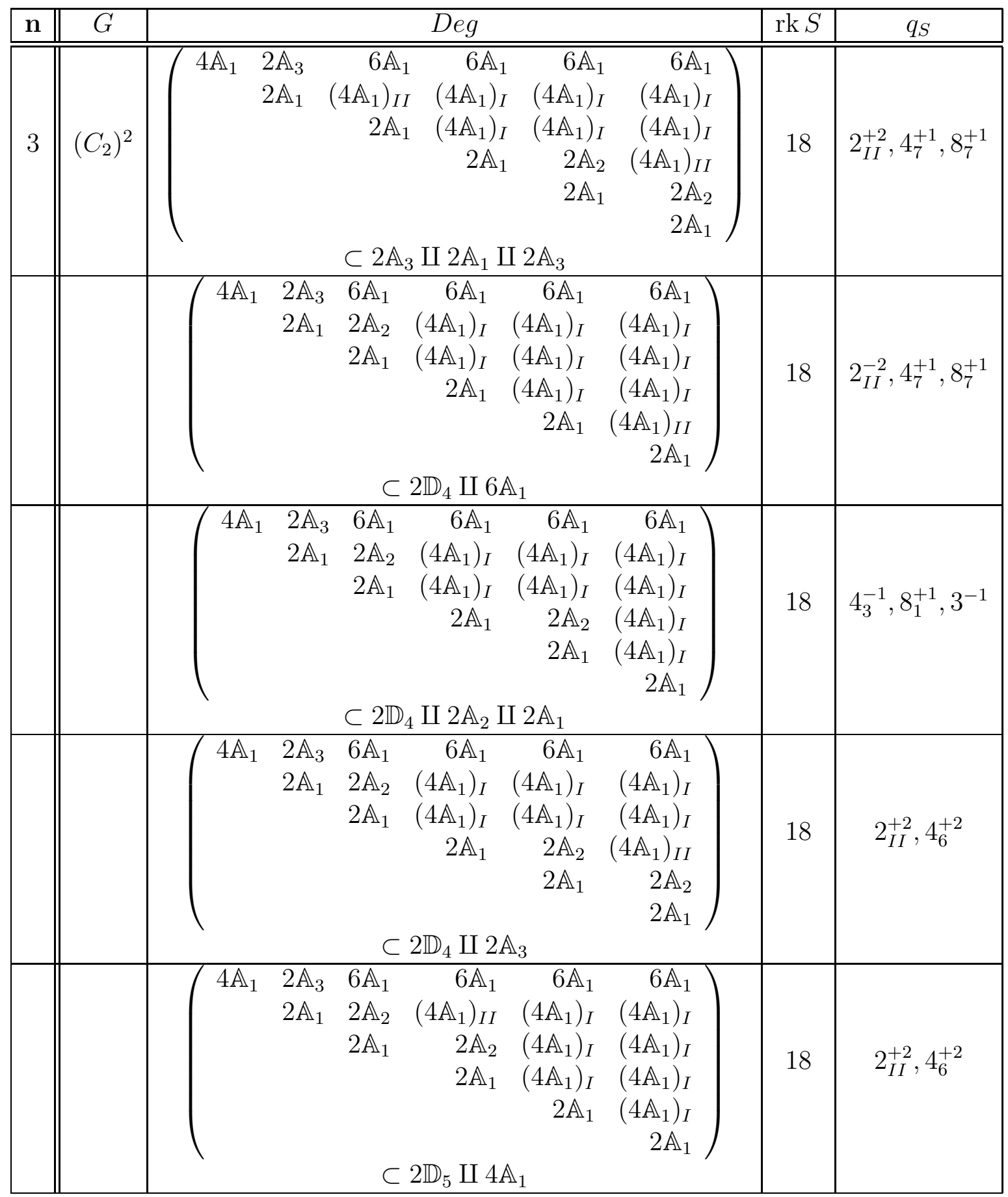




\begin{tabular}{|c|c|c|c|c|}
\hline n & $\bar{G}$ & $\overline{D e g}$ & $\operatorname{rk} S$ & $q_{S}$ \\
\hline \multirow[t]{5}{*}{3} & $\left(C_{2}\right)^{2}$ & 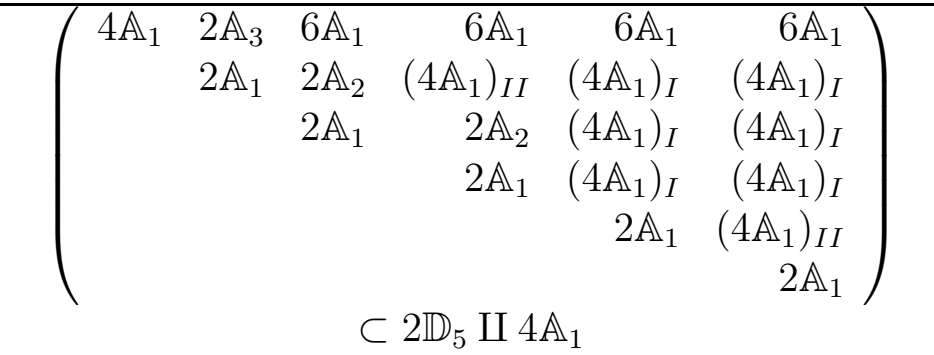 & 18 & $2_{I I}^{+2}, 4_{6}^{-2}$ \\
\hline & & 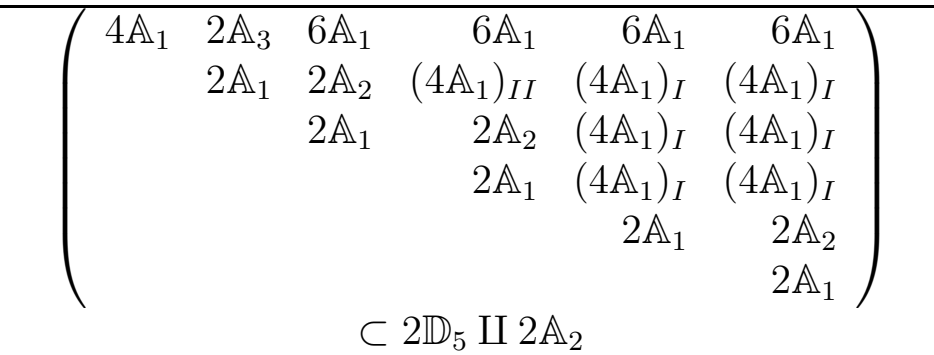 & 18 & $2_{I I}^{-4}, 3^{-1}$ \\
\hline & & $\left.\begin{array}{cccrrr}4 \mathbb{A}_{1} & 6 \mathbb{A}_{1} & 6 \mathbb{A}_{1} & 6 \mathbb{A}_{1} & 6 \mathbb{A}_{1} & 6 \mathbb{A}_{1} \\
& 2 \mathbb{A}_{1} & 2 \mathbb{A}_{2} & \left(4 \mathbb{A}_{1}\right)_{I I} & \left(4 \mathbb{A}_{1}\right)_{I} & \left(4 \mathbb{A}_{1}\right)_{I} \\
& & 2 \mathbb{A}_{1} & 2 \mathbb{A}_{2} & \left(4 \mathbb{A}_{1}\right)_{I} & \left(4 \mathbb{A}_{1}\right)_{I} \\
& & & 2 \mathbb{A}_{1} & \left(4 \mathbb{A}_{1}\right)_{I} & \left(4 \mathbb{A}_{1}\right)_{I} \\
& & & & 2 \mathbb{A}_{1} & \left(4 \mathbb{A}_{1}\right)_{I I} \\
& & & & & 2 \mathbb{A}_{1}\end{array}\right)$ & 18 & $2_{I I}^{-2}, 4_{2}^{-2}$ \\
\hline & & $\left(\begin{array}{rrrrrr}2 \mathbb{A}_{1} & \left(4 \mathbb{A}_{1}\right)_{I I} & \left(4 \mathbb{A}_{1}\right)_{I} & \left(4 \mathbb{A}_{1}\right)_{I} & 6 \mathbb{A}_{1} & 6 \mathbb{A}_{1} \\
& 2 \mathbb{A}_{1} & \left(4 \mathbb{A}_{1}\right)_{I} & \left(4 \mathbb{A}_{1}\right)_{I} & 6 \mathbb{A}_{1} & 6 \mathbb{A}_{1} \\
& & 2 \mathbb{A}_{1} & \left(4 \mathbb{A}_{1}\right)_{I I} & 6 \mathbb{A}_{1} & 6 \mathbb{A}_{1} \\
& & 2 \mathbb{A}_{1} & 6 \mathbb{A}_{1} & 6 \mathbb{A}_{1} \\
& & & & 4 \mathbb{A}_{1} & 8 \mathbb{A}_{1} \\
& & & & & 4 \mathbb{A}_{1}\end{array}\right)$ & 18 & $2_{I I}^{+2}, 4_{6}^{+2}$ \\
\hline & & $\frac{\left(\left(2 \mathbb{A}_{1}, 2 \mathbb{A}_{1}\right)_{I}, 2 \mathbb{A}_{1}, 2 \mathbb{A}_{1}, 4 \mathbb{A}_{1}, 4 \mathbb{A}_{1}\right) \subset 4 \mathbb{A}_{1} \amalg 2 \mathbb{A}_{2} \amalg 4 \mathbb{A}_{2}}{\left(2 \mathbb{A}_{1}, 2 \mathbb{A}_{1}, 2 \mathbb{A}_{1}, 2 \mathbb{A}_{1}, 4 \mathbb{A}_{1}, 4 \mathbb{A}_{1}\right) \subset 2 \mathbb{A}_{2} \amalg 2 \mathbb{A}_{2} \amalg 4 \mathbb{A}_{2}}$ & $\frac{18}{18}$ & $\frac{4_{6}^{+2}, 3^{-2}}{2_{I I}^{-2}, 3^{+3}}$ \\
\hline
\end{tabular}




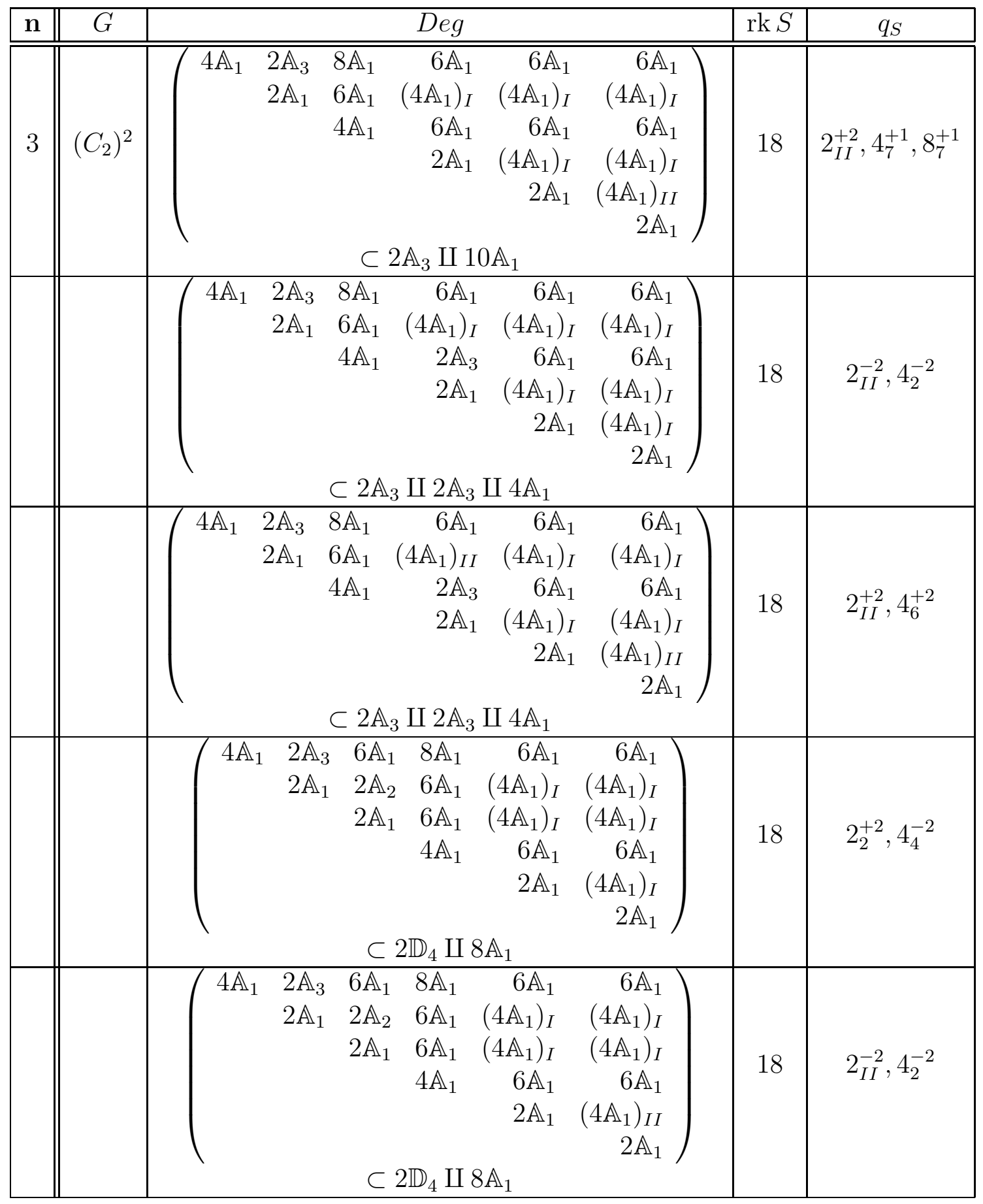




\section{Final remarks}

We hope to consider similar classification of degenerations and Picard lattices $S$ of Kählerian K3 surfaces with remaining small symplectic automorphism groups $\left(C_{2}\right)^{2}, C_{3}, C_{2}$ and $C_{1}$ later as well. Now, for these groups we obtain

Theorem 1. If the Picard lattice $S=M S_{X}$ of a K3 surface $X$ with finite symplectic automorphism group and with at least one -2 curve is different from all lattices of lines of Tables 1-4 of Section 4 from [18], lattices of Table 1 from [19] and lattices of Table 1 of this paper which are not denoted by o (for example, if its genus is different), then the symplectic automorphism group of $X$ is one of groups $\left(C_{2}\right)^{2}, C_{3}, C_{2}$ or $C_{1}$. 


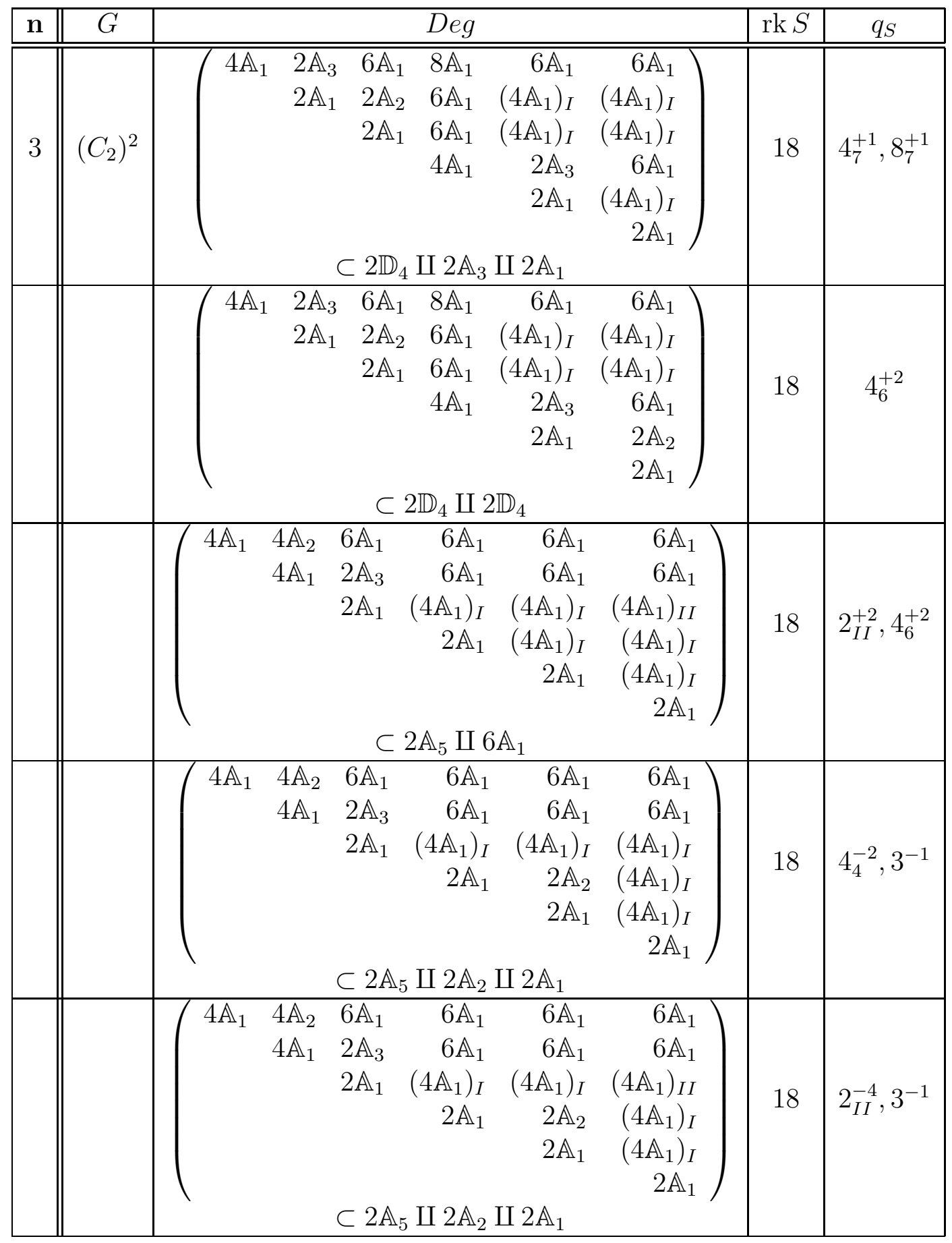




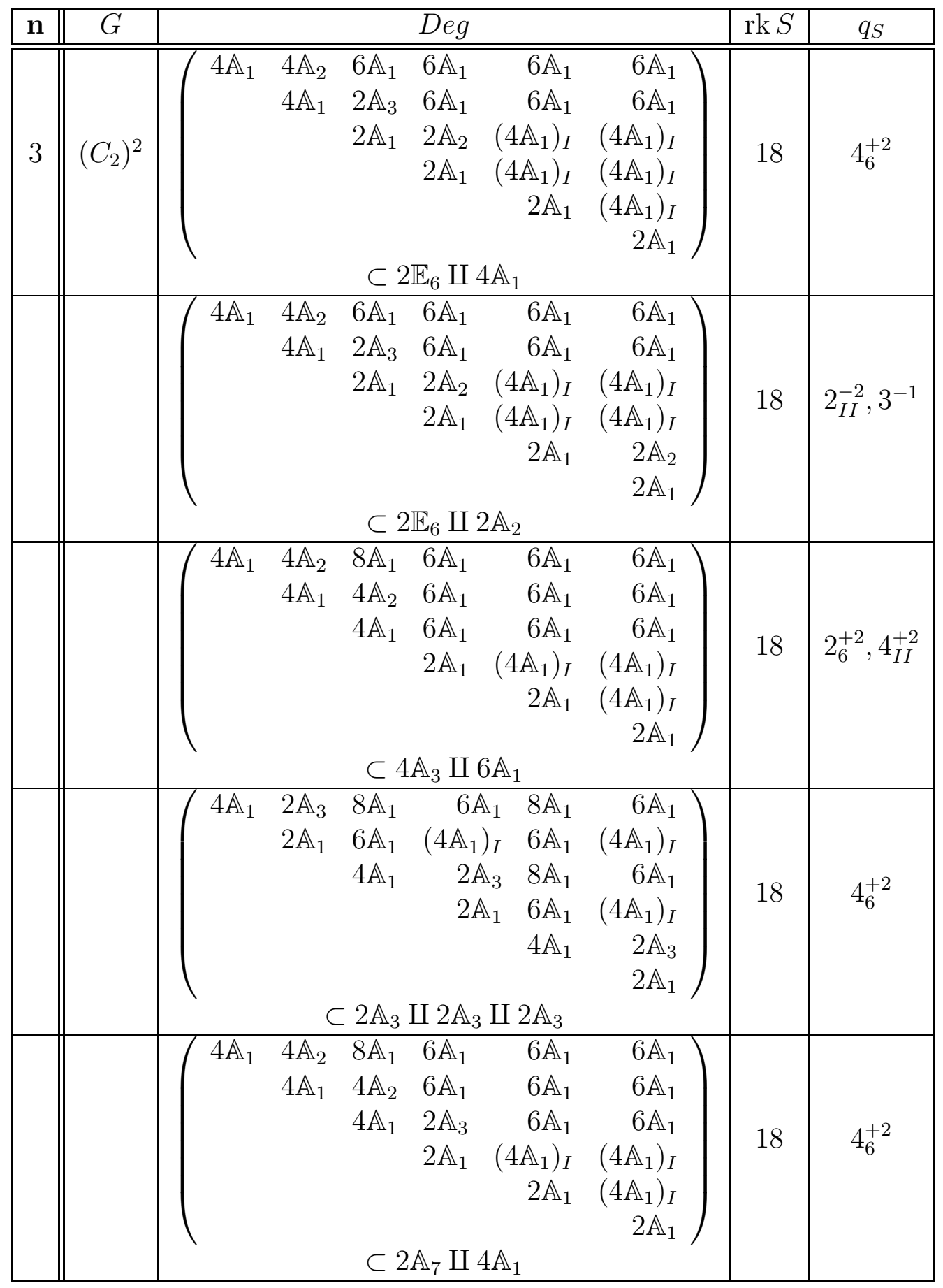




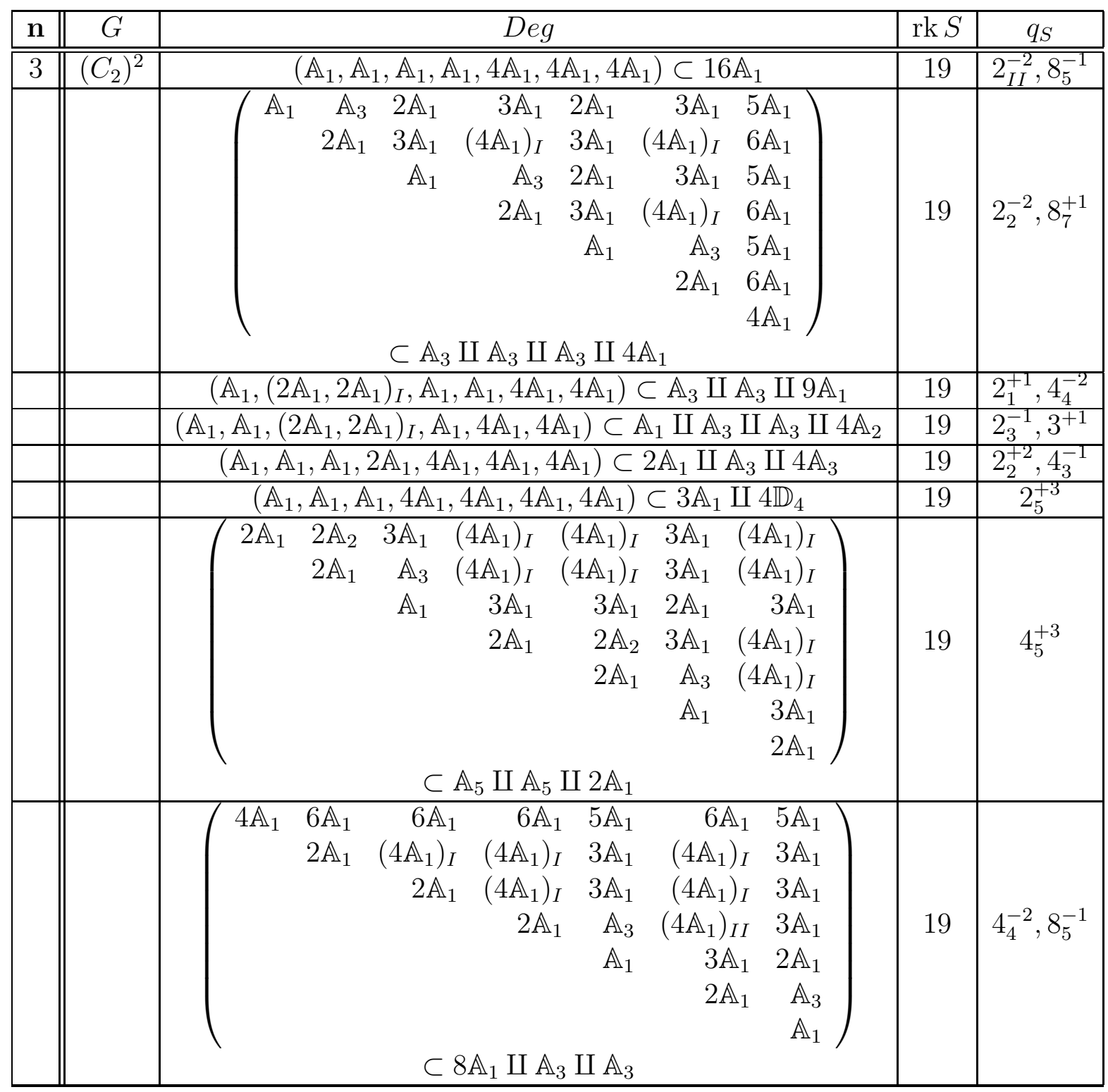




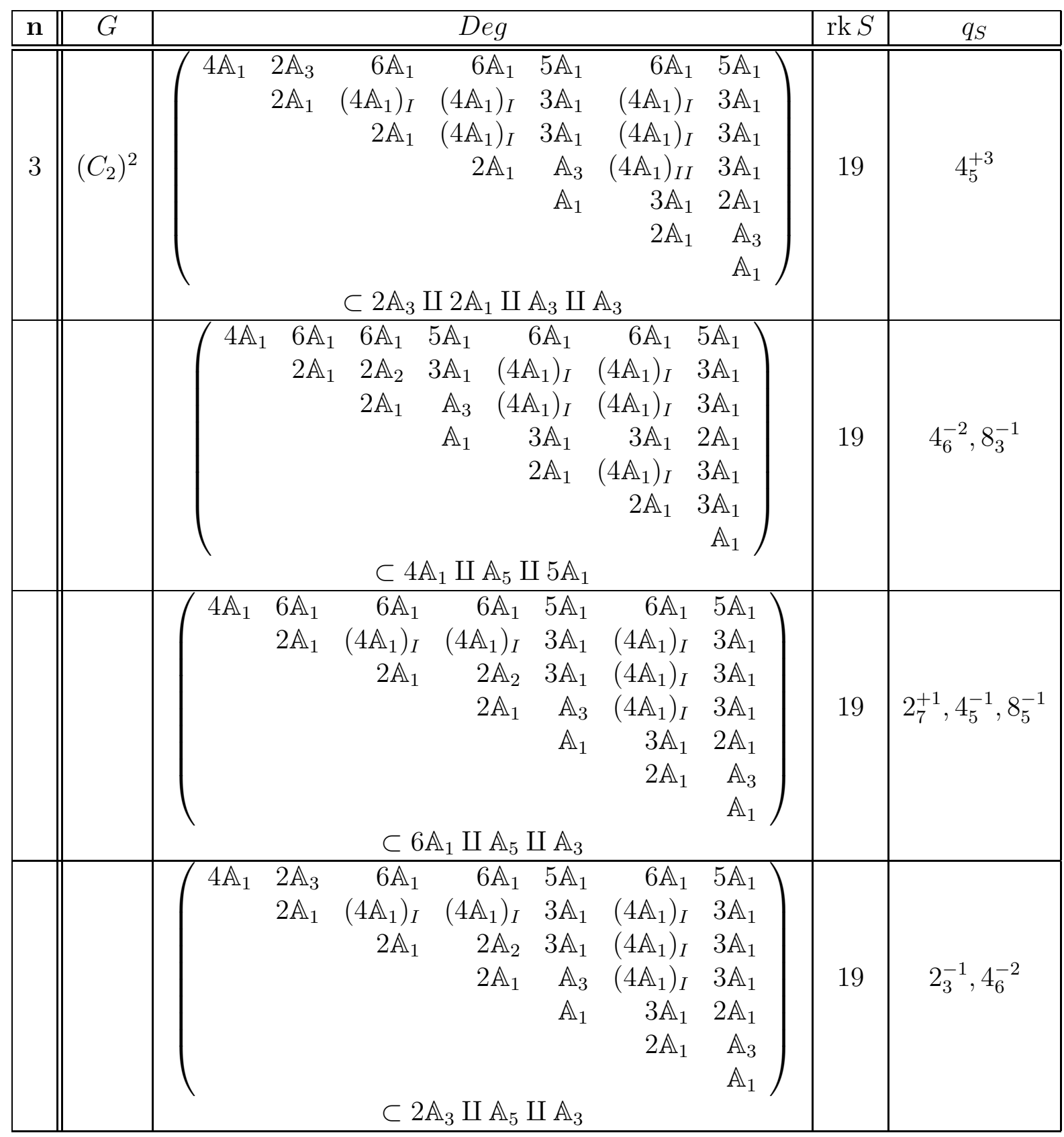




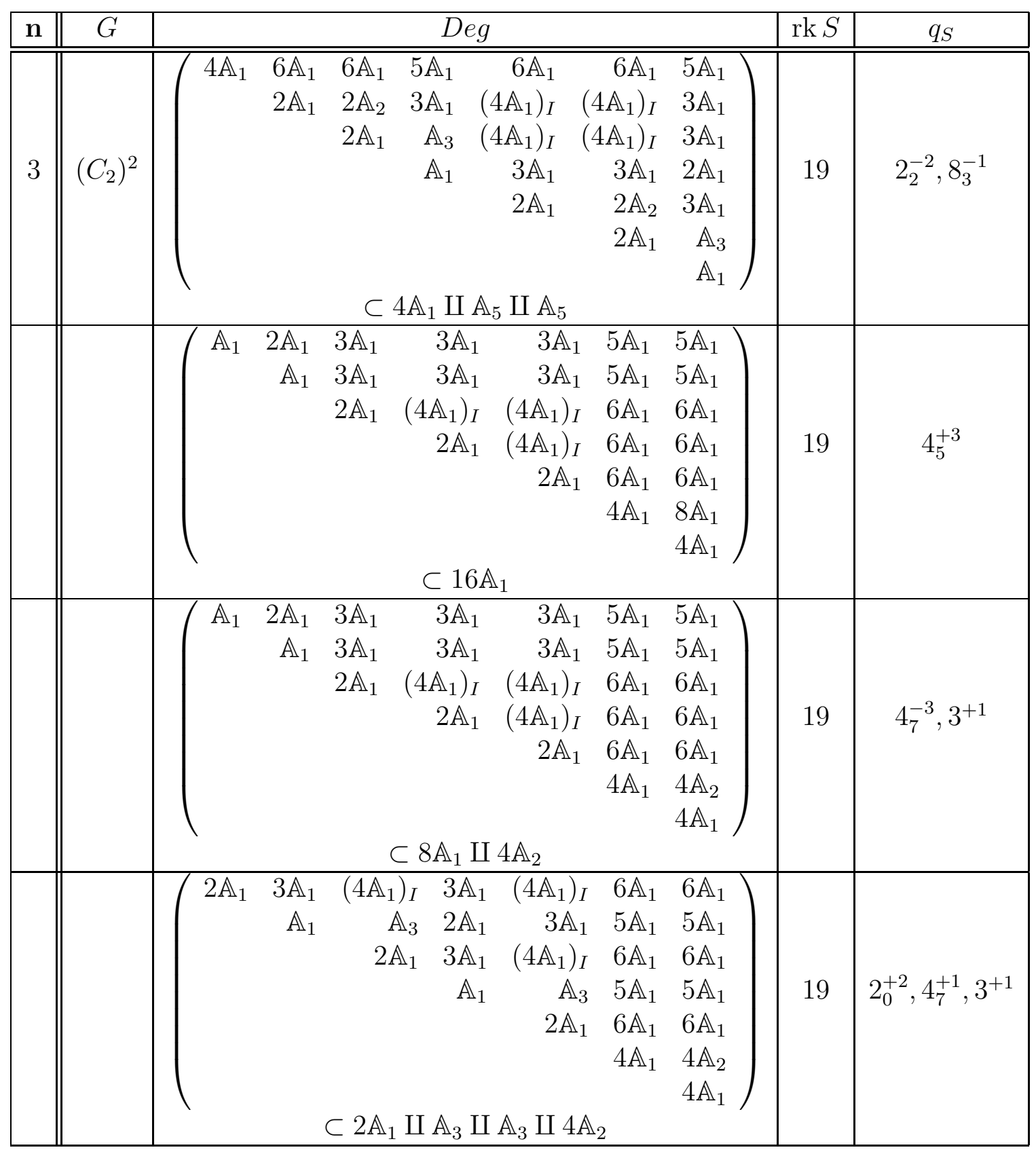




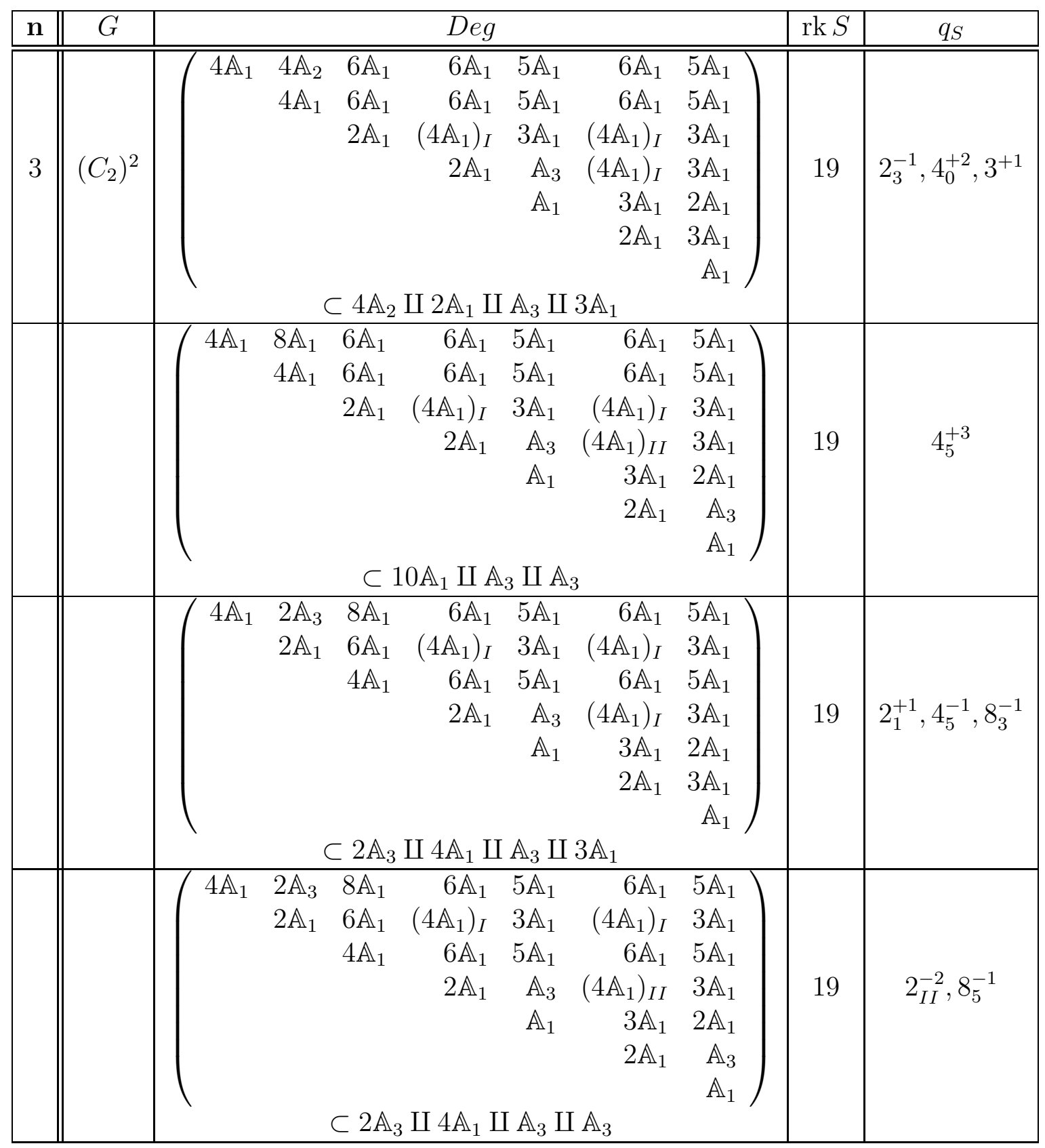




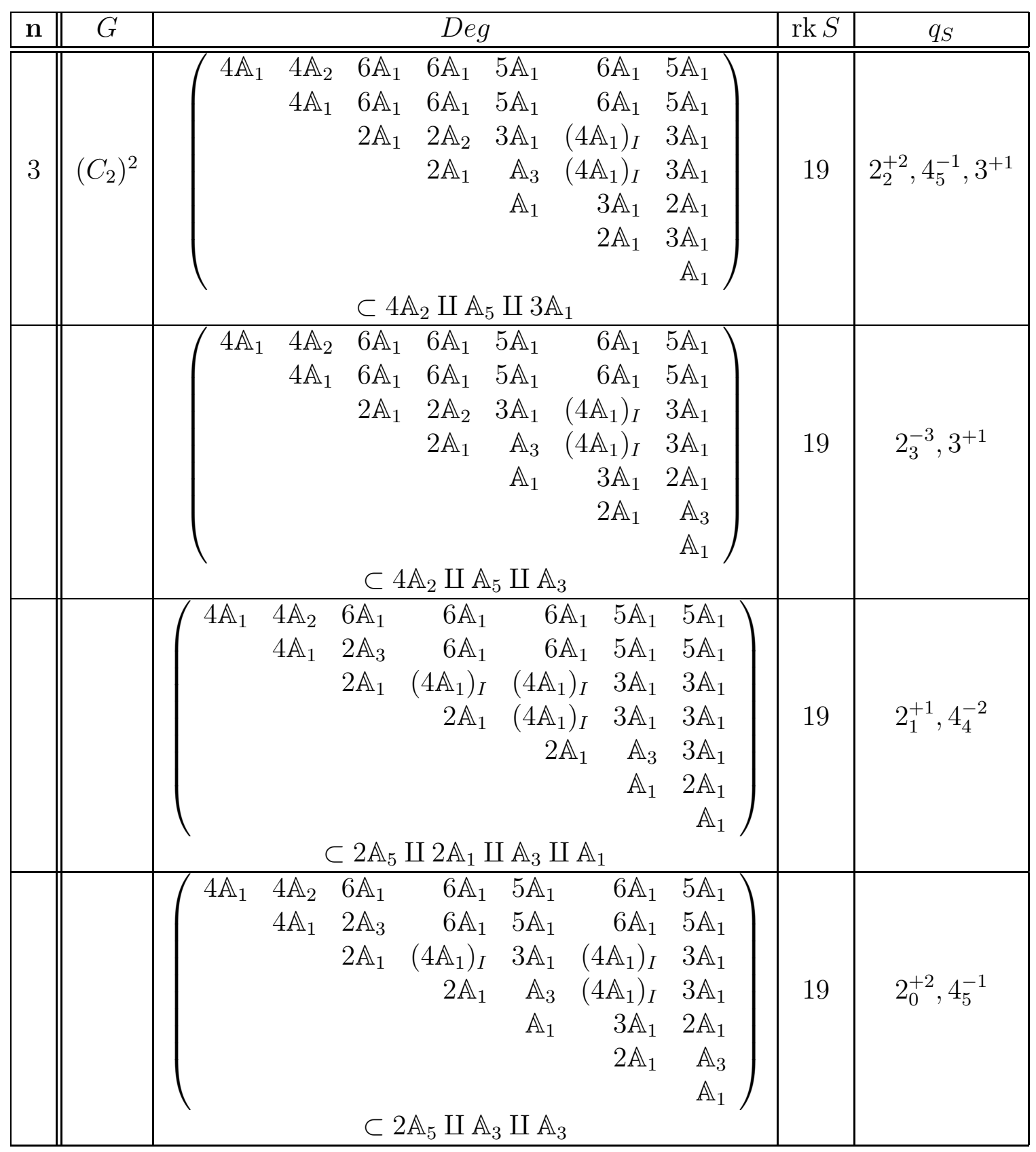




\begin{tabular}{|c|c|c|c|c|}
\hline n & $\bar{G}$ & $\overline{D e g}$ & $\operatorname{rk} S$ & $q_{S}$ \\
\hline \multirow[t]{8}{*}{3} & $\left(C_{2}\right)^{2}$ & $\left.\begin{array}{cccrrrr}4 \mathbb{A}_{1} & 4 \mathbb{A}_{2} & 6 \mathbb{A}_{1} & 6 \mathbb{A}_{1} & 6 \mathbb{A}_{1} & 5 \mathbb{A}_{1} & 5 \mathbb{A}_{1} \\
& 4 \mathbb{A}_{1} & 2 \mathbb{A}_{3} & 6 \mathbb{A}_{1} & 6 \mathbb{A}_{1} & 5 \mathbb{A}_{1} & 5 \mathbb{A}_{1} \\
& & 2 \mathbb{A}_{1} & \left(4 \mathbb{A}_{1}\right)_{I} & \left(4 \mathbb{A}_{1}\right)_{I} & 3 \mathbb{A}_{1} & 3 \mathbb{A}_{1} \\
& & & 2 \mathbb{A}_{1} & 2 \mathbb{A}_{2} & 3 \mathbb{A}_{1} & 3 \mathbb{A}_{1} \\
& & & & 2 \mathbb{A}_{1} & \mathbb{A}_{3} & 3 \mathbb{A}_{1} \\
& & & & & \mathbb{A}_{1} & 2 \mathbb{A}_{1} \\
& & & & & & \mathbb{A}_{1}\end{array}\right)$ & 19 & $2_{2}^{+2}, 4_{3}^{-1}$ \\
\hline & & $\left(4 \mathbb{A}_{1},\left(2 \mathbb{A}_{1}, 2 \mathbb{A}_{1}\right)_{I}, 4 \mathbb{A}_{1}, 4 \mathbb{A}_{1}, \mathbb{A}_{1}, \mathbb{A}_{1}\right) \subset 2 \mathbb{A}_{3} \amalg 2 \mathbb{A}_{3} \amalg 6 \mathbb{A}_{1}$ & 19 & $2_{2}^{-2}, 8_{3}^{-1}$ \\
\hline & & $\left(\mathbb{A}_{1}, \mathbb{A}_{1},\left(2 \mathbb{A}_{1}, 2 \mathbb{A}_{1}\right)_{I}, 4 \mathbb{A}_{1}, 4 \mathbb{A}_{1}, 4 \mathbb{A}_{1}\right) \subset 6 \mathbb{A}_{1} \amalg 4 \mathbb{A}_{3}$ & 19 & $4_{5}^{+3}$ \\
\hline & & $\left(\mathbb{A}_{1},\left(2 \mathbb{A}_{1}, 2 \mathbb{A}_{1}\right)_{I}, \mathbb{A}_{1}, 4 \mathbb{A}_{1}, 4 \mathbb{A}_{1}, 4 \mathbb{A}_{1}\right) \subset 3 \mathbb{A}_{1} \amalg \mathbb{A}_{3} \amalg 4 \mathbb{A}_{3}$ & 19 & $2_{5}^{-1}, 4_{4}^{-2}$ \\
\hline & & $\left(\mathbb{A}_{1},\left(2 \mathbb{A}_{1}, 2 \mathbb{A}_{1}\right)_{I}, \mathbb{A}_{1}, 4 \mathbb{A}_{1}, 4 \mathbb{A}_{1}, 4 \mathbb{A}_{1}\right) \subset \mathbb{A}_{3} \amalg \mathbb{A}_{3} \amalg 4 \mathbb{A}_{3}$ & 19 & $2_{6}^{-2}, 4_{3}^{-1}$ \\
\hline & & $\left.\begin{array}{rrrrrrr}4 \mathbb{A}_{1} & 4 \mathbb{A}_{2} & 6 \mathbb{A}_{1} & 6 \mathbb{A}_{1} & 6 \mathbb{A}_{1} & 5 \mathbb{A}_{1} & 5 \mathbb{A}_{1} \\
& 4 \mathbb{A}_{1} & 4 \mathbb{A}_{2} & 6 \mathbb{A}_{1} & 6 \mathbb{A}_{1} & 5 \mathbb{A}_{1} & 5 \mathbb{A}_{1} \\
& & 4 \mathbb{A}_{1} & 2 \mathbb{A}_{3} & 6 \mathbb{A}_{1} & 5 \mathbb{A}_{1} & 5 \mathbb{A}_{1} \\
& & & 2 \mathbb{A}_{1} & \left(4 \mathbb{A}_{1}\right)_{I} & 3 \mathbb{A}_{1} & 3 \mathbb{A}_{1} \\
& & & & 2 \mathbb{A}_{1} & 3 \mathbb{A}_{1} & 3 \mathbb{A}_{1} \\
& & & & & \mathbb{A}_{1} & 2 \mathbb{A}_{1} \\
& & & & & & \mathbb{A}_{1}\end{array}\right)$ & 19 & $2_{6}^{+2}, 4_{7}^{+1}$ \\
\hline & & $\left(\begin{array}{ccccrcc}4 \mathbb{A}_{1} & 4 \mathbb{A}_{2} & 8 \mathbb{A}_{1} & 6 \mathbb{A}_{1} & 6 \mathbb{A}_{1} & 5 \mathbb{A}_{1} & 5 \mathbb{A}_{1} \\
& 4 \mathbb{A}_{1} & 4 \mathbb{A}_{2} & 6 \mathbb{A}_{1} & 6 \mathbb{A}_{1} & 5 \mathbb{A}_{1} & 5 \mathbb{A}_{1} \\
& & 4 \mathbb{A}_{1} & 2 \mathbb{A}_{3} & 6 \mathbb{A}_{1} & 5 \mathbb{A}_{1} & 5 \mathbb{A}_{1} \\
& & & 2 \mathbb{A}_{1} & \left(4 \mathbb{A}_{1}\right)_{I} & 3 \mathbb{A}_{1} & 3 \mathbb{A}_{1} \\
& & & & 2 \mathbb{A}_{1} & \mathbb{A}_{3} & 3 \mathbb{A}_{1} \\
& & & & & \mathbb{A}_{1} & 2 \mathbb{A}_{1} \\
& & & & & & \mathbb{A}_{1}\end{array}\right)$ & 19 & $2_{5}^{+3}$ \\
\hline & & $\begin{array}{c}\left(\mathbb{A}_{1}, 2 \mathbb{A}_{1}, 2 \mathbb{A}_{1}, 2 \mathbb{A}_{1}, 2 \mathbb{A}_{1}, 2 \mathbb{A}_{1}, 2 \mathbb{A}_{1}\right) \\
\subset \mathbb{A}_{1} \amalg 2 \mathbb{A}_{2} \amalg 2 \mathbb{A}_{2} \amalg 2 \mathbb{A}_{2}\end{array}$ & 19 & $2_{3}^{-1}, 4_{I I}^{+2}, 3^{+1}$ \\
\hline
\end{tabular}




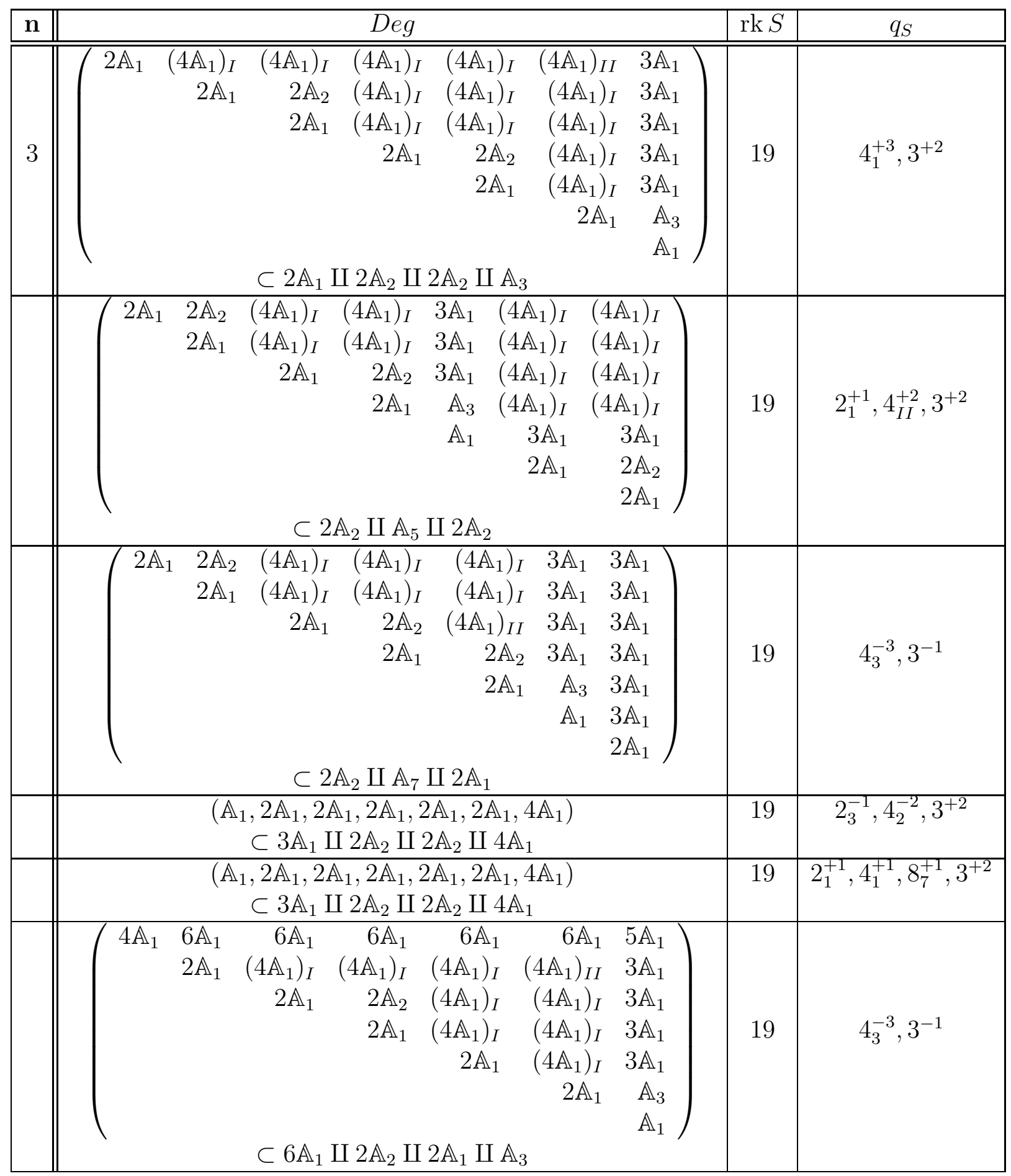




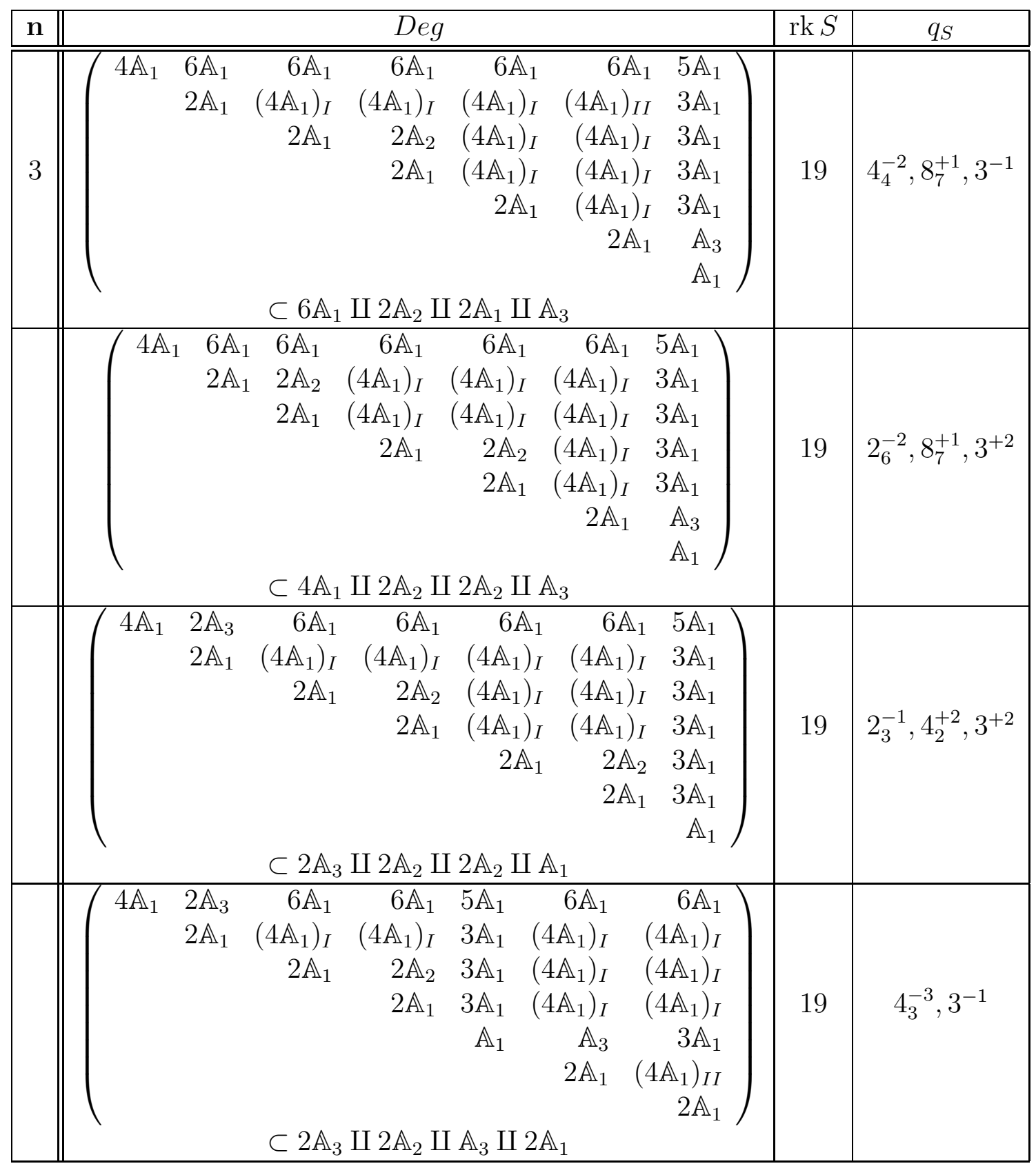




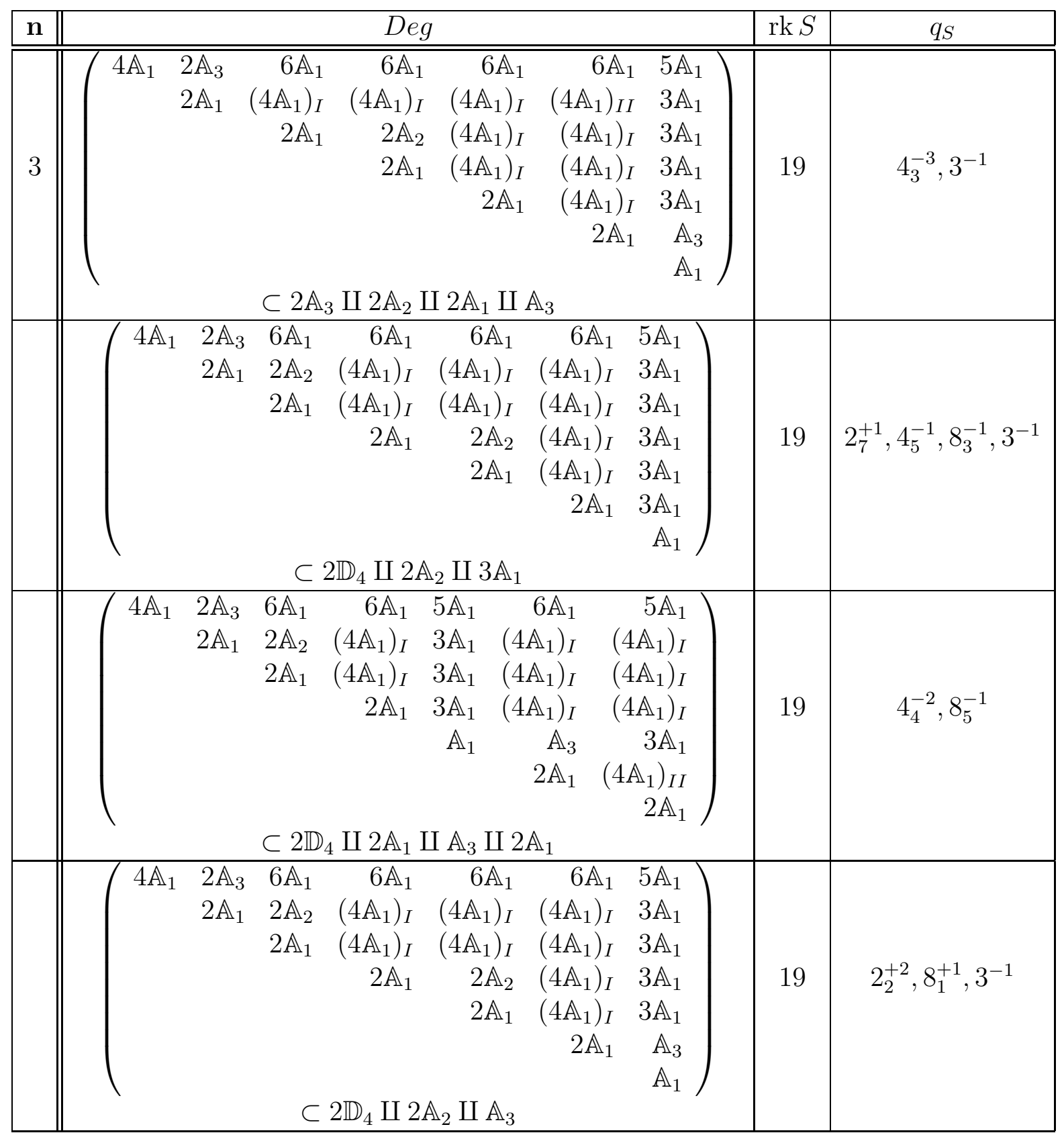




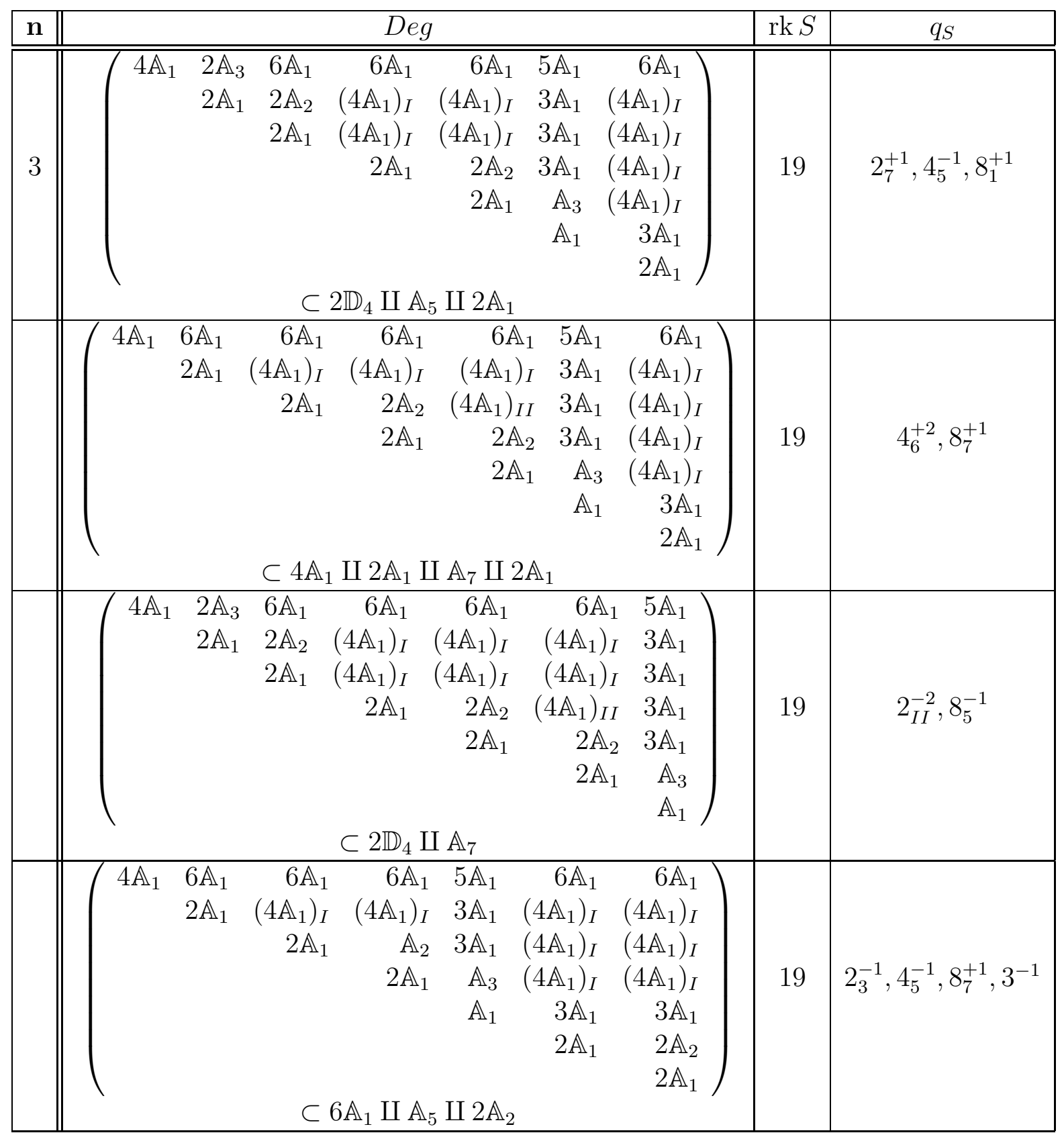




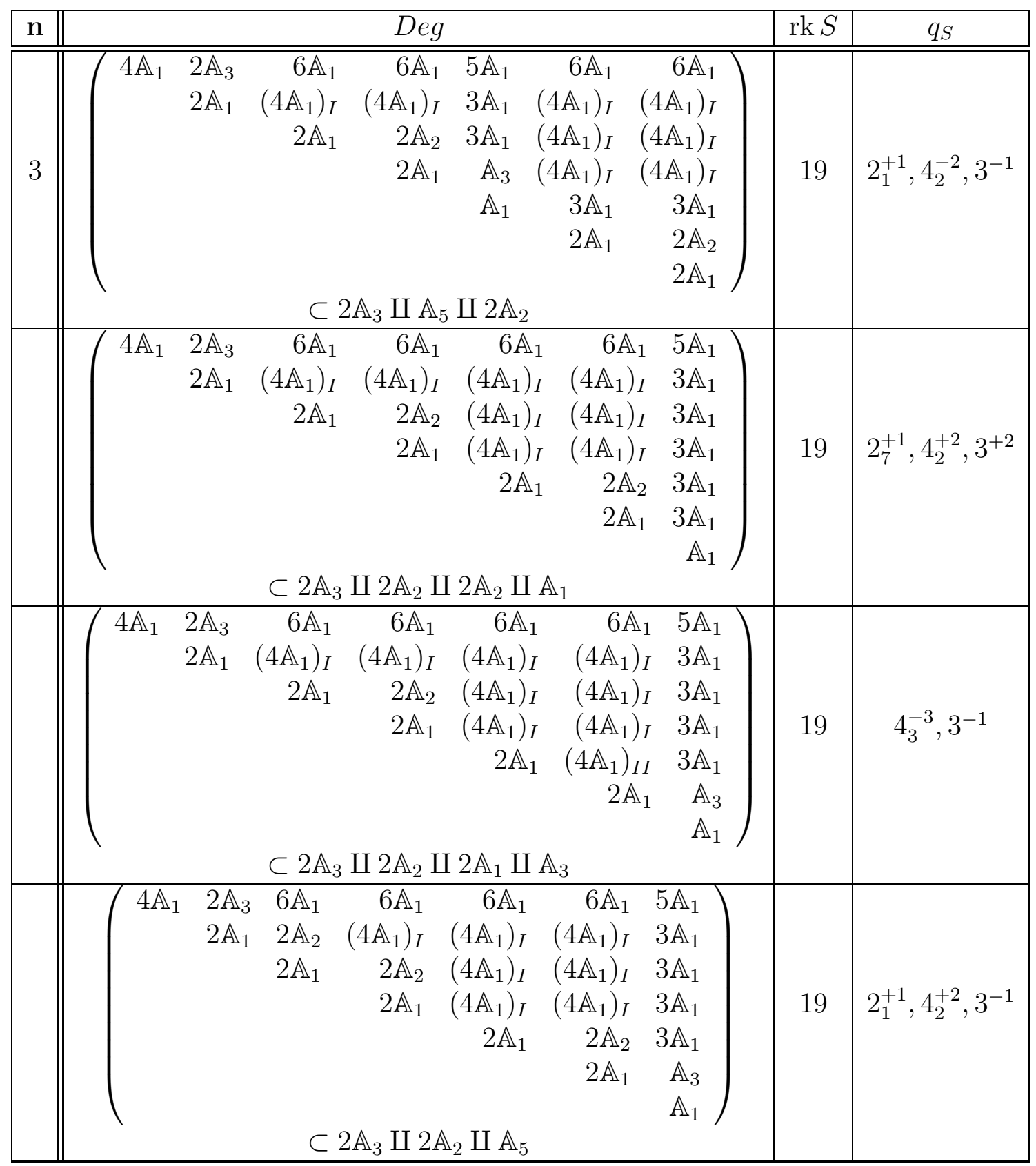




\begin{tabular}{|c|c|c|c|}
\hline n & $D e g$ & $\operatorname{rks} S$ & $q_{S}$ \\
\hline \multirow[t]{6}{*}{3} & 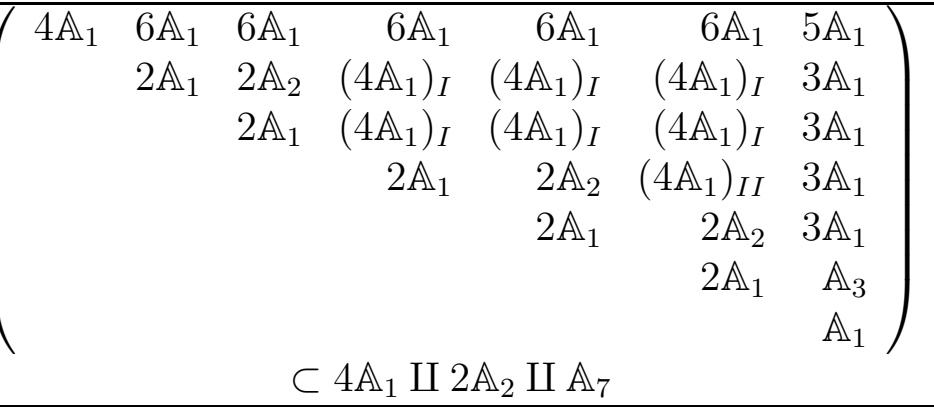 & 19 & $2_{I I}^{-2}, 8_{7}^{+1}, 3^{-1}$ \\
\hline & $\begin{array}{rrrrrrr}4 \mathbb{A}_{1} & 2 \mathbb{A}_{3} & 6 \mathbb{A}_{1} & 6 \mathbb{A}_{1} & 6 \mathbb{A}_{1} & 5 \mathbb{A}_{1} & 6 \mathbb{A}_{1} \\
& 2 \mathbb{A}_{1} & \left(4 \mathbb{A}_{1}\right)_{I} & \left(4 \mathbb{A}_{1}\right)_{I} & \left(4 \mathbb{A}_{1}\right)_{I} & 3 \mathbb{A}_{1} & \left(4 \mathbb{A}_{1}\right)_{I} \\
& & 2 \mathbb{A}_{1} & 2 \mathbb{A}_{2} & \left(4 \mathbb{A}_{1}\right)_{I I} & 3 \mathbb{A}_{1} & \left(4 \mathbb{A}_{1}\right)_{I} \\
& & 2 \mathbb{A}_{1} & 2 \mathbb{A}_{2} & 3 \mathbb{A}_{1} & \left(4 \mathbb{A}_{1}\right)_{I} \\
& & & 2 \mathbb{A}_{1} & \mathbb{A}_{3} & \left(4 \mathbb{A}_{1}\right)_{I} \\
& & & & \mathbb{A}_{1} & 3 \mathbb{A}_{1} \\
& & & & & & 2 \mathbb{A}_{1}\end{array}$ & 19 & $4_{5}^{+3}$ \\
\hline & $\begin{array}{c}\left(\mathbb{A}_{1},\left(2 \mathbb{A}_{1}, 2 \mathbb{A}_{1}\right)_{I}, 2 \mathbb{A}_{1}, 2 \mathbb{A}_{1}, 4 \mathbb{A}_{1}, 4 \mathbb{A}_{1}\right) \\
\subset 5 \mathbb{A}_{1} \amalg 2 \mathbb{A}_{2} \amalg 4 \mathbb{A}_{2}\end{array}$ & 19 & $2_{3}^{-1}, 4_{6}^{-2}, 3^{-2}$ \\
\hline & $\begin{array}{c}\left(\mathbb{A}_{1}, 2 \mathbb{A}_{1}, 2 \mathbb{A}_{1}, 2 \mathbb{A}_{1}, 2 \mathbb{A}_{1}, 4 \mathbb{A}_{1}, 4 \mathbb{A}_{1}\right) \\
\subset \mathbb{A}_{1} \amalg 2 \mathbb{A}_{2} \amalg 2 \mathbb{A}_{2} \amalg 4 \mathbb{A}_{2} \\
\end{array}$ & 19 & $2_{7}^{-3}, 3^{+3}$ \\
\hline & $\left(\begin{array}{ccccccc}4 \mathbb{A}_{1} & 4 \mathbb{A}_{2} & 6 \mathbb{A}_{1} & 6 \mathbb{A}_{1} & 6 \mathbb{A}_{1} & 6 \mathbb{A}_{1} & 5 \mathbb{A}_{1} \\
& 4 \mathbb{A}_{1} & 6 \mathbb{A}_{1} & 6 \mathbb{A}_{1} & 6 \mathbb{A}_{1} & 6 \mathbb{A}_{1} & 5 \mathbb{A}_{1} \\
& & 2 \mathbb{A}_{1} & 2 \mathbb{A}_{2} & \left(4 \mathbb{A}_{1}\right)_{I} & \left(4 \mathbb{A}_{1}\right)_{I} & 3 \mathbb{A}_{1} \\
& & & 2 \mathbb{A}_{1} & \left(4 \mathbb{A}_{1}\right)_{I} & \left(4 \mathbb{A}_{1}\right)_{I} & 3 \mathbb{A}_{1} \\
& & & & 2 \mathbb{A}_{1} & \left(4 \mathbb{A}_{1}\right)_{I} & 3 \mathbb{A}_{1} \\
& & & & & 2 \mathbb{A}_{1} & \mathbb{A}_{3} \\
& & & & & & \mathbb{A}_{1}\end{array}\right)$ & 19 & $2_{0}^{+2}, 4_{5}^{-1}, 3^{-2}$ \\
\hline & 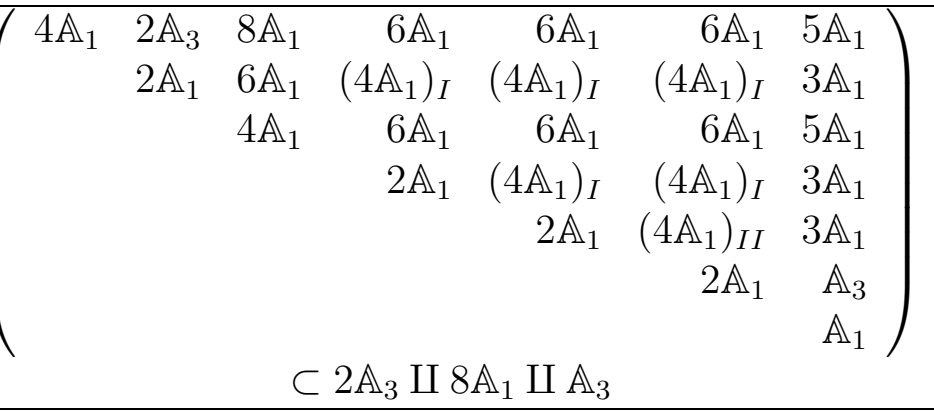 & 19 & $4_{4}^{-2}, 8_{5}^{-1}$ \\
\hline
\end{tabular}




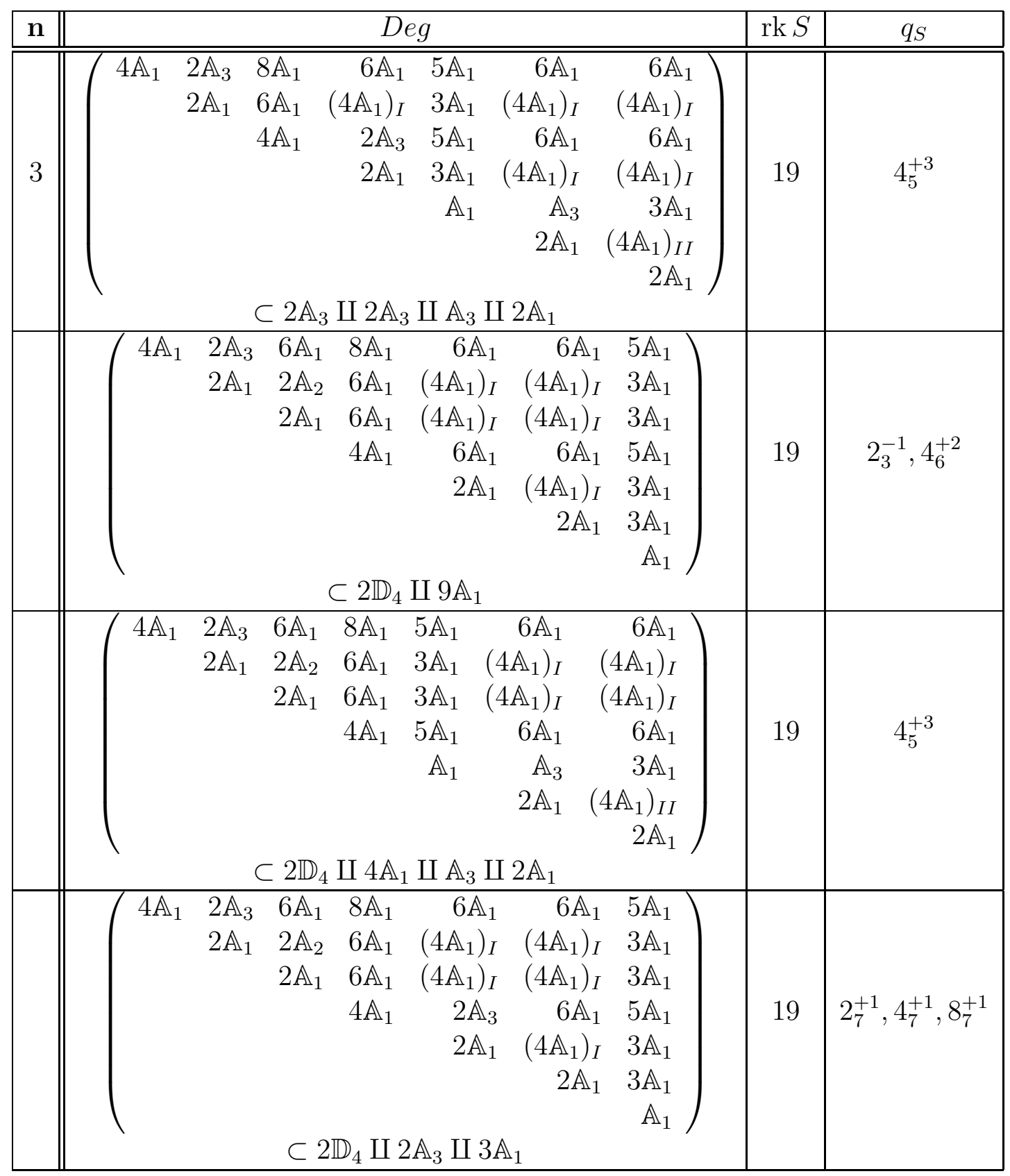




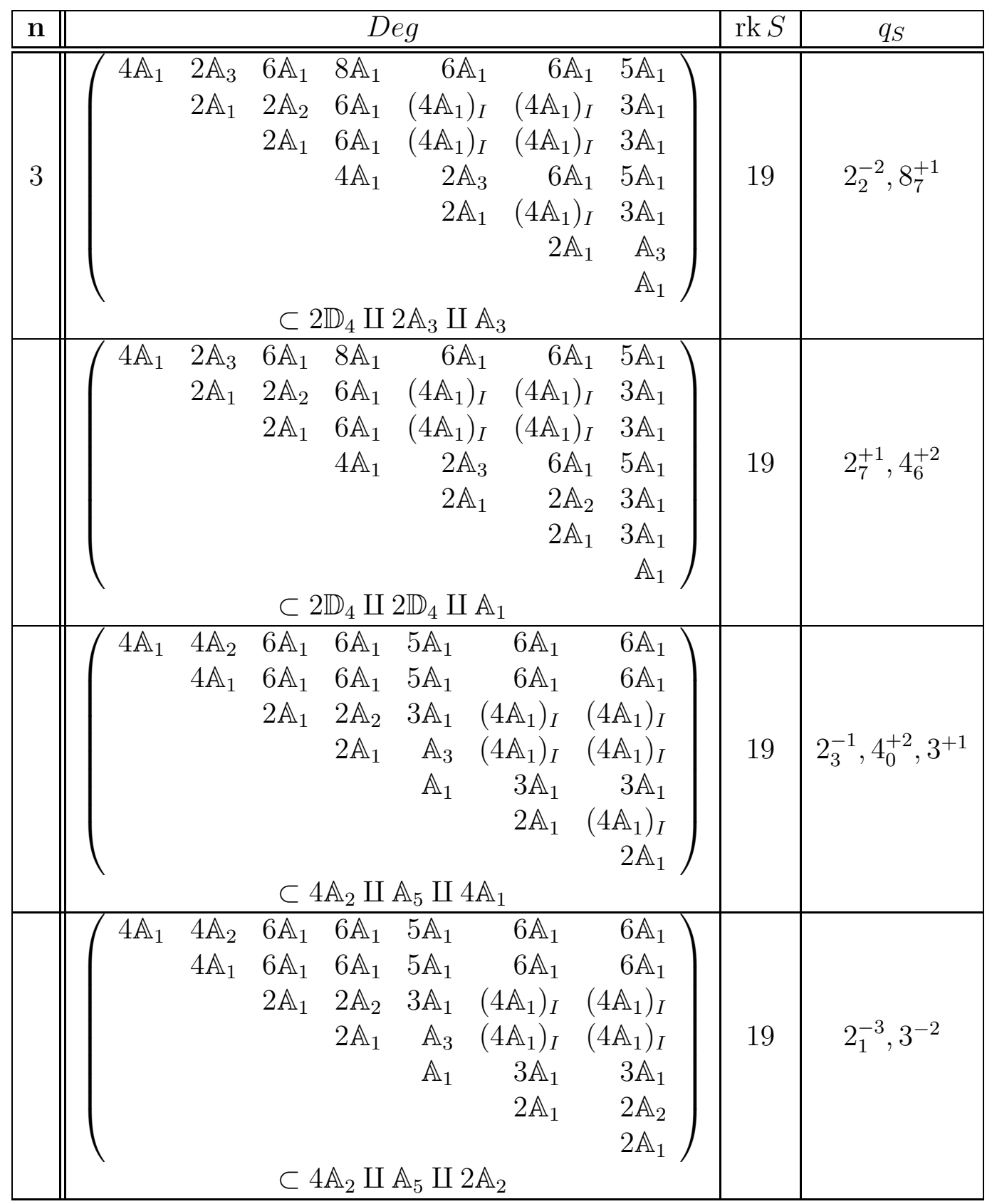




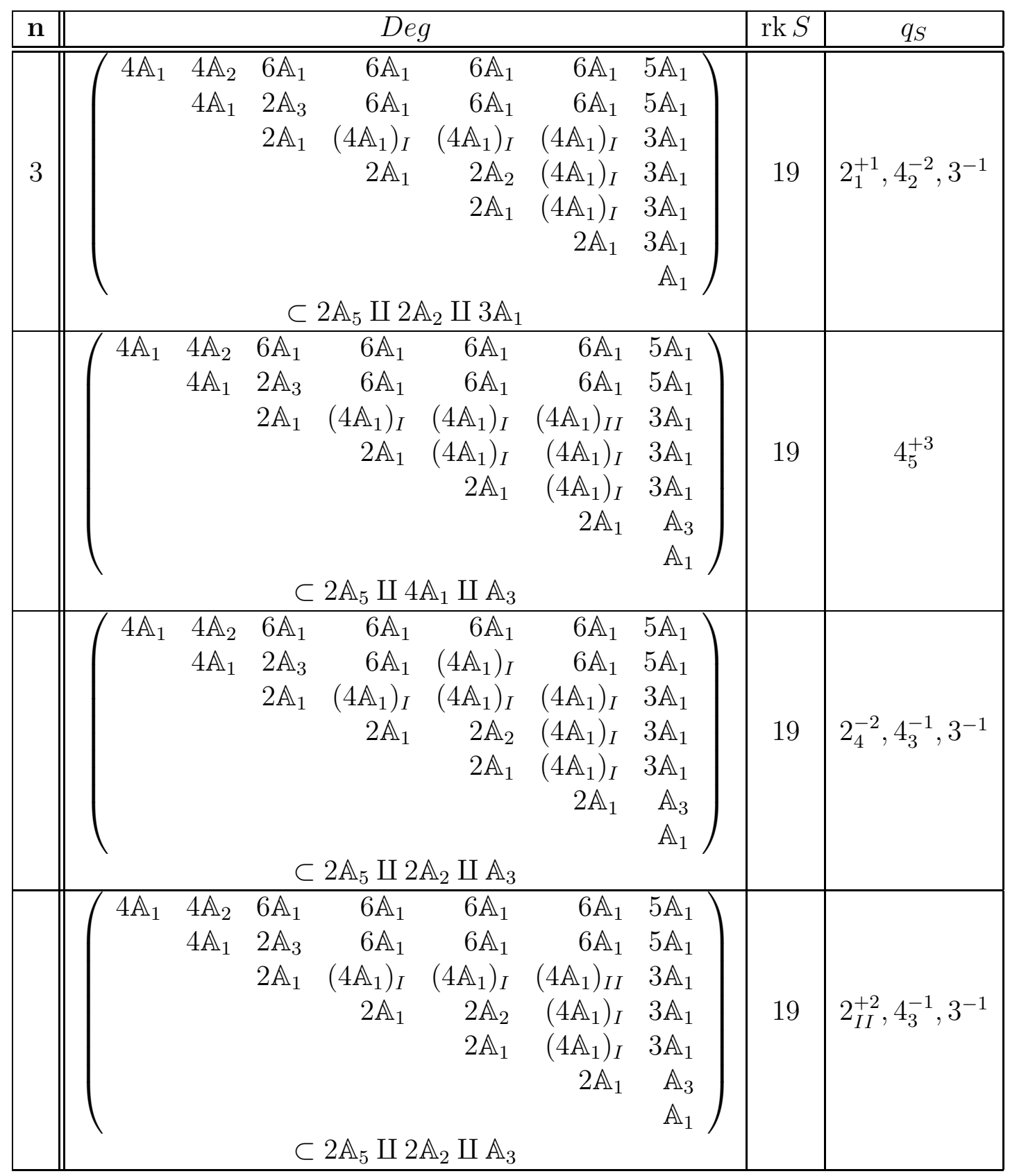




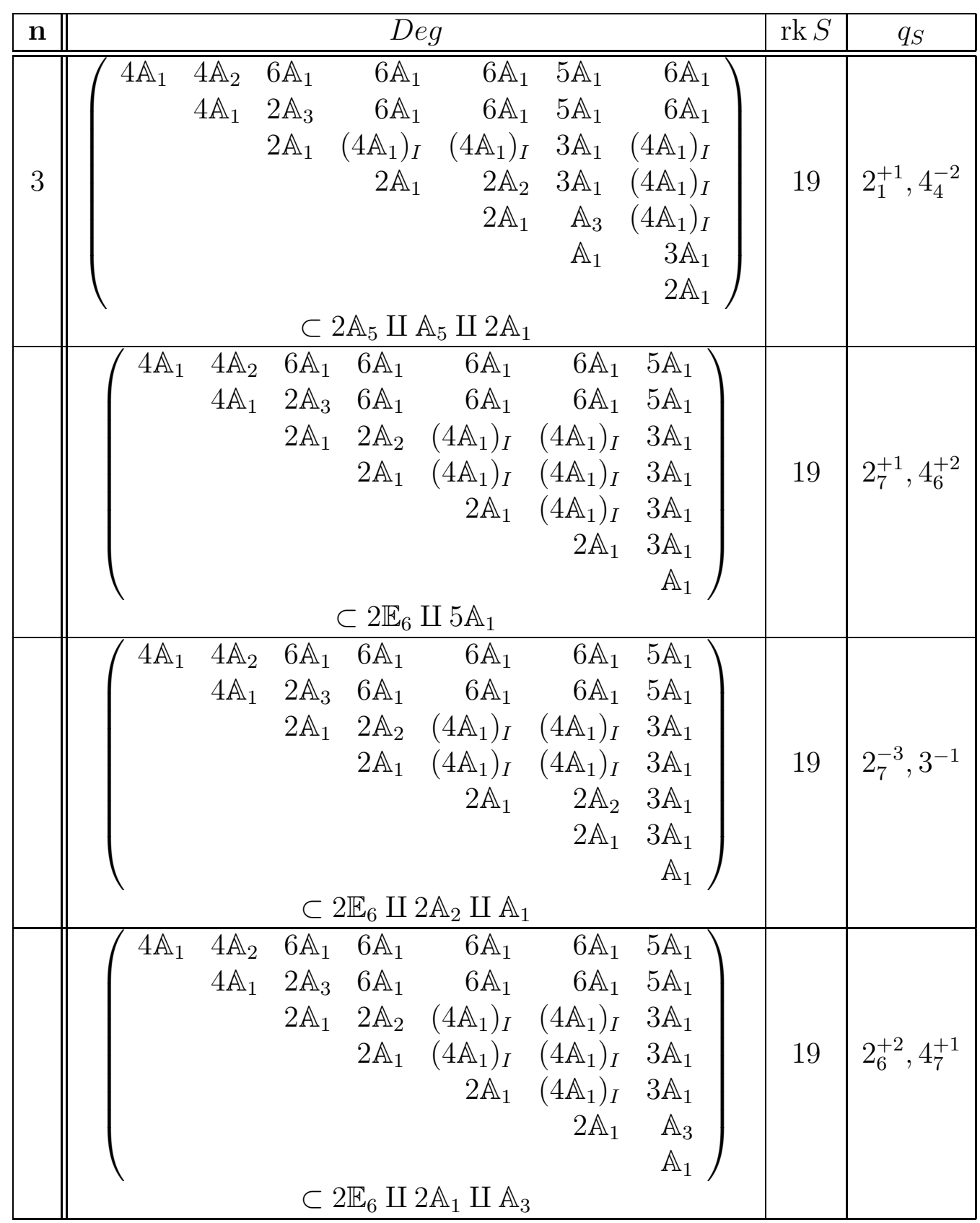




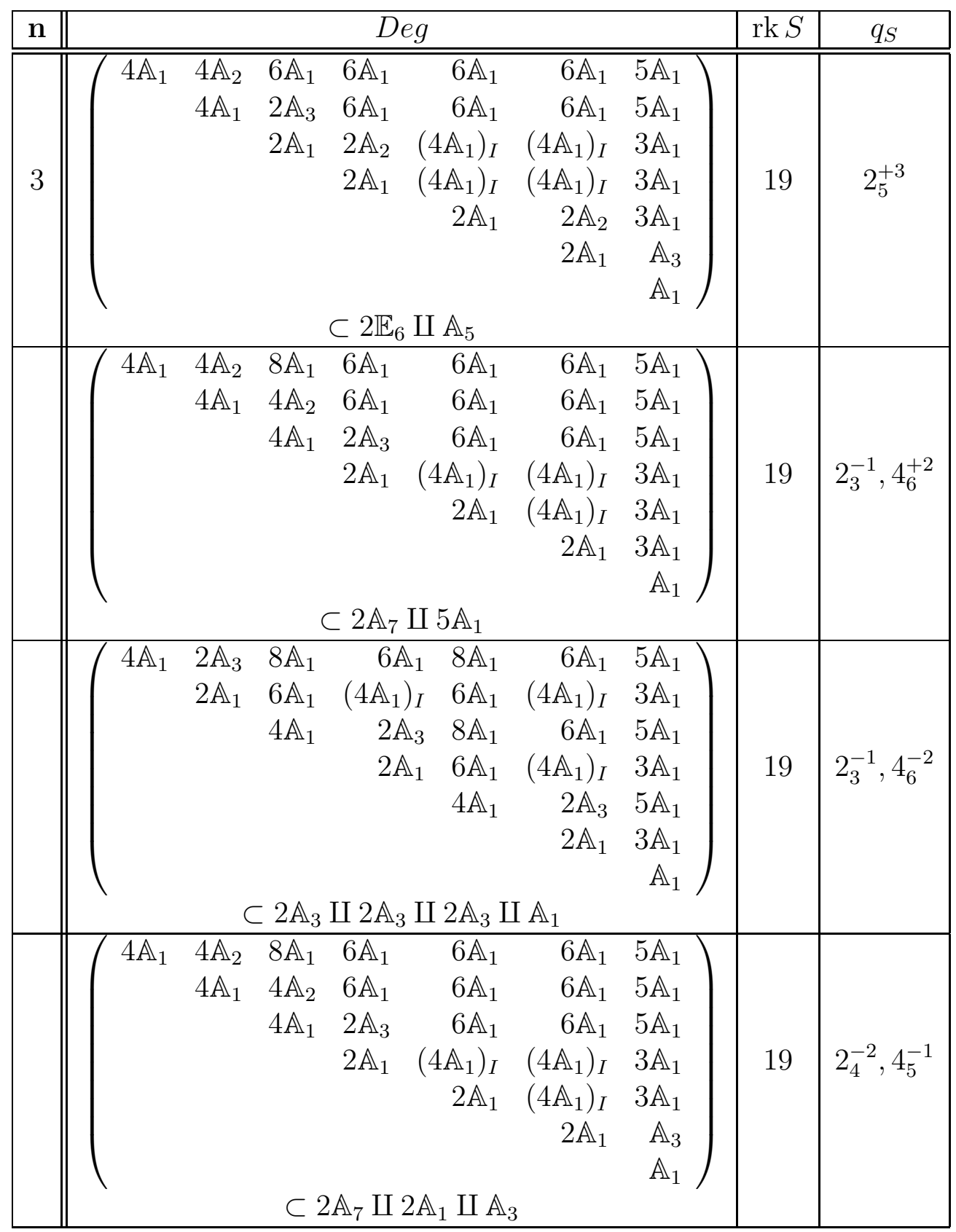




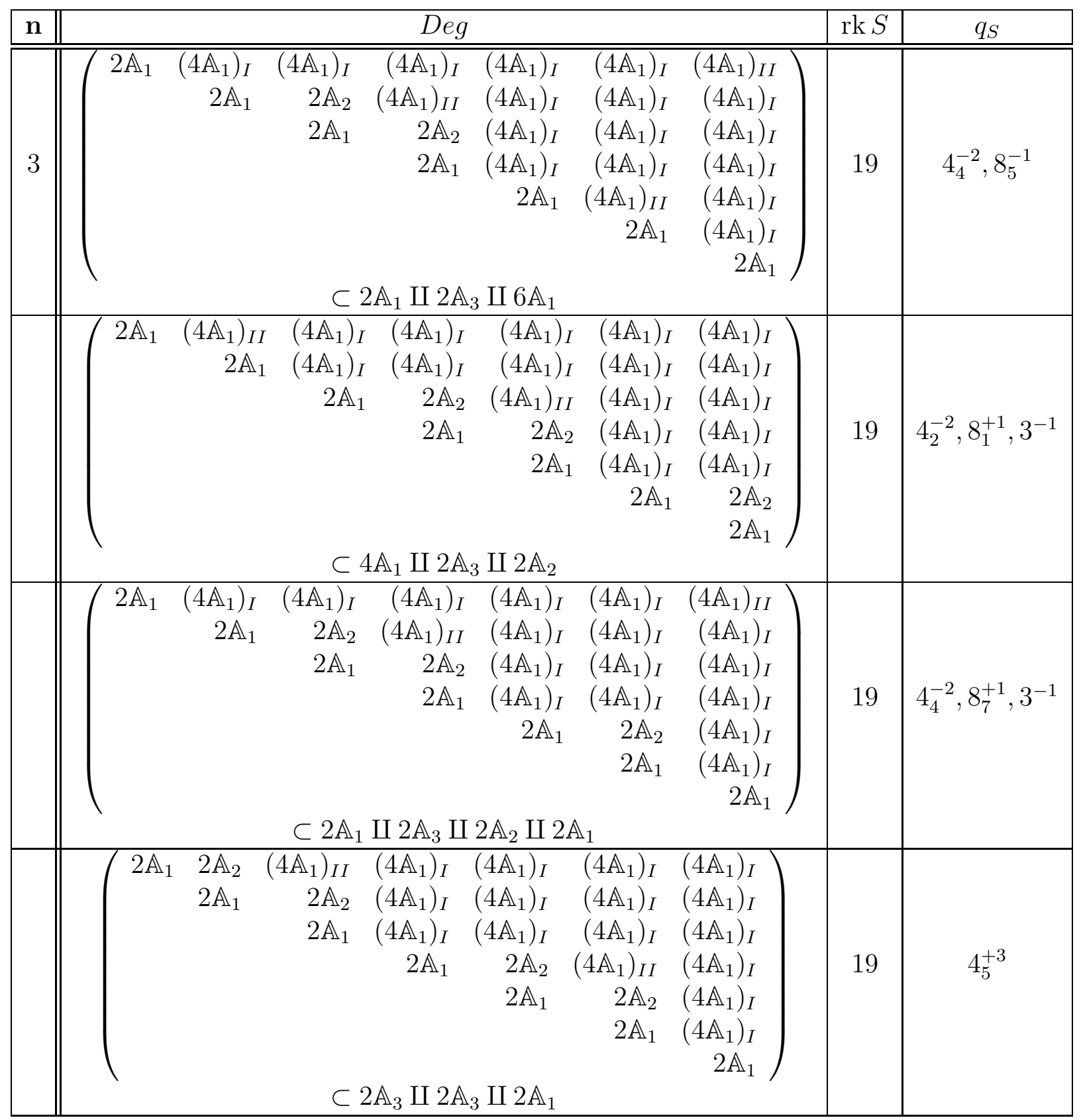




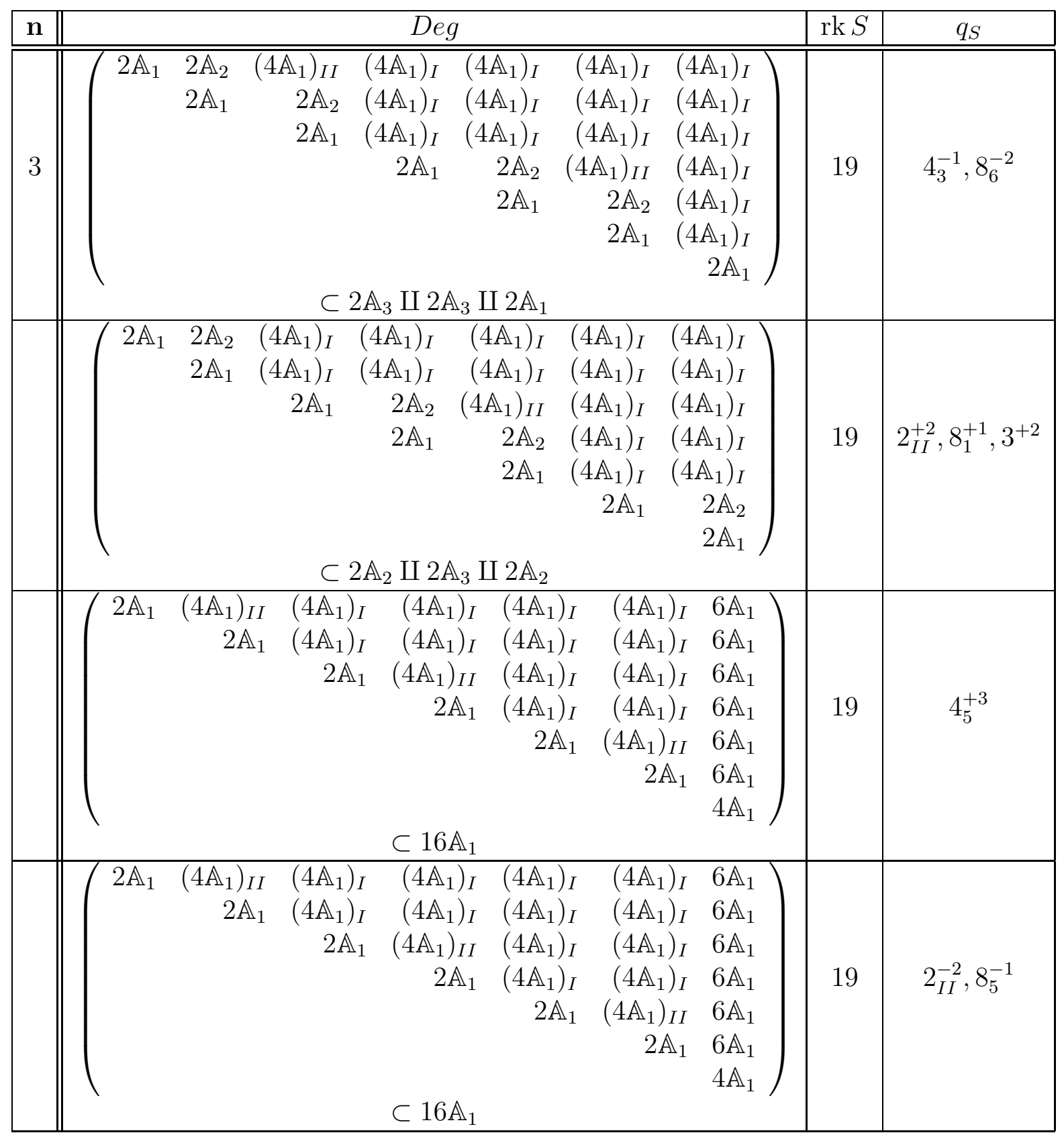




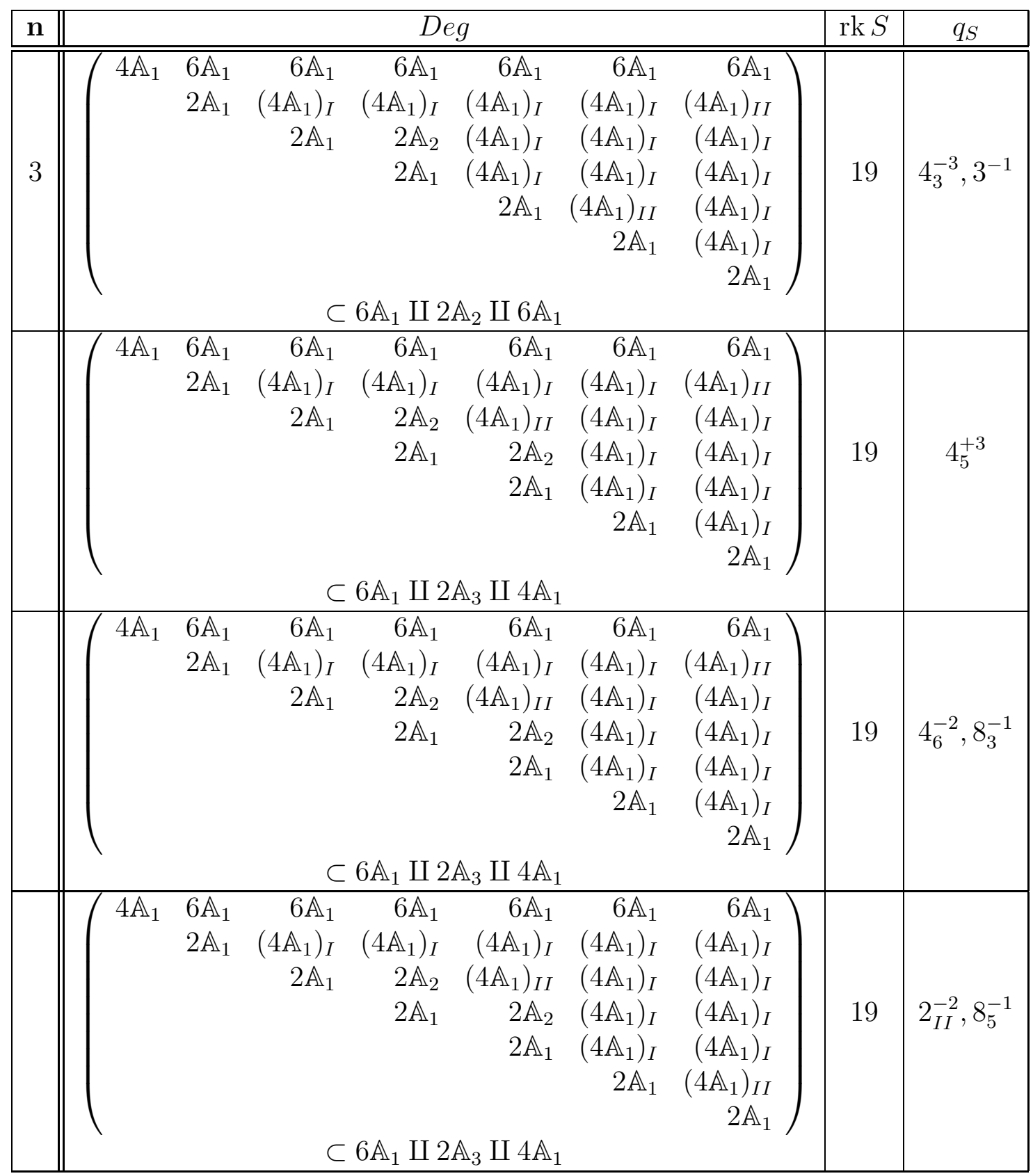




\begin{tabular}{|c|c|c|c|c|c|c|c|c|}
\hline n & \multicolumn{6}{|c|}{ Deg } & rk $S$ & $q_{S}$ \\
\hline 3 & $4 \mathbb{A}_{1}$ & $\begin{array}{l}6 \mathbb{A}_{1} \\
2 \mathbb{A}_{1}\end{array}$ & $\begin{array}{rr}6 \mathbb{A}_{1} & 6 \mathbb{A}_{1} \\
\left(4 \mathbb{A}_{1}\right)_{I} & \left(4 \mathbb{A}_{1}\right)_{I} \\
2 \mathbb{A}_{1} & 2 \mathbb{A}_{2} \\
& 2 \mathbb{A}_{1} \\
& \\
& \\
\subset 6 \mathbb{A}_{1} \amalg 2 \mathbb{A}_{2} \amalg\end{array}$ & $\begin{array}{r}6 \mathbb{A}_{1} \\
\left(4 \mathbb{A}_{1}\right)_{I} \\
\left(4 \mathbb{A}_{1}\right)_{I} \\
\left(4 \mathbb{A}_{1}\right)_{I} \\
2 \mathbb{A}_{1} \\
\\
\\
{\left[2 \mathbb{A}_{2} \amalg 2 .\right.}\end{array}$ & $\begin{array}{r}6 \mathbb{A}_{1} \\
\left(4 \mathbb{A}_{1}\right)_{I} \\
\left(4 \mathbb{A}_{1}\right)_{I} \\
\left(4 \mathbb{A}_{1}\right)_{I} \\
2 \mathbb{A}_{2} \\
2 \mathbb{A}_{1} \\
\\
\mathbb{A}_{1}\end{array}$ & $\left.\begin{array}{r}6 \mathbb{A}_{1} \\
\left(4 \mathbb{A}_{1}\right)_{I I} \\
\left(4 \mathbb{A}_{1}\right)_{I} \\
\left(4 \mathbb{A}_{1}\right)_{I} \\
\left(4 \mathbb{A}_{1}\right)_{I} \\
\left(4 \mathbb{A}_{1}\right)_{I} \\
2 \mathbb{A}_{1}\end{array}\right)$ & 19 & $2_{I I}^{+2}, 4_{1}^{+1}, 3^{+2}$ \\
\hline & $4 \mathbb{A}_{1}$ & $\begin{array}{l}6 \mathbb{A}_{1} \\
2 \mathbb{A}_{1}\end{array}$ & $\begin{array}{rr}6 \mathbb{A}_{1} & 6 \mathbb{A}_{1} \\
\left(4 \mathbb{A}_{1}\right)_{I} & \left(4 \mathbb{A}_{1}\right)_{I} \\
2 \mathbb{A}_{1} & 2 \mathbb{A}_{2} \\
& 2 \mathbb{A}_{1} \\
& \\
& \\
\subset 6 \mathbb{A}_{1} \amalg 2 \mathbb{A}_{2} \amalg\end{array}$ & $\begin{array}{r}6 \mathbb{A}_{1} \\
\left(4 \mathbb{A}_{1}\right)_{I} \\
\left(4 \mathbb{A}_{1}\right)_{I} \\
\left(4 \mathbb{A}_{1}\right)_{I} \\
2 \mathbb{A}_{1} \\
\\
\\
\\
2 \mathbb{A}_{2} \amalg 2 \\
\end{array}$ & $\begin{array}{r}6 \mathbb{A}_{1} \\
\left(4 \mathbb{A}_{1}\right)_{I} \\
\left(4 \mathbb{A}_{1}\right)_{I} \\
\left(4 \mathbb{A}_{1}\right)_{I} \\
2 \mathbb{A}_{2} \\
2 \mathbb{A}_{1} \\
\\
\mathbb{A}_{1}\end{array}$ & $\left.\begin{array}{r}6 \mathbb{A}_{1} \\
\left(4 \mathbb{A}_{1}\right)_{I I} \\
\left(4 \mathbb{A}_{1}\right)_{I} \\
\left(4 \mathbb{A}_{1}\right)_{I} \\
\left(4 \mathbb{A}_{1}\right)_{I} \\
\left(4 \mathbb{A}_{1}\right)_{I} \\
2 \mathbb{A}_{1}\end{array}\right)$ & 19 & $2_{I I}^{+2}, 8_{1}^{+1}, 3^{+2}$ \\
\hline & $4 \mathbb{A}_{1}$ & $\begin{array}{l}6 \mathbb{A}_{1} \\
2 \mathbb{A}_{1}\end{array}$ & $\begin{array}{rr}6 \mathbb{A}_{1} & 6 \mathbb{A}_{1} \\
\left(4 \mathbb{A}_{1}\right)_{I} & \left(4 \mathbb{A}_{1}\right)_{I} \\
2 \mathbb{A}_{1} & 2 \mathbb{A}_{2} \\
& 2 \mathbb{A}_{1} \\
& \\
& \\
\subset 6 \mathbb{A}_{1} \amalg 2 \mathbb{A}\end{array}$ & $\begin{array}{r}6 \mathbb{A}_{1} \\
\left(4 \mathbb{A}_{1}\right)_{I} \\
\left(4 \mathbb{A}_{1}\right)_{I I} \\
2 \mathbb{A}_{2} \\
2 \mathbb{A}_{1} \\
\\
\\
\\
\mathbb{A}_{3} \amalg 2 \mathbb{A}_{2}\end{array}$ & $\begin{array}{r}6 \mathbb{A}_{1} \\
\left(4 \mathbb{A}_{1}\right)_{I} \\
\left(4 \mathbb{A}_{1}\right)_{I} \\
\left(4 \mathbb{A}_{1}\right)_{I} \\
\left(4 \mathbb{A}_{1}\right)_{I} \\
2 \mathbb{A}_{1} \\
\end{array}$ & $\left.\begin{array}{r}6 \mathbb{A}_{1} \\
\left(4 \mathbb{A}_{1}\right)_{I} \\
\left(4 \mathbb{A}_{1}\right)_{I} \\
\left(4 \mathbb{A}_{1}\right)_{I} \\
\left(4 \mathbb{A}_{1}\right)_{I} \\
2 \mathbb{A}_{2} \\
2 \mathbb{A}_{1}\end{array}\right)$ & 19 & $4_{3}^{-3}, 3^{-1}$ \\
\hline & $4 \mathbb{A}_{1}$ & $\begin{array}{l}2 \mathbb{A}_{3} \\
2 \mathbb{A}_{1}\end{array}$ & $\begin{array}{rr}6 \mathbb{A}_{1} & 6 \mathbb{A}_{1} \\
\left(4 \mathbb{A}_{1}\right)_{I I} & \left(4 \mathbb{A}_{1}\right)_{I} \\
2 \mathbb{A}_{1} & \left(4 \mathbb{A}_{1}\right)_{I} \\
& 2 \mathbb{A}_{1} \\
& \\
& \\
\subset 2 \mathbb{A}_{3} \amalg 2 \mathbb{A}_{1} \amalg\end{array}$ & $\begin{array}{r}6 \mathbb{A}_{1} \\
\left(4 \mathbb{A}_{1}\right)_{I} \\
\left(4 \mathbb{A}_{1}\right)_{I} \\
2 \mathbb{A}_{2} \\
2 \mathbb{A}_{1} \\
\\
\\
2 \mathbb{A}_{2} \amalg 4 \\
\end{array}$ & $\begin{array}{r}6 \mathbb{A}_{1} \\
\left(4 \mathbb{A}_{1}\right)_{I} \\
\left(4 \mathbb{A}_{1}\right)_{I} \\
\left(4 \mathbb{A}_{1}\right)_{I} \\
\left(4 \mathbb{A}_{1}\right)_{I} \\
2 \mathbb{A}_{1} \\
\\
\mathbb{A}_{1} \\
\end{array}$ & $\begin{array}{r}6 \mathbb{A}_{1} \\
\left(4 \mathbb{A}_{1}\right)_{I} \\
\left(4 \mathbb{A}_{1}\right)_{I} \\
\left(4 \mathbb{A}_{1}\right)_{I} \\
\left(4 \mathbb{A}_{1}\right)_{I} \\
\left(4 \mathbb{A}_{1}\right)_{I I} \\
2 \mathbb{A}_{1}\end{array}$ & 19 & $4_{3}^{-3}, 3^{-1}$ \\
\hline
\end{tabular}




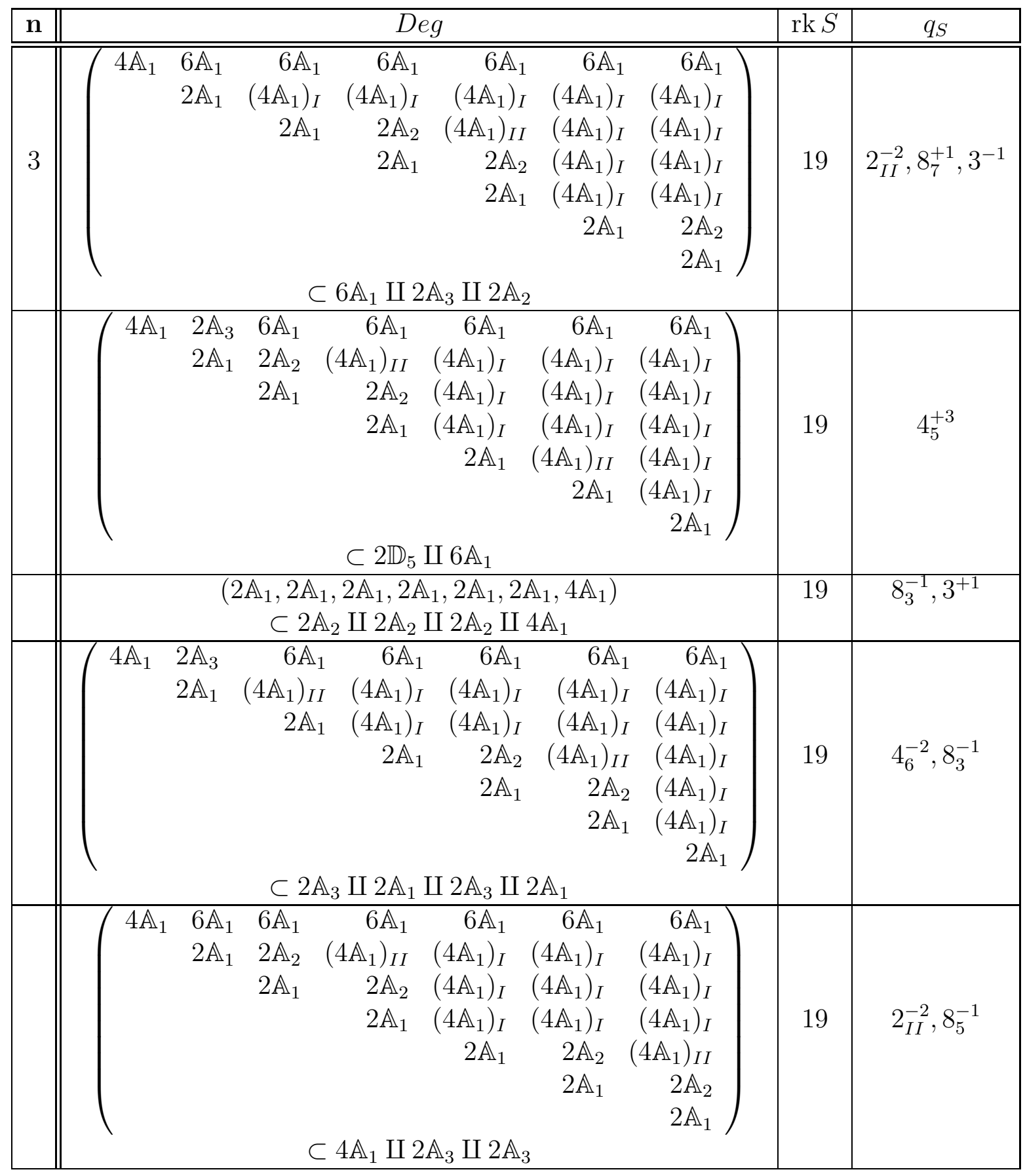




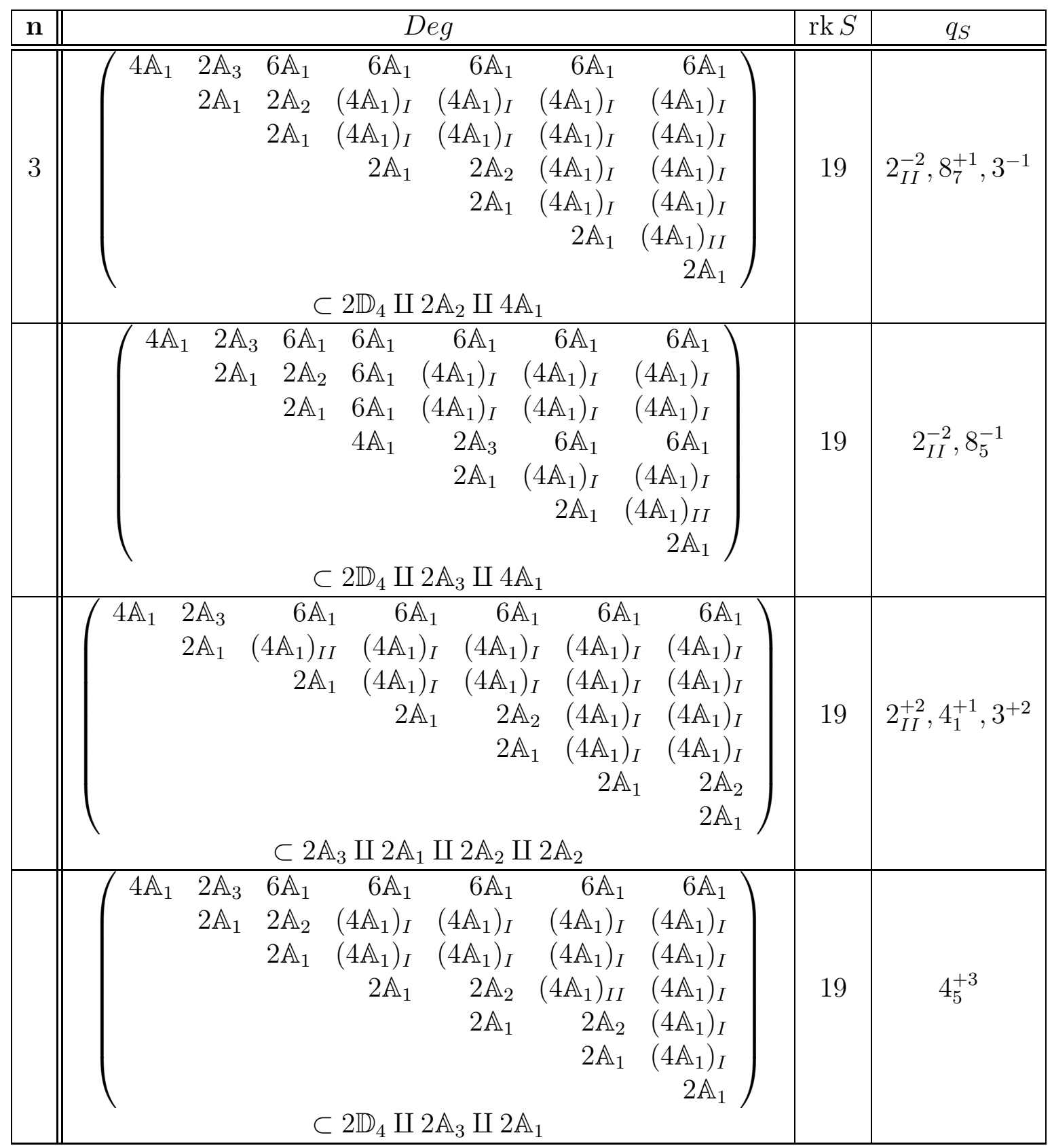




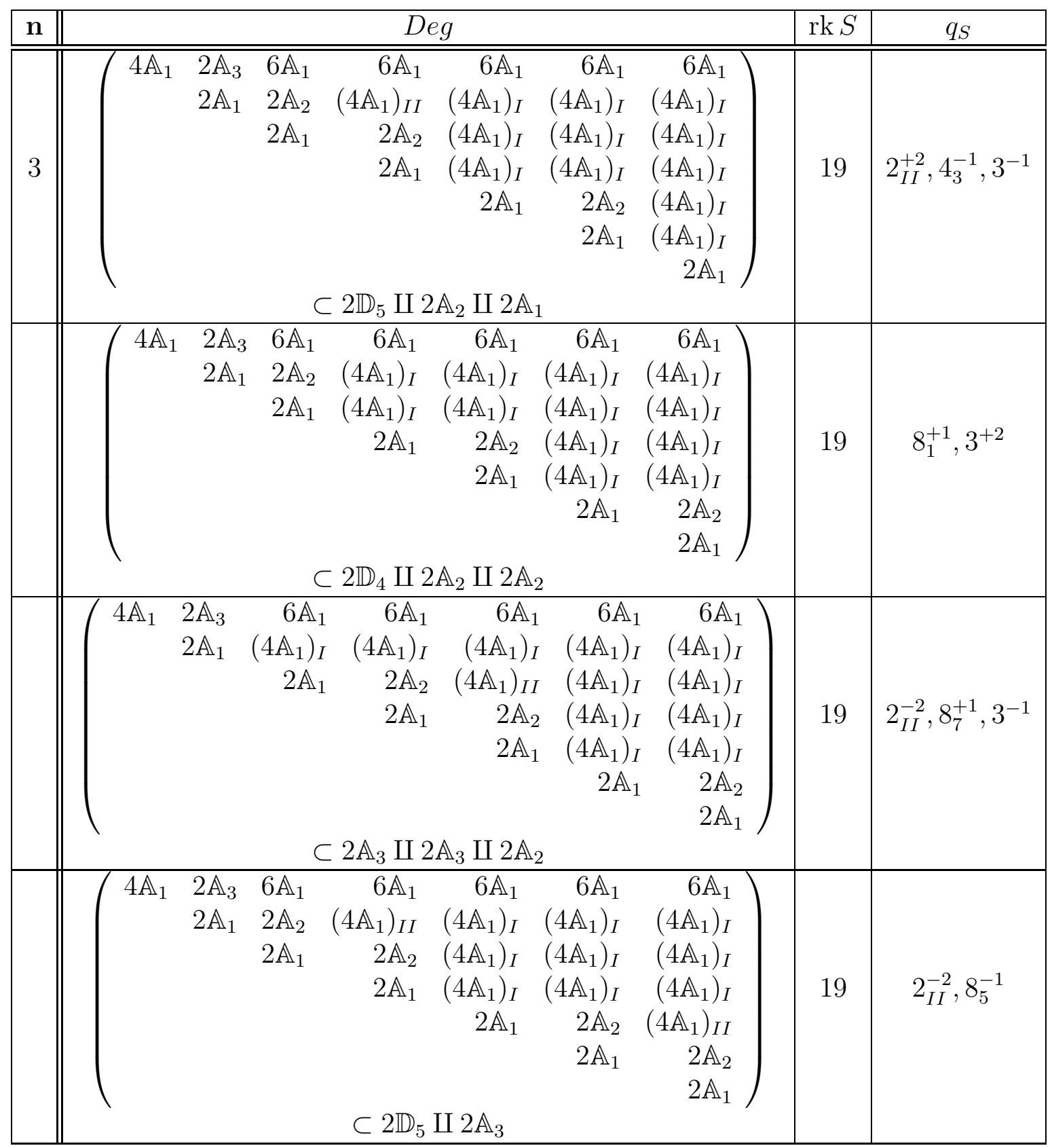




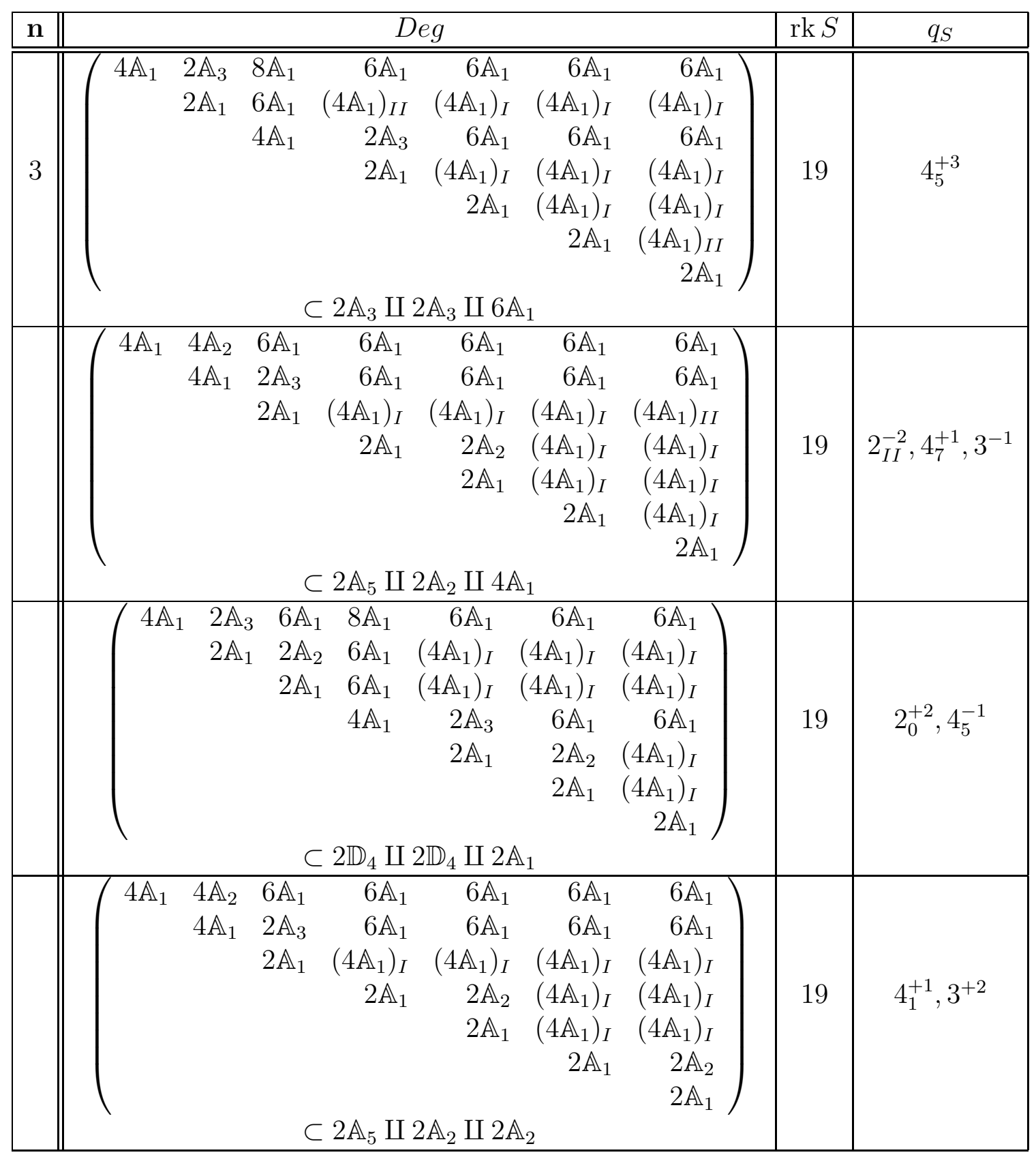




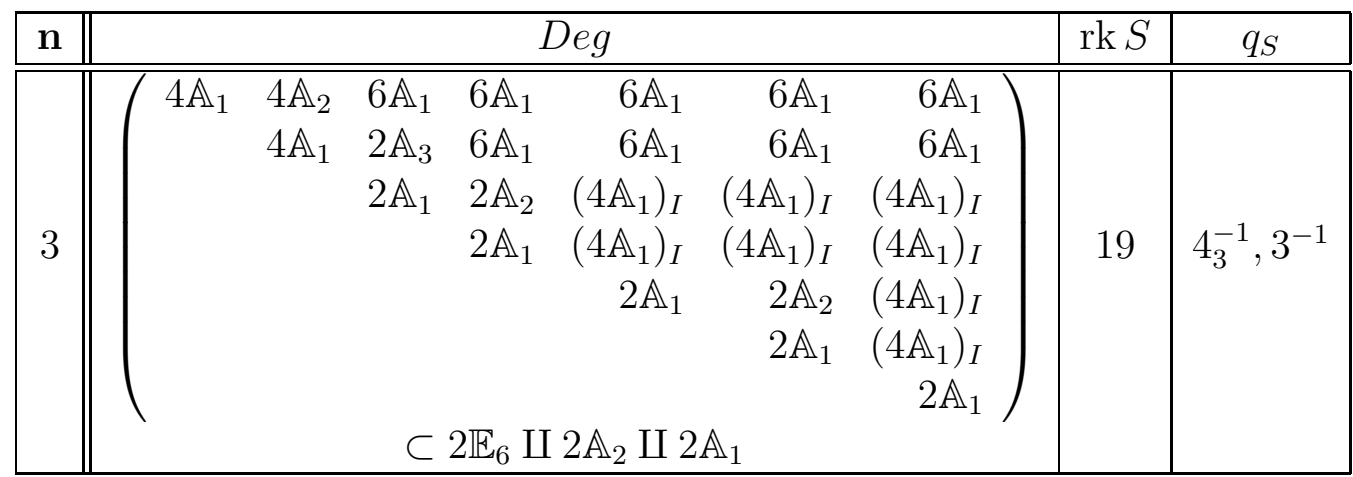

\section{References}

[1] N. Bourbaki, Groupes et algèbres de Lie, Groupes de Coxeter et systèmes de Tits, Groupes engendres par des reflexions, systèmes de racines, Hermann, Paris VI, 1968.

[2] D. Burns, M. Rapoport, On the Torelli problem for Kählerian K-3 surfaces, Ann. scient. Éc. Norm. Sup. $4^{e}$ ser. 8 (1975), 235-274.

[3] J.H. Conway, N.J.A. Sloane, Sphere packings, lattices and groups, Grundlehren Math. Wiss., 290, Springer-Verlag, New York, 1988. 663 pages.

[4] The GAP Group, GAP-Groups, Algorithms and Programming, Version 4.6.5, 2013, http://www.gap-system.org

[5] K. Hashimoto, Finite symplectic actions on the K3 lattice, Nagoya Math J., 206 (2012), 99-153 (see also arXiv:1012.2682).

[6] Sh. Kondō, Niemeier lattices, Mathieu groups, and finite groups of symplectic automorphisms of K3 surfaces (with appendix by Sh. Mukai), Duke Math. J. 92, (1998), 593-603.

[7] Vic. S. Kulikov, Degenerations of K3 surfaces and Enriques surfaces, Izv. Akad. Nauk SSSR Ser. Mat. 41 (1977), no. 5, 1008-1042; English transl. in Math. USSR Izv. 11, (1977) no. 5, 957-989.

[8] Sh. Mukai, Finite groups of automorphisms of K3 surfaces and the Mathieu group, Invent. math. 94, (1988), 183-221.

[9] V.V. Nikulin, On Kummer surfaces, Izv. Akad. Nauk SSSR Ser. Mat. 39 (1975), no. 2, 278-293; English transl. in Math. USSR Izv. 9 (1975), 261-275. 
Table 2: Types and lattices $S$ of degenerations of codimension 1 of Kählerian K3 surfaces with finite symplectic automorphism groups $G=\operatorname{Clos}(G)$ (from [16]).

\begin{tabular}{|c|c|c|c|c|c|c|c|c|}
\hline $\mathbf{n}$ & $|G|$ & $i$ & $G$ & $\mathrm{rk} S_{G}$ & $q_{S_{S_{G}}}$ & Deg & rk $S$ & $q_{S}$ \\
\hline \multirow[t]{2}{*}{1} & 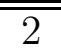 & 1 & $\overline{\overline{C_{2}}}$ & 88 & 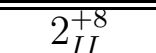 & 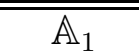 & $\overline{99}$ & $2_{7}^{+9}$ \\
\hline & & & & & & $2 \mathbb{A}_{1}$ & 9 & $2_{I I}^{-6}, 4_{3}^{-1}$ \\
\hline \multirow[t]{2}{*}{2} & 3 & 1 & $C_{3}$ & $\overline{12}$ & $3^{+6}$ & $\overline{\mathbb{A}_{1}}$ & 13 & $2_{3}^{-1}, 3^{+6}$ \\
\hline & & & & & & $3 \mathbb{A}_{1}$ & 13 & $2_{1}^{+1}, 3^{-5}$ \\
\hline \multirow[t]{3}{*}{3} & 4 & 2 & $C_{2}^{2}$ & 12 & $2_{I I}^{-6}, 4_{I I}^{-2}$ & $\mathbb{A}_{1}$ & 13 & $2_{3}^{+7}, 4_{I I}^{+2}$ \\
\hline & & & & & & $2 \mathbb{A}_{1}$ & 13 & $2_{I I}^{-4}, 4_{7}^{-3}$ \\
\hline & & & & & & $4 \mathbb{A}_{1}$ & 13 & $2_{I I}^{-6}, 8_{3}^{-1}$ \\
\hline \multirow[t]{4}{*}{4} & 4 & 1 & $C_{4}$ & 14 & $2_{2}^{+2}, 4_{I I}^{+4}$ & 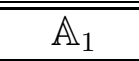 & 15 & 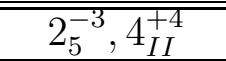 \\
\hline & & & & & & $2 \mathbb{A}_{1}$ & 15 & $4_{1}^{-5}$ \\
\hline & & & & & & $4 \mathbb{A}_{1}$ & 15 & $2_{2}^{+2}, 4_{I I}^{+2}, 8_{7}^{+1}$ \\
\hline & & & & & & $\mathbb{A}_{2}$ & 15 & $2_{1}^{+1}, 4_{I I}^{-4}$ \\
\hline \multirow[t]{4}{*}{6} & 6 & 1 & $D_{6}$ & 14 & $2_{I I}^{-2}, 3^{+5}$ & $\overline{\mathbb{A}_{1}}$ & 15 & $2_{7}^{-3}, 3^{+5}$ \\
\hline & & & & & & $2 \mathbb{A}_{1}$ & 15 & $4_{3}^{-1}, 3^{+5}$ \\
\hline & & & & & & $3 \mathbb{A}_{1}$ & 15 & $2_{1}^{-3}, 3^{-4}$ \\
\hline & & & & & & $6 \mathbb{A}_{1}$ & 15 & $4_{1}^{+1}, 3^{+4}$ \\
\hline \multirow[t]{3}{*}{9} & 8 & 5 & $C_{2}^{3}$ & 14 & $2_{I I}^{+6}, 4_{2}^{+2}$ & $2 \mathbb{A}_{1}$ & 15 & $2_{I I}^{-4}, 4_{5}^{-3}$ \\
\hline & & & & & & $4 \mathbb{A}_{1}$ & 15 & $2_{I I}^{+6}, 8_{1}^{+1}$ \\
\hline & & & & & & $8 \mathbb{A}_{1}$ & 15 & $2_{I I}^{+6}, 4_{1}^{+1}$ \\
\hline \multirow[t]{6}{*}{10} & 8 & 3 & $D_{8}$ & 15 & $4_{1}^{+5}$ & $\overline{\mathbb{A}_{1}}$ & $\overline{16}$ & 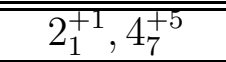 \\
\hline & & & & & & $\left(2 \mathbb{A}_{1}\right)_{I}$ & 16 & $2_{6}^{-2}, 4_{6}^{-4}$ \\
\hline & & & & & & $\left(2 \mathbb{A}_{1}\right)_{I I}$ & 16 & $2_{I I}^{+2}, 4_{I I}^{+4}$ \\
\hline & & & & & & $4 \mathbb{A}_{1}$ & 16 & $4_{7}^{+3}, 8_{1}^{+1}$ \\
\hline & & & & & & $8 \mathbb{A}_{1}$ & 16 & $4_{0}^{+4}$ \\
\hline & & & & & & $2 \mathbb{A}_{2}$ & 16 & $4_{I I}^{+4}$ \\
\hline \multirow[t]{2}{*}{12} & 8 & 4 & $Q_{8}$ & 17 & $22_{7}^{-3}, 8_{I I}^{-2}$ & $8 \mathbb{A}_{1}$ & 18 & $2_{7}^{-3}, 16_{3}^{-1}$ \\
\hline & & & & & & $\mathbb{A}_{2}$ & 18 & $2_{6}^{-2}, 8_{I I}^{-2}$ \\
\hline \multirow[t]{2}{*}{16} & 10 & 1 & $D_{10}$ & 16 & $5^{+4}$ & $\overline{\mathbb{A}_{1}}$ & $\overline{17}$ & $2_{7}^{+1}, 5^{+4}$ \\
\hline & & & & & & $5 \mathbb{A}_{1}$ & 17 & $2_{7}^{+1}, 5^{-3}$ \\
\hline
\end{tabular}




\begin{tabular}{|c|c|c|c|c|c|c|c|c|}
\hline $\mathbf{n}$ & $|G|$ & $i$ & $G$ & rk $S_{G}$ & $q_{S_{G}}$ & $D e g$ & rk $S$ & $q_{S}$ \\
\hline \multirow[t]{5}{*}{$\overline{17}$} & $\overline{12}$ & 3 & $\overline{\mathfrak{A}_{4}}$ & 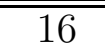 & $\overline{2}_{I I}^{-2}, 4_{I I}^{-2}, 3^{+2}$ & $\overline{\mathbb{A}_{1}}$ & $\overline{c 17}$ & $\bar{c}_{7}^{-3}, 4_{I I}^{+2}, 3^{+2}$ \\
\hline & & & & & & $3 \mathbb{A}_{1}$ & 17 & $2_{1}^{-3}, 4_{I I}^{+2}, 3^{-1}$ \\
\hline & & & & & & $4 \mathbb{A}_{1}$ & 17 & $2_{I I}^{-2}, 8_{3}^{-1}, 3^{+2}$ \\
\hline & & & & & & $6 \mathbb{A}_{1}$ & 17 & $4_{1}^{-3}, 3^{+1}$ \\
\hline & & & & & & $12 \mathbb{A}_{1}$ & 17 & $2_{I I}^{-2}, 8_{1}^{+1}, 3^{-1}$ \\
\hline \multirow[t]{4}{*}{18} & 12 & 4 & $D_{12}$ & 16 & $2_{I I}^{+4}, 3^{+4}$ & $\overline{\mathbb{A}_{1}}$ & $\overline{17}$ & $2_{7}^{+5}, 3^{+4}$ \\
\hline & & & & & & $2 \mathbb{A}_{1}$ & 17 & $2_{I I}^{+2}, 4_{7}^{+1}, 3^{+4}$ \\
\hline & & & & & & $3 \mathbb{A}_{1}$ & 17 & $2_{5}^{-5}, 3^{-3}$ \\
\hline & & & & & & $6 \mathbb{A}_{1}$ & 17 & $2_{I I}^{-2}, 4_{1}^{+1}, 3^{+3}$ \\
\hline \multirow[t]{2}{*}{21} & 16 & 14 & $C_{2}^{4}$ & 15 & $2_{I I}^{+6}, 8_{I}^{+1}$ & $4 \mathbb{A}_{1}$ & 16 & $2_{I I}^{+4}, 4_{I I}^{+2}$ \\
\hline & & & & & & $16 \mathbb{A}_{1}$ & 16 & $2_{I I}^{+6}$ \\
\hline \multirow[t]{3}{*}{22} & 16 & 11 & $\overline{C_{2} \times D_{8}}$ & 16 & $2_{I I}^{+2}, 4_{0}^{+4}$ & $2 \mathbb{A}_{1}$ & $\overline{17}$ & $4_{7}^{+5}$ \\
\hline & & & & & & $4 \mathbb{A}_{1}$ & 17 & $2_{I I}^{+2}, 4_{0}^{+2}, 8_{7}^{+1}$ \\
\hline & & & & & & $8 \mathbb{A}_{1}$ & 17 & $2_{I I}^{+2}, 4_{7}^{+3}$ \\
\hline \multirow[t]{2}{*}{26} & 16 & 8 & $S D_{16}$ & 18 & $2_{7}^{+1}, 4_{7}^{+1}, 8_{I I}^{+2}$ & $8 \mathbb{A}_{1}$ & $\overline{19}$ & $\overline{2}_{1}^{+1}, 4_{1}^{+1}, 16_{3}^{-1}$ \\
\hline & & & & & & $2 \mathbb{A}_{2}$ & 19 & $2_{5}^{-1}, 8_{I I}^{-2}$ \\
\hline \multirow[t]{2}{*}{30} & 18 & 4 & $\mathfrak{A}_{3,3}$ & 16 & $3^{+4}, 9^{-1}$ & $\overline{3 \mathbb{A}_{1}}$ & $\bar{~} 17$ & 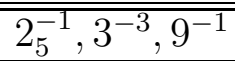 \\
\hline & & & & & & $9 \mathbb{A}_{1}$ & 17 & $2_{3}^{-1}, 3^{+4}$ \\
\hline \multirow[t]{4}{*}{32} & 20 & 3 & $\mathrm{Hol}\left(C_{5}\right)$ & 18 & $2_{6}^{-2}, 5^{+3}$ & $\overline{2 \mathbb{A}_{1}}$ & 19 & $\overline{4}_{1}^{+1}, 5^{+3}$ \\
\hline & & & & & & $5 \mathbb{A}_{1}$ & 19 & $2_{1}^{+3}, 5^{-2}$ \\
\hline & & & & & & $10 \mathbb{A}_{1}$ & 19 & $4_{5}^{-1}, 5^{+2}$ \\
\hline & & & & & & $5 \mathbb{A}_{2}$ & 19 & $2_{5}^{-1}, 5^{-2}$ \\
\hline 33 & 21 & 1 & $C_{7} \rtimes C_{3}$ & 18 & $7^{+3}$ & $\overline{7 \mathbb{A}_{1}}$ & $\overline{19}$ & $\overline{2}_{1}^{+1}, 7^{+2}$ \\
\hline \multirow[t]{9}{*}{34} & 24 & $\overline{12}$ & $\mathfrak{S}_{4}$ & 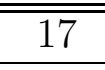 & $\overline{4_{3}^{+3}, 3^{+2}}$ & $\overline{\mathbb{A}_{1}}$ & 18 & $2_{5}^{-1}, 4_{1}^{+3}, 3^{+2}$ \\
\hline & & & & & & $2 \mathbb{A}_{1}$ & 18 & $2_{2}^{+2}, 4_{I I}^{+2}, 3^{+2}$ \\
\hline & & & & & & $3 \mathbb{A}_{1}$ & 18 & $2_{7}^{+1}, 4_{5}^{-3}, 3^{-1}$ \\
\hline & & & & & & $4 \mathbb{A}_{1}$ & 18 & $4_{3}^{-1}, 8_{3}^{-1}, 3^{+2}$ \\
\hline & & & & & & $\left(6 \mathbb{A}_{1}\right)_{I}$ & 18 & $2_{4}^{-2}, 4_{0}^{+2}, 3^{+1}$ \\
\hline & & & & & & $\left(6 \mathbb{A}_{1}\right)_{I I}$ & 18 & $2_{I I}^{+2}, 4_{I I}^{-2}, 3^{+1}$ \\
\hline & & & & & & $8 \mathbb{A}_{1}$ & 18 & $4_{2}^{+2}, 3^{+2}$ \\
\hline & & & & & & $12 \mathbb{A}_{1}$ & 18 & $4_{5}^{-1}, 8_{7}^{+1}, 3^{-1}$ \\
\hline & & & & & & $6 \mathbb{A}_{2}$ & 18 & $4_{I}^{-2}, 3^{+1}$ \\
\hline
\end{tabular}




\begin{tabular}{|c|c|c|c|c|c|c|c|c|}
\hline $\mathbf{n}$ & $|G|$ & $i$ & $G$ & $\operatorname{rk} S_{G}$ & $q_{S_{G}}$ & $D e g$ & rk $S$ & $q_{S}$ \\
\hline \multirow[t]{3}{*}{$\overline{39}$} & "32 & $\overline{\overline{27}}$ & $\bar{~} \overline{2^{4} C_{2}}$ & " 17 & 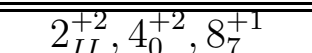 & 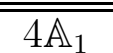 & "18 & "4 \\
\hline & & & & & & $8 \mathbb{A}_{1}$ & 18 & $2_{I I}^{+2}, 4_{7}^{+1}, 8_{7}^{+1}$ \\
\hline & & & & & & $16 \mathbb{A}_{1}$ & 18 & $2_{I I}^{+2}, 4_{6}^{+2}$ \\
\hline 40 & 32 & $\overline{49}$ & $\overline{\bar{Q} Q_{8} * Q_{8}}$ & $\overline{17}$ & $4_{7}^{+5}$ & 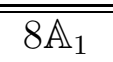 & 18 & $4_{6}^{+4}$ \\
\hline \multirow[t]{3}{*}{ 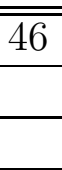 } & 36 & 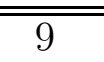 & $3^{2} C_{4}$ & $\overline{18}$ & $2_{6}^{-2}, 3^{+2}, 9^{-1}$ & $\overline{\overline{6 \mathbb{A}_{1}}}$ & $\overline{\overline{19}}$ & $4_{7}^{+1}, 3^{+1}, 9^{-1}$ \\
\hline & & & & & & $9 \mathbb{A}_{1}$ & 19 & $2_{5}^{-3}, 3^{+2}$ \\
\hline & & & & & & $9 \mathbb{A}_{2}$ & 19 & $2_{5}^{-1}, 3^{+2}$ \\
\hline \multirow[t]{3}{*}{48} & $\overline{36}$ & 10 & $\mathfrak{S}_{3,3}$ & 18 & $\bar{C}_{I I I}^{-2}, 3^{+3}, 9^{-1}$ & $\overline{3 \mathbb{A}_{1}}$ & $\overline{19}$ & $2_{5}^{+3}, 3^{-2}, 9^{-1}$ \\
\hline & & & & & & $6 \mathbb{A}_{1}$ & 19 & $4_{1}^{+1}, 3^{+2}, 9^{-1}$ \\
\hline & & & & & & $9 \mathbb{A}_{1}$ & 19 & $2_{7}^{-3}, 3^{+3}$ \\
\hline \multirow[t]{3}{*}{$\overline{49}$} & $\overline{48}$ & $\overline{50}$ & $\overline{2^{4} C_{3}}$ & $\overline{17}$ & $\bar{c}_{I I}^{-4}, 8_{1}^{+1}, 3^{-1}$ & 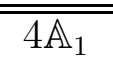 & $\bar{~} 18$ & $\bar{c}_{I I}^{-2}, 4_{I I}^{+2}, 3^{-1}$ \\
\hline & & & & & & $12 \mathbb{A}_{1}$ & 18 & $2_{I I}^{-2}, 4_{2}^{-2}$ \\
\hline & & & & & & $16 \mathbb{A}_{1}$ & 18 & $\frac{-1 I}{2_{I I}^{-4}, 3^{-1}}$ \\
\hline \multirow[t]{5}{*}{$\overline{51}$} & $\overline{48}$ & $\overline{48}$ & $\overline{C_{2} \times \mathfrak{S}_{4}}$ & $\overline{18}$ & 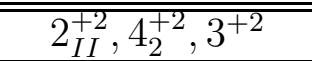 & 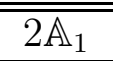 & $\overline{\overline{19}}$ & $\frac{4_{1}^{+3}, 3^{+2}}{}$ \\
\hline & & & & & & $4 \mathbb{A}_{1}$ & 19 & $2_{I I}^{+2}, 8_{1}^{+1}, 3^{+2}$ \\
\hline & & & & & & $6 \mathbb{A}_{1}$ & 19 & $4_{7}^{-3}, 3^{+1}$ \\
\hline & & & & & & $8 \mathbb{A}_{1}$ & 19 & $2_{I I}^{-2}, 4_{5}^{-1}, 3^{+2}$ \\
\hline & & & & & & $12 \mathbb{A}_{1}$ & 19 & $2_{I I}^{-2}, 8_{7}^{+1}, 3^{-1}$ \\
\hline \multirow[t]{5}{*}{$\overline{55}$} & $\overline{60}$ & 5 & $\overline{\mathfrak{A}_{5}}$ & $\overline{18}$ & $\bar{c}_{I I}^{-2}, 3^{+1}, 5^{-2}$ & 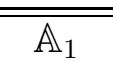 & $\overline{\overline{19}}$ & $\bar{l}_{2}^{-3}, 3^{+1}, 5^{-2}$ \\
\hline & & & & & & $5 \mathbb{A}_{1}$ & 19 & $2_{3}^{+3}, 3^{+1}, 5^{+1}$ \\
\hline & & & & & & $6 \mathbb{A}_{1}$ & 19 & $4_{1}^{+1}, 5^{-2}$ \\
\hline & & & & & & $10 \mathbb{A}_{1}$ & 19 & $4_{7}^{+1}, 3^{+1}, 5^{-1}$ \\
\hline & & & & & & $15 \mathbb{A}_{1}$ & 19 & $2_{5}^{+3}, 5^{-1}$ \\
\hline \multirow[t]{2}{*}{$\overline{56}$} & $\overline{64}$ & $\overline{138}$ & $\overline{\overline{\Gamma_{25} a_{1}}}$ & $\overline{18}$ & $4_{5}^{+3}, 8_{1}^{+1}$ & $8 \mathbb{A}_{1}$ & $\overline{19}$ & $4_{4}^{-2}, 8_{5}^{-1}$ \\
\hline & & & & & & $16 \mathbb{A}_{1}$ & 19 & $4_{5}^{+3}$ \\
\hline \multirow[t]{2}{*}{ "61 } & $\overline{772}$ & $\overline{43}$ & $\overline{\overline{\mathfrak{A}_{4,3}}}$ & $\overline{18}$ & $\overline{4_{I I}^{-2}, 3^{-3}}$ & $3 \mathbb{A}_{1}$ & $\overline{19}$ & $\overline{2}_{5}^{-1}, 4_{I I}^{+2}, 3^{+2}$ \\
\hline & & & & & & $12 \mathbb{A}_{1}$ & 19 & $8_{1}^{+1}, 3^{+2}$ \\
\hline \multirow[t]{4}{*}{$\overline{655}$} & 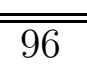 & $\overline{227}$ & $\overline{2^{4} D_{6}}$ & $\overline{18}$ & $2_{I I}^{-2}, 4_{7}^{+1}, 8_{1}^{+1}, 3^{-1}$ & $\overline{4 \mathbb{A}_{1}}$ & $\overline{19}$ & $4_{3}^{-3}, 3^{-1}$ \\
\hline & & & & & & $8 \mathbb{A}_{1}$ & 19 & $2_{I I}^{-2}, 8_{7}^{+1}, 3^{-1}$ \\
\hline & & & & & & $12 \mathbb{A}_{1}$ & 19 & $4_{5}^{+3}$ \\
\hline & & & & & & $16 \mathbb{A}_{1}$ & 19 & $2_{I I}^{+2}, 4_{3}^{-1}, 3^{-1}$ \\
\hline$\overline{75}$ & $\overline{192}$ & $\overline{1023}$ & 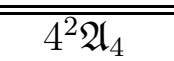 & $\overline{18}$ & 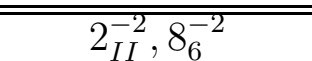 & $\overline{\overline{16 \mathbb{A}_{1}}}$ & $\overline{\overline{19}}$ & 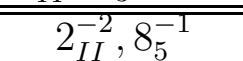 \\
\hline
\end{tabular}


[10] V.V. Nikulin, Finite automorphism groups of Kählerian surfaces of type K3, Uspehi matem. nauk 31 (1976), no. 2, 223-224. (In Russian.)

[11] V.V. Nikulin, Finite automorphism groups of Kähler K3 surfaces, Trudy Mosk. Mat. ob-va V. 38 (1979), 75-137; English transl. in Trans. Moscow Math. Soc. V. 38 (1980), 71-135.

[12] V.V. Nikulin, Integral symmetric bilinear forms and some of their geometric applications, Izv. Akad. Nauk SSSR Ser. Mat. 43 (1979), no. 1, 111-177; English transl. in Math. USSR Izv. 14 (1980), no. 1, 103-167.

[13] V.V. Nikulin, Kählerian K3 surfaces and Niemeier lattices. I, Izvestiya RAN: Ser. Mat. 77 (2013), no. 5, 109-154; English transl.: Izvestya: Mathematics 77 (2013), no. 5, 954-997 (see also arXiv:1109.2879).

[14] V.V. Nikulin, Kählerian K3 surfaces and Niemeier lattices, II, Advanced Studies in Pure Mathematics 69, 2016, Development of Moduli Theory-Kyoto 2013, 421-471 (see also arXiv:1109.2879).

[15] V.V. Nikulin, Degenerations of Kählerian K3 surfaces with finite symplectic automorphism groups, Izvestiya RAN: Ser. Mat. 79 (2015), no. 4, 103-158; English transl.: Izvestya: Mathematics 79 (2015), no. 4, 740-794 (see also arXiv:1403.6061).

[16] V.V. Nikulin, Degenerations of Kählerian K3 surfaces with finite symplectic automorphism groups. II, Izvestiya RAN: Ser. Mat. 80 (2016), no. 2, 81-124; English transl.: Izvestya: Mathematics 80 (2016), no. 2, 359-402 (see also arXiv:1504.00326).

[17] V.V. Nikulin, Degenerations of Kählerian K3 surfaces with finite symplectic automorphism groups. III, Izvestiya RAN: Ser. Mat. 81 (2017), no. 5, 105-149; English transl.: Izvestya: Mathematics 81 (2017), no. 5, 985-1029 (see also arXiv:1608.04373).

[18] V.V. Nikulin Classification of Picard lattices of K3 surfaces, Izvestiya RAN: Ser. Mat. 82 (2018), no. 4, 115-177; English transl.: Izvestya: Mathematics 82 (2018), no. 4, 752-816. (see also arXiv: 1707.05677).

[19] V.V. Nikulin Classification of degenerations and Picard lattices of Kählerian K3 surfaces with the symplectic automorphism group $D_{6}$, Izvestya RAN: Ser. Mat., in publication (see also arXiv:1804.00991). 
[20] V.V. Nikulin Classification of degenerations and Picard lattices of Kählerian K3 surfaces with the symplectic automorphism group $C_{4}$, Proceedings of Steklov Mathematical Institute.

[21] V.V. Nikulin Classification of degenerations and Picard lattices of Kählerian K3 surfaces with small finite symplectic automorphism groups, Preprint 2018. arXiv:1804.00991, 83 pages.

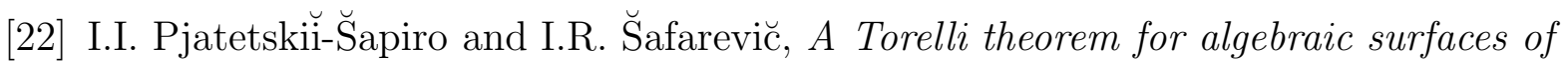
type K3, Izv. AN SSSR. Ser. mat., 35 (1971), no. 3, 530-572; English transl.: Math. USSR Izv. 5 (1971), no. 3, 547-588.

[23] I.R. Shafarevich (ed.), Algebraic Surfaces, Proc. Steklov Math. Inst. 75 (1965),3215 .

[24] Y. Siu, A simple proof of the surjectivity of the period map of K3 surfaces, Manuscripta Math. 35 (1981), no. 3, 311-321.

[25] A. Todorov, Applications of the Kähler-Einstein-Calabi-Yau metric to moduli of K3 surfaces, Invent. math. 61 (1981), no. 3, 251-265.

[26] G. Xiao, Galois covers between K3 surfaces, Ann. Inst. Fourier (Grenoble) 46 (1996), $73-88$.

V.V. Nikulin

Steklov Mathematical Institute,

ul. Gubkina 8, Moscow 117966, GSP-1, Russia;

Department of Mathematical Sciences,

University of Liverpool,

Liverpool, L69 3BX, UK

E-mail: nikulin@mi-ras.ru vnikulin@liv.ac.uk 